\title{
A First Order Hyperbolic Framework For Large Strain Computational Solid Dynamics - Part I: Total Lagrangian Isothermal Elasticity
}

\author{
Javier Bonet ${ }^{1}$, Antonio J. Gil ${ }^{2}$, Chun Hean Lee ${ }^{3}$, Miquel Aguirre, Rogelio \\ Ortigosa \\ Zienkiewicz Centre for Computational Engineering, College of Engineering \\ Swansea University, Singleton Park, SA2 8PP, United Kingdom
}

\begin{abstract}
This paper introduces a new computational framework for the analysis of large strain fast solid dynamics. The paper builds upon previous work published by the authors (Gil et al., 2014) [1], where a first order system of hyperbolic equations is introduced for the simulation of isothermal elastic materials in terms of the linear momentum, the deformation gradient and its Jacobian as unknown variables. In this work, the formulation is further enhanced with four key novelties. First, the use of a new geometric conservation law for the co-factor of the deformation leads to an enhanced mixed formulation, advantageous in those scenarios where the co-factor plays a dominant role. Second, the use of polyconvex strain energy functionals enables the definition of generalised convex entropy functions and associated entropy fluxes for solid dynamics problems. Moreover, the introduction of suitable conjugate entropy variables enables the derivation of a symmetric system of hyperbolic equations, dual of that expressed in terms of conservation variables. Third, the use of a new tensor cross product greatly facilitates the algebraic manipulations of expressions involving the co-factor of the deformation. Fourth, the development of a stabilised Petrov-Galerkin framework is presented for both systems of hyperbolic equations, that is, when expressed in terms of either conservation or entropy variables. As an example, a polyconvex Mooney-
\end{abstract}

\footnotetext{
${ }^{1}$ Corresponding author: j.bonet@swansea.ac.uk

${ }^{2}$ Corresponding author: a.j.gil@swansea.ac.uk

${ }^{3}$ Corresponding author: c.h.lee@swansea.ac.uk
} 
Rivlin material is used and, for completeness, the eigen-structure of the resulting system of equations is studied to guarantee the existence of real wave speeds. Finally, a series of numerical examples is presented in order to assess the robustness and accuracy of the new mixed methodology, benchmarking it against an ample spectrum of alternative numerical strategies, including implicit multi-field Fraeijs de Veubeke-Hu-Washizu variational type approaches and explicit cell and vertex centred Finite Volume schemes.

Keywords: Large strain elasticity, Polyconvex elasticity, Conservation laws, Entropy variables, Stabilised finite elements, Petrov-Galerkin

\section{Introduction}

Traditional displacement-based finite element formulations [2-4] are typically employed when simulating complex engineering large strain transient problems. Linear tetrahedral elements tend to be preferred due to the maturity of the existing unstructured mesh generators [5]. However, this approach presents a number of well-known shortcomings, namely, a reduced order of convergence for strains and stresses [6, 7], poor performance in nearly incompressible bending dominated scenarios [8-10] and numerical instabilities in the form of shear locking, volumetric locking and spurious hydrostatic pressure fluctuations [11-13]. In addition, displacement-based methods used in conjunction with Newmark-type time integrators [14] have a tendency to introduce high frequency noise and accuracy is degraded once numerical artificial damping is employed [15-19].

Some of these numerical difficulties can be addressed with the use of high order approaches [20-22]. However, the increase in the number of integration points can drastically reduce the computational efficiency of these schemes in comparison with low order approaches, especially when complex constitutive laws are of interest (e.g. visco-elasticity, visco-plasticity).

Significant work has been undertaken to develop effective linear tetrahedral formulations for solid mechanics. Multi-field Fraeijs de Veubeke-HuWashizu (FdVHW) type variational principles [23] are among them, where independent kinematic descriptions are used for the volumetric and deviatoric contributions of the deformation, such as the conventional mean dilatation method [24]. Unfortunately, the latter approach cannot be employed with linear tetrahedral elements and it is typical to resort to some form of projection to reduce the number of volumetric constraints [24-33]. 
The very first family of nodally integrated tetrahedral elements was formulated by Bonet and Burton [34]. The resulting methodology performed extremely well in nearly incompressible impact problems but was found to behave poorly in bending dominated scenarios. Since then, numerous attempts have been reported at improving the robustness of the formulation $[9,10,13,35]$. However, resulting approaches suffer from artificial mechanisms similar to hourglassing unless some form of stabilisation is used [13, 3640].

Reference [41] presented a stabilised Petrov-Galerkin (PG) mixed formulation using Galerkin Least Squares stabilisation for the analysis of the Stokes problem, with equal order of interpolation for velocity and pressure. The formulation circumvents the Ladyzenskaya-Babuska-Brezzi (LBB) condition ensuring both numerical stability and optimal convergence. This approach has been extended to linear tetrahedral elements in the context of elastostatics [13, 42-46]. However, the development of an effective linear tetrahedral formulation in the range of large strain dynamics still remains an open issue $[1,47,48]$.

To overcome all the shortcomings mentioned above, a mixed methodology was introduced where the linear momentum $\boldsymbol{p}$ and the deformation gradient $\boldsymbol{F}$ were treated as primary variables of a new mixed approach in the form of a system of conservation laws [8, 49-52]. The two-field formulation was recently augmented by incorporating a new conservation law for the Jacobian of the deformation $J[53,54]$ to effectively solve bending dominated nearly incompressible deformations $[1,55,56]$. Moreover, the formulation was also extended to account for truly incompressible materials utilising a fractional step approach [57-60] to circumvent the volumetric wave speed constraint.

One of the key novelties in this paper is the new use of a tensor cross product operation, along with its associated algebra [46], presented for the first time in Reference [61], page 76, which greatly simplifies the notation and enables the introduction of a new conservation equation for the area map tensor $\boldsymbol{H}$ (co-factor of the deformation gradient). This new geometrical conservation law is then solved along with those already described by the authors in previous publications [1, 8, 49, 50, 52], yielding a more general computational framework.

The enhanced mixed methodology is formulated in the form of a system of first order conservation laws [62,63], where the linear momentum $\boldsymbol{p}$ conservation equation is now supplemented with three geometric conservation laws for the minors of the deformation tensor (i.e. one for the deformation 
gradient $\boldsymbol{F}$, one for the area map $\boldsymbol{H}$ and one for the Jacobian of the deformation $J$ ). Crucially, and in line with the pioneering work of Hughes et al. [64] in the context of Computational Fluid Dynamics, the consideration of polyconvex energy functionals in nonlinear elasticity [65-71] enables the definition of a generalised convex entropy function (and associated entropy flux).

This innovative idea facilitates the transformation of the system of conservation laws into a symmetric set of hyperbolic equations when expressed in terms of the entropy conjugates of the conservation variables. This guarantees the existence of real wave speeds, and hence material stability, due to the fact that the Hessian operator is positive definite [45, 46]. Moreover, the formulation in terms of entropy variables can lead to alternative forms of stabilisation as presented in [72].

This paper is the first one in a series aiming to further explore the use of a first order hyperbolic system of conservation laws in the context of large strain fast dynamics. The outline of the present paper is as follows. In section 2, we start by introducing a new tensor cross product which greatly simplifies the definition of the area map tensor (co-factor of the deformation). A full set of total Lagrangian conservation laws, in the form of a system of first order hyperbolic equations, as well as the definition of a polyconvex energy functional are presented in section 3. In section 4, the consideration of polyconvexity enables the symmetrisation of the hyperbolic system with an appropriate change of variables, from the conservation variables to its entropy conjugate counterparts. As an example, the eigenvalue structure of a Mooney-Rivlin model is also presented. In section 5, a new variationally consistent Petrov-Galerkin (PG) stabilisation methodology (a particular case of Variational Multi-Scale method [73]) for both conservation and entropy variables is derived. This section ends with the description of the Runge-Kutta time integrator employed and the PG entropy-based stabilisation particularised for one and two dimensional scenarios. In section 6 , a series of numerical examples are presented to assess the robustness of the mixed formulations and to draw some comparisons against previous results published by the authors $[1,8,46,50,52]$. Finally, section 7 presents some concluding remarks and current directions of research. 


\section{Definitions and notation}

\subsection{Motion and deformation}

Consider the three dimensional deformation of an elastic body moving from its initial configuration occupying a volume $V$, of boundary $\partial V$, into a current configuration at time $t$ occupying volume $v$, see Figure 1 . The standard notation and definitions for the deformation gradient $\boldsymbol{F}$ and its determinant $J$ are used:

$$
\boldsymbol{F}=\frac{\partial \boldsymbol{x}}{\partial \boldsymbol{X}}=\nabla_{0} \boldsymbol{x} ; \quad J=\operatorname{det} \boldsymbol{F}=\frac{d v}{d V}
$$

where $\boldsymbol{x}$ represents the current position of a particle originally at $\boldsymbol{X}$ and $\boldsymbol{\nabla}_{0}$ denotes the gradient with respect to material coordinates. Time derivatives or virtual variations of $\boldsymbol{x}$ will be denoted $\boldsymbol{v}$ and $\delta \boldsymbol{v}$, respectively, and will satisfy appropriate boundary conditions in $\partial_{u} V$. Additionally, the body is under the action of certain body forces per unit undeformed volume $\boldsymbol{f}_{0}$ and traction per unit undeformed area $\boldsymbol{t}_{0}$ in $\partial_{t} V$, where $\partial_{t} V \cup \partial_{u} V=\partial V$ and $\partial_{t} V \cap \partial_{u} V=\emptyset$.

The element of area vector is mapped from initial $d \boldsymbol{A}$ to final $d \boldsymbol{a}$ configuration by means of the two-point tensor $\boldsymbol{H}$, which is related to the deformation gradient via Nanson's rule [11, 74]:

$$
d \boldsymbol{a}=\boldsymbol{H} d \boldsymbol{A} ; \quad \boldsymbol{H}=J \boldsymbol{F}^{-T} .
$$

The components of this tensor are the order 2 minors of the deformation gradient and it is often referred to as the co-factor or adjoint tensor. This tensor and its time derivative will feature heavily in the formulation that follows as it is a key variable for polyconvex elastic models. Its evaluation and, more importantly, the evaluation of its time derivative using equation $(2)_{2}$ is not ideal, and a more convenient formula can be derived for three dimensional applications. This relies on the use of a tensor cross product operation, presented for the first time in Reference [61], page 76, but included in Appendix A for completeness.

\subsection{Alternative expressions for the area and volume maps}

Using the property in Appendix A, equation (A.17), it is now possible to express the area map tensor $\boldsymbol{H}$ as:

$$
\boldsymbol{H}=\frac{1}{2} \boldsymbol{F} \times \boldsymbol{F}
$$




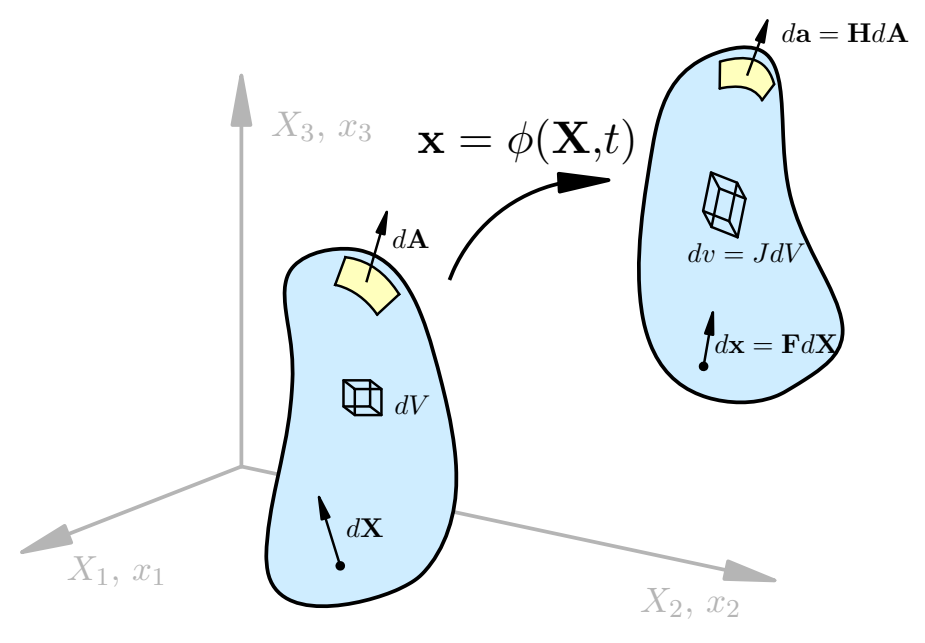

Figure 1: Deformation mapping of a continuum and associated kinematics magnitudes: $\boldsymbol{F}, \boldsymbol{H}, J$.

It is also possible to derive alternative expressions for both $\boldsymbol{H}$ and $J$. For instance, combining equation (3) and equation (1) and noting that the derivatives of $\boldsymbol{F}$ are second derivatives of $\boldsymbol{x}$ and, therefore symmetric, gives, after simple use of the product rule:

$$
\boldsymbol{H}=\frac{1}{2} \operatorname{CURL}(\boldsymbol{x} \times \boldsymbol{F})
$$

where the material CURL of a second order two-point tensor is defined in the usual fashion by:

$$
(\operatorname{CURL} \boldsymbol{A})_{i I}=\mathcal{E}_{I J K} \frac{\partial A_{i K}}{\partial X_{J}} .
$$

It is clear from equation (4) that the material divergence of $\boldsymbol{H}$ vanishes, as does the material CURL of $\boldsymbol{F}$, that is:

$$
\text { DIVH }=0 ; \quad \text { CURLF }=0
$$

where the material divergence is defined by the contraction:

$$
(\operatorname{DIV} \boldsymbol{A})_{i}=\frac{\partial A_{i I}}{\partial X_{I}}
$$


Combining equations (1) $)_{1}$ and (3) and alternative equation for $J$ emerges as:

$$
J=\frac{1}{3} \boldsymbol{H}: \nabla_{0} \boldsymbol{x}=\frac{1}{3} \operatorname{DIV}\left(\boldsymbol{H}^{T} \boldsymbol{x}\right) .
$$

In the context of the conservation laws that will be developed in the following sections, equations (6) represent two sets of involutions [75, 76] or constraints that need to be satisfied by the conservation variables.

\section{Total Lagrangian conservation laws}

\subsection{General remarks}

The aim of this section is to express the equations of large strain solid dynamics in the form of a set of global conservation laws for a set of conservation variables $\mathcal{U}=\left[\mathcal{U}_{\alpha}\right]$ with fluxes $\mathcal{F}=\left[\mathcal{F}_{\alpha I}\right]$ and source term $\mathcal{S}=\left[\mathcal{S}_{\alpha}\right]$, where $\alpha=1, \ldots, n$ represents the set of unknowns and $I=1,2,3$ the reference coordinates. Global conservation laws are generally expressed as:

$$
\frac{d}{d t} \int_{V} \mathcal{U} d V+\int_{\partial V} \mathcal{F} d \boldsymbol{A}=\int_{V} \mathcal{S} d V
$$

Note that $V$ in the above integrals represents an arbitrary Lagrangian volume fixed in the initial reference configuration. This need not coincide with the actual entire volume of the moving body. For smooth functions, this integral expression is equivalent to the set of first order differential equations:

$$
\frac{\partial \mathcal{U}}{\partial t}+\operatorname{DIV} \mathcal{F}=\mathcal{S}
$$

In addition, for discontinuous solutions the integral conservation law also leads to the following jump conditions across a discontinuity surface with normal $\boldsymbol{N}$ moving with speed $U[50,77,78]$ :

$$
U \llbracket \mathcal{U} \rrbracket=\llbracket \mathcal{F} \rrbracket N
$$

where the notation $\llbracket \phi \rrbracket=\left(\phi_{+}-\phi_{-}\right)$is used to denote the jump of a variable across a moving discontinuity surface. It is often convenient to express equation (10) in the quasi-linear form:

$$
\frac{\partial \mathcal{U}}{\partial t}+\mathcal{A}_{I} \frac{\partial \mathcal{U}}{\partial X_{I}}=\mathcal{S} ; \quad \mathcal{A}_{I}=\left[\mathcal{A}_{\alpha \beta}\right]_{I}=\frac{\partial \mathcal{F}_{I}}{\partial \mathcal{U}},
$$


where $\mathcal{F}_{I}$ denotes the columns of the flux matrix and, in general, the square $n \times n$ matrices $\mathcal{A}_{I}$ (with $\left.\alpha, \beta=1, \ldots, n\right)$ will not be symmetric.

Alternatively, the flux matrix $\mathcal{F}$ defined in (10) can be expressed as

$$
\mathcal{F}=\mathcal{F}_{I} \otimes \boldsymbol{E}_{I} ; \quad \boldsymbol{E}_{1}=\left[\begin{array}{l}
1 \\
0 \\
0
\end{array}\right] ; \quad \boldsymbol{E}_{2}=\left[\begin{array}{l}
0 \\
1 \\
0
\end{array}\right] ; \quad \boldsymbol{E}_{3}=\left[\begin{array}{l}
0 \\
0 \\
1
\end{array}\right]
$$

\subsection{Conservation of mass and momentum}

For Lagrangian formulations conservation of mass is simply stated by

$$
\frac{d}{d t} \int_{V} \rho_{0} d V=0
$$

This simply implies that the initial density of the material $\rho_{0}$ is constant and therefore does not need to be considered as part of the vector of problem unknowns $\mathcal{U}$.

Global conservation of linear momentum $\boldsymbol{p}=\rho_{0} \boldsymbol{v}$ is established for any arbitrary Lagrangian volume $V$ by:

$$
\frac{d}{d t} \int_{V} \boldsymbol{p} d V=\int_{\partial V} \boldsymbol{t}_{0} d A+\int_{V} \boldsymbol{f}_{0} d V
$$

where $\boldsymbol{f}_{0}$ represents a body force per unit undeformed volume and $\boldsymbol{t}_{0}$ the traction vector associated with the initial normal $\boldsymbol{N}$. Assuming the existence of a first Piola-Kirchhoff stress tensor $\boldsymbol{P}$ such that the traction vector associated with the initial area with normal $\boldsymbol{N}$ is $\boldsymbol{t}_{0}=\boldsymbol{P} \boldsymbol{N}$, the equivalent local equilibrium equation and jump condition can be written as:

$$
\frac{\partial \boldsymbol{p}}{\partial t}-\operatorname{DIVP}=\boldsymbol{f}_{0} ; \quad U \llbracket \boldsymbol{p} \rrbracket=-\llbracket \boldsymbol{P} \rrbracket \boldsymbol{N} .
$$

In general, the first Piola-Kirchhoff stresses are a function of the deformation gradient $\boldsymbol{F}$, temperature and a set of state variables that describe, for instance, the accumulated plastic deformation. This paper considers only the case of isothermal elasticity, although the extension to elastoplasticity or viscoelasticity is straightforward $[8,50,52]$. Under such conditions, the Piola-Kirchhoff stress is derived from an elastic potential function $\Psi(\boldsymbol{F})$ as:

$$
\boldsymbol{P}(\boldsymbol{F})=\frac{\partial \Psi}{\partial \boldsymbol{F}}
$$


In general, the strain energy functional $\Psi(\boldsymbol{F})$ is not convex in $\boldsymbol{F}$ and, therefore, the resulting stress-strain relationship is not one-to-one. This implies that given a stress tensor $\boldsymbol{P}$ it is not possible to determine the corresponding deformation gradient $\boldsymbol{F}$ and, therefore, it will not be possible to use this stress tensor as a suitable conjugate entropy variable to the deformation gradient. In order to overcome this, it is necessary to introduce constitutive laws defined by means of a polyconvex elastic strain energy.

\subsection{Constitutive laws: Polyconvex elasticity}

Polyconvexity $[65-68,79]$ is now well accepted as a fundamental mathematical requirement that must be satisfied by admissible strain energy functions used to describe elastic materials in large strain regime. Essentially, the strain energy $\Psi$ per unit undeformed volume must be a function of the deformation gradient $\boldsymbol{F}$ via a convex multi-valued function $W[62]$ as:

$$
\Psi(\boldsymbol{F})=W(\boldsymbol{F}, \boldsymbol{H}, J)
$$

where $W$ is convex with respect to its 19 variables, namely, $J$ and the $3 \times 3$ components of $\boldsymbol{F}$ and $\boldsymbol{H}$. Moreover, invariance with respect to rotations in the material configuration implies that $W$ must be independent of the rotational components of $\boldsymbol{F}$ and $\boldsymbol{H}$. This is typically achieved by ensuring that $W$ depends on $\boldsymbol{F}$ and $\boldsymbol{H}$ via the symmetric tensors $\boldsymbol{C}=\boldsymbol{F}^{T} \boldsymbol{F}$ and $\boldsymbol{G}=\boldsymbol{H}^{T} \boldsymbol{H}$, respectively. In fact, for isotropic materials, this dependency can be further simplified through the use of invariants and the observation that:

$$
I_{C}=\boldsymbol{F}: \boldsymbol{F} ; \quad I I_{C}=\boldsymbol{H}: \boldsymbol{H} ; \quad I I I_{C}=J^{2} .
$$

For example, a general compressible Mooney-Rivlin material can be described by an energy function of the type:

$$
W_{M R}=\alpha \boldsymbol{F}: \boldsymbol{F}+\beta \boldsymbol{H}: \boldsymbol{H}+f(J)
$$

where $\alpha$ and $\beta$ are positive material parameters and $f$ denotes a convex function of $J$. It is therefore clear that $W_{M R}$ is convex with respect to all of its 19 variables. The case $\beta=0$ gives the simpler compressible Neo-Hookean model $W_{N H}$, which is convex in $F$ and $J$ alone without the need to introduce $\boldsymbol{H}$ as a separate independent variable.

The condition of vanishing energy at the initial reference configuration can be established by ensuring that $f(1)=-3(\alpha+\beta)$ or by adding an 
appropriate constant to $W_{M R}$. Doing this, however, has no practical effect on the resulting formulation as this will be driven by derivatives of the strain energy. However, appropriate values for $\alpha$ and $\beta$ and suitable functions $f(J)$ must be such that, at the initial configuration, the stress vanishes and the usual linear elasticity tensor in terms of the Lamé coefficients $\lambda$ and $\mu$ is recovered. This leads to the following set of conditions [46]:

$$
\begin{aligned}
f^{\prime}(1) & =-2(\alpha+2 \beta) \\
f^{\prime \prime}(1) & =\lambda+2 \alpha \\
\mu & =2(\alpha+\beta) .
\end{aligned}
$$

A commonly used convex expression for $f(J)$ that satisfies these requirements is:

$$
f(J)=-4 \beta J-2 \alpha \ln J+\frac{\lambda}{2}(J-1)^{2} .
$$

In particular, for the Neo-Hookean case $\alpha$ and $\beta$ become $\frac{\mu}{2}$ and 0 , respectively.

The three strain measures $\boldsymbol{F}, \boldsymbol{H}$ and $J$ have work conjugate stresses $\boldsymbol{\Sigma}_{\boldsymbol{F}}$, $\boldsymbol{\Sigma}_{\boldsymbol{H}}$ and $\Sigma_{J}$ defined by $[45,46,62,63]$ :

$$
\boldsymbol{\Sigma}_{\boldsymbol{F}}(\boldsymbol{F}, \boldsymbol{H}, J)=\frac{\partial W}{\partial \boldsymbol{F}} ; \quad \boldsymbol{\Sigma}_{\boldsymbol{H}}(\boldsymbol{F}, \boldsymbol{H}, J)=\frac{\partial W}{\partial \boldsymbol{H}} ; \quad \Sigma_{J}(\boldsymbol{F}, \boldsymbol{H}, J)=\frac{\partial W}{\partial J} .
$$

For instance, for the case of a Mooney-Rivlin material, with the aid of equation (20), these stresses become:

$$
\boldsymbol{\Sigma}_{\boldsymbol{F}}=2 \alpha \boldsymbol{F} ; \quad \boldsymbol{\Sigma}_{\boldsymbol{H}}=2 \beta \boldsymbol{H} ; \quad \Sigma_{J}=f^{\prime}(J) .
$$

By comparing the time derivative of the strain energy defined in terms of $\Psi$ (18) to the time derivative of $W$ and using the properties of the tensor cross product shown in Appendix $\mathrm{A}$, it is possible to relate the conjugate stresses defined in equation (23) to the standard first Piola-Kirchhoff stress tensor as:

$$
\begin{aligned}
\boldsymbol{P}: \dot{\boldsymbol{F}} & =\dot{\Psi}(\boldsymbol{F}) \\
& =\dot{W}(\boldsymbol{F}, \boldsymbol{H}, J) \\
& =\boldsymbol{\Sigma}_{\boldsymbol{F}}: \dot{\boldsymbol{F}}+\boldsymbol{\Sigma}_{\boldsymbol{H}}: \dot{\boldsymbol{H}}+\Sigma_{J} \dot{J} \\
& =\boldsymbol{\Sigma}_{\boldsymbol{F}}: \dot{\boldsymbol{F}}+\boldsymbol{\Sigma}_{\boldsymbol{H}}:(\boldsymbol{F} \times \dot{\boldsymbol{F}})+\Sigma_{J} \boldsymbol{H}: \dot{\boldsymbol{F}} \\
& =\left(\boldsymbol{\Sigma}_{\boldsymbol{F}}+\boldsymbol{\Sigma}_{\boldsymbol{H}} \times \boldsymbol{F}+\Sigma_{J} \boldsymbol{H}\right): \dot{\boldsymbol{F}} .
\end{aligned}
$$


This leads to the following relationship [46]:

$$
\boldsymbol{P}=\Sigma_{\boldsymbol{F}}+\boldsymbol{\Sigma}_{\boldsymbol{H}} \times \boldsymbol{F}+\Sigma_{J} \boldsymbol{H}
$$

For instance, for the simple compressible Mooney-Rivlin material defined above, this expression becomes:

$$
\boldsymbol{P}=2 \alpha \boldsymbol{F}+2 \beta(\boldsymbol{H} \times \boldsymbol{F})+f^{\prime}(J) \boldsymbol{H} .
$$

The convexity of the function $W(\boldsymbol{F}, \boldsymbol{H}, J)$ with respect to its variables ensures that the relationships between $\{\boldsymbol{F}, \boldsymbol{H}, J\}$ and $\left\{\boldsymbol{\Sigma}_{\boldsymbol{F}}, \boldsymbol{\Sigma}_{\boldsymbol{H}}, \Sigma_{J}\right\}$ is oneto-one and invertible. It is therefore possible to define a complementary energy function by means of the Legendre transform [80] as:

$$
\Upsilon\left(\boldsymbol{\Sigma}_{\boldsymbol{F}}, \boldsymbol{\Sigma}_{\boldsymbol{H}}, \Sigma_{J}\right)=\max _{\{\boldsymbol{F}, \boldsymbol{H}, J\}}\left\{\boldsymbol{\Sigma}_{\boldsymbol{F}}: \boldsymbol{F}+\boldsymbol{\Sigma}_{\boldsymbol{H}}: \boldsymbol{H}+\Sigma_{J} J-W(\boldsymbol{F}, \boldsymbol{H}, J)\right\}
$$

which defines the reverse constitutive relationship as:

$\boldsymbol{F}\left(\boldsymbol{\Sigma}_{\boldsymbol{F}}, \boldsymbol{\Sigma}_{\boldsymbol{H}}, \Sigma_{J}\right)=\frac{\partial \Upsilon}{\partial \boldsymbol{\Sigma}_{\boldsymbol{F}}} ; \quad \boldsymbol{H}\left(\boldsymbol{\Sigma}_{\boldsymbol{F}}, \boldsymbol{\Sigma}_{\boldsymbol{H}}, \Sigma_{J}\right)=\frac{\partial \Upsilon}{\partial \boldsymbol{\Sigma}_{\boldsymbol{H}}} ; \quad J\left(\boldsymbol{\Sigma}_{\boldsymbol{F}}, \boldsymbol{\Sigma}_{\boldsymbol{H}}, \Sigma_{J}\right)=\frac{\partial \Upsilon}{\partial \Sigma_{J}}$

Often it is necessary to derive expressions for the symmetric Cauchy and Kirchhoff stresses (i.e. $\boldsymbol{\sigma}$ and $\boldsymbol{\tau}$ respectively) as these stresses are needed to express plasticity models [74] or simply to display solution results. Such expressions can be easily derived from the standard relationship between these tensors $[11,74,81]$ :

$$
J \boldsymbol{\sigma}=\boldsymbol{\tau}=\boldsymbol{P} \boldsymbol{F}^{T}
$$

Substituting the definition of Piola $\boldsymbol{P}(26)$ into (30) and the use of $\boldsymbol{H} \boldsymbol{F}^{T}=J \boldsymbol{I}$ gives

$$
J \boldsymbol{\sigma}=\boldsymbol{\tau}=\boldsymbol{\Sigma}_{\boldsymbol{F}} \boldsymbol{F}^{T}+\left(\boldsymbol{\Sigma}_{\boldsymbol{H}} \times \boldsymbol{F}\right) \boldsymbol{F}^{T}+J \Sigma_{J} \boldsymbol{I}
$$

The second term on the right hand side of the expression above can be transformed with the aid of (A.18) by taking $\boldsymbol{A}=J^{-1} \boldsymbol{\Sigma}_{\boldsymbol{H}} \boldsymbol{H}^{T}, \boldsymbol{B}=\boldsymbol{I}$ and $\boldsymbol{C}=\boldsymbol{F}$ (see property $A .17$ ) to give:

$$
\left(\boldsymbol{\Sigma}_{\boldsymbol{H}} \times \boldsymbol{F}\right) \boldsymbol{F}^{T}=\left(\boldsymbol{\Sigma}_{\boldsymbol{H}} \boldsymbol{H}^{T}\right) \times \boldsymbol{I}
$$


Thus the expression for the Kirchhoff stresses as:

$$
J \boldsymbol{\sigma}=\boldsymbol{\tau}=\boldsymbol{\Sigma}_{\boldsymbol{F}} \boldsymbol{F}^{T}+\left(\boldsymbol{\Sigma}_{\boldsymbol{H}} \boldsymbol{H}^{T}\right) \times \boldsymbol{I}+J \Sigma_{J} \boldsymbol{I}
$$

Alternatively, introducing the notation:

$$
\boldsymbol{\tau}_{\boldsymbol{F}}=\boldsymbol{\Sigma}_{\boldsymbol{F}} \boldsymbol{F}^{T} ; \quad \boldsymbol{\tau}_{\boldsymbol{H}}=\boldsymbol{\Sigma}_{\boldsymbol{H}} \boldsymbol{H}^{T} ; \quad \tau_{J}=J \Sigma_{J}
$$

gives,

$$
J \boldsymbol{\sigma}=\boldsymbol{\tau}=\boldsymbol{\tau}_{\boldsymbol{F}}+\boldsymbol{\tau}_{\boldsymbol{H}} \times \boldsymbol{I}+\tau_{J} \boldsymbol{I} .
$$

\subsection{Conservation of deformation gradient}

Traditional displacement based solid mechanics formulations evaluate the deformation gradient $\boldsymbol{F}$ by taking the gradient of the current geometry using equation $(1)_{1}[10,34,37]$. However, this approach leads to a formulation that is second order in time for $\boldsymbol{x}$ in which strains and stresses converge at a rate one order lower than the geometry or displacement. The formulation proposed here is based on deriving independent first order conservation laws for the strain variables $[49-51,55]$. In order to evaluate global and local conservation laws for $\boldsymbol{F}$, note first that integrating equation (1) ${ }_{1}$ over an arbitrary reference volume and using the Gauss theorem gives:

$$
\int_{V} \boldsymbol{F} d V=\int_{V} \boldsymbol{\nabla}_{0} \boldsymbol{x} d V=\int_{\partial V} \boldsymbol{x} \otimes d \boldsymbol{A} .
$$

Differentiating with respect to time gives a global conservation law for the deformation gradient as:

$$
\frac{d}{d t} \int_{V} \boldsymbol{F} d V=\int_{\partial V} \boldsymbol{v} \otimes d \boldsymbol{A} .
$$

The corresponding local differential equation and jump condition are:

$$
\frac{\partial \boldsymbol{F}}{\partial t}-\mathrm{DIV}\left(\frac{1}{\rho_{0}} \boldsymbol{p} \otimes \boldsymbol{I}\right)=\mathbf{0} ; \quad U \llbracket \boldsymbol{F} \rrbracket=-\llbracket \frac{1}{\rho_{0}} \boldsymbol{p} \rrbracket \otimes \boldsymbol{N} .
$$




\subsection{Conservation of area map}

A similar conservation law can be derived for the area map tensor $\boldsymbol{H}$. For this purpose, note first that integrating equation (4) over a reference volume gives:

$$
\int_{V} \boldsymbol{H} d V=-\int_{\partial V} \frac{1}{2} \boldsymbol{x} \times \boldsymbol{F} \times \boldsymbol{N} d A .
$$

Differentiating this expression with respect to time and noting that $\boldsymbol{F}=$ $\boldsymbol{\nabla}_{0} \boldsymbol{x}$ gives, after some algebraic manipulation, a global conservation law for the area map as:

$$
\frac{d}{d t} \int_{V} \boldsymbol{H} d V=\int_{\partial V} \boldsymbol{F} \times(\boldsymbol{v} \otimes d \boldsymbol{A}) .
$$

This expression is equivalent to a local conservation law for $\boldsymbol{H}$ and jump condition given by:

$$
\frac{\partial \boldsymbol{H}}{\partial t}-\operatorname{CURL}\left(\frac{1}{\rho_{0}} \boldsymbol{p} \times \boldsymbol{F}\right)=\mathbf{0} ; \quad U \llbracket \boldsymbol{H} \rrbracket=-\boldsymbol{F} \times\left(\llbracket \frac{1}{\rho_{0}} \boldsymbol{p} \rrbracket \otimes \boldsymbol{N}\right) .
$$

\subsection{Conservation of volume map}

A final conservation law for the volume ratio $J$ can be conveniently derived by starting from the following "Updated Lagrangian" expression for the rate of change of a moving volume $v$ initially at $V[73,82,83]$ :

$$
\frac{d}{d t} \int_{v(V)} d v=\int_{\partial v(V)} \boldsymbol{v} \cdot d \boldsymbol{a} .
$$

The total Lagrangian equivalent of the above equation is easily obtained from the volume and area maps as:

$$
\frac{d}{d t} \int_{V} J d V=\int_{\partial V} \boldsymbol{H}:(\boldsymbol{v} \otimes d \boldsymbol{A}) .
$$

The following local conservation law and jump condition are derived from the above global law:

$$
\frac{\partial J}{\partial t}-\mathbf{D I V}\left(\frac{1}{\rho_{0}} \boldsymbol{H}^{T} \boldsymbol{p}\right)=0 ; \quad U \llbracket J \rrbracket=-\boldsymbol{H}:\left(\llbracket \frac{1}{\rho_{0}} \boldsymbol{p} \rrbracket \otimes \boldsymbol{N}\right) .
$$




\subsection{Combined equations}

Combining the results of the various sections above, a full set of first order conservation laws (10) can be established with vector of variables $\mathcal{U}$, flux vector $\mathcal{F}_{I}$ and source terms $\mathcal{S}$ given by:

$$
\mathcal{U}=\left[\begin{array}{c}
\boldsymbol{p} \\
\boldsymbol{F} \\
\boldsymbol{H} \\
J
\end{array}\right] ; \quad \mathcal{F}_{I}=-\left[\begin{array}{c}
\boldsymbol{P} \boldsymbol{E}_{I} \\
\frac{1}{\rho_{0}} \boldsymbol{p} \otimes \boldsymbol{E}_{I} \\
\boldsymbol{F} \times\left(\frac{1}{\rho_{0}} \boldsymbol{p} \otimes \boldsymbol{E}_{I}\right) \\
\boldsymbol{H}:\left(\frac{1}{\rho_{0}} \boldsymbol{p} \otimes \boldsymbol{E}_{I}\right)
\end{array}\right] ; \quad \mathcal{S}=\left[\begin{array}{c}
\boldsymbol{f}_{0} \\
\mathbf{0} \\
\mathbf{0} \\
0
\end{array}\right]
$$

Notice that since $\boldsymbol{F}$ and $\boldsymbol{H}$ are $3 \times 3$ tensors, the above notation must be interpreted so that their components are incorporated into the $22 \times 1$ column vector of unknowns $\mathcal{U}$ by placing their columns vertically underneath each other. A corresponding arrangement is used for the $22 \times 3$ flux matrix $\mathcal{F}$ and $22 \times 1$ source term vector $\mathcal{S}$. In order to avoid confusion, this combined matrix notation will be avoided if possible in favour of using the individual conservation laws for each component.

Inevitably, there is a significant amount of redundancy in the above set of equations. This redundancy manifests itself via the involutions defined by equation (6) or through expressions of the type $\boldsymbol{F}^{T} \boldsymbol{H}=J \boldsymbol{I}$. It is important that the computational technique used to discretise the above system takes into account these sets of involutions [51, 52]. References [8] and [1] have considered the reduced systems where only $\boldsymbol{F}$ or $\boldsymbol{F}$ and $J$ are included. These have proved robust in most situations. In general, however, only the full system is capable of being symmetrised when expressed in terms of conjugate stress variables. The only exception to this statement takes place in the case of material models such as the compressible Neo-Hookean for which the strain energy can be expressed as a convex function of $\boldsymbol{F}$ and $J$ alone. For such cases the combined system becomes:

$$
\mathcal{U}=\left[\begin{array}{c}
\boldsymbol{p} \\
\boldsymbol{F} \\
J
\end{array}\right] ; \quad \mathcal{F}_{I}=-\left[\begin{array}{c}
\left(\boldsymbol{\Sigma}_{\boldsymbol{F}}+\Sigma_{J} \boldsymbol{H}_{\boldsymbol{F}}\right) \boldsymbol{E}_{I} \\
\frac{1}{\rho_{0}} \boldsymbol{p} \otimes \boldsymbol{E}_{I} \\
\boldsymbol{H}:\left(\frac{1}{\rho_{0}} \boldsymbol{p} \otimes \boldsymbol{E}_{I}\right)
\end{array}\right] ; \quad \mathcal{S}=\left[\begin{array}{c}
\boldsymbol{f}_{0} \\
\mathbf{0} \\
0
\end{array}\right]
$$


In this expression the notation $\boldsymbol{H}_{\boldsymbol{F}}=(\operatorname{det} \boldsymbol{F}) \boldsymbol{F}^{-T}$ indicates that the area map tensor is evaluated directly from the deformation gradient $\boldsymbol{F}$ rather than as an independent variable from its own conservation law.

\section{Symmetrisation of the elastodynamics equations}

\subsection{General remarks}

Quasi-linear first order hyperbolic systems can be symmetrised with an appropriate change of variables [64], if there exists a convex generalised entropy function $S(\mathcal{U})$ and corresponding entropy flux $\boldsymbol{\Lambda}(\mathcal{U})$ such that in the absence of source terms and for all admissible solutions,

$$
\frac{\partial S}{\partial t}+\operatorname{DIV} \Lambda \geq 0
$$

The above inequality becomes an equality for smooth solutions in the absence of dissipative effects such as viscosity or heat flow, which is the case here. It is now possible to define a new set of conjugate entropy variables $\mathcal{V}=\frac{\partial S}{\partial \mathcal{U}}$ for which the conservation laws (12) become:

$$
\mathcal{A}_{0} \frac{\partial \mathcal{V}}{\partial t}+\tilde{\mathcal{A}}_{I} \frac{\partial \mathcal{V}}{\partial X_{I}}=\mathcal{S}
$$

The symmetric system matrices $\mathcal{A}_{0}$ and $\tilde{\mathcal{A}}_{I}$ are:

$$
\mathcal{A}_{0}=\frac{\partial \mathcal{U}}{\partial \mathcal{V}}=\left[\frac{\partial^{2} S}{\partial \mathcal{U} \partial \mathcal{U}}\right]^{-1} ; \quad \tilde{\mathcal{A}}_{I}=\mathcal{A}_{I} \mathcal{A}_{0}=\tilde{\mathcal{A}}_{I}^{T}
$$

and the entropy flux $\Lambda_{I}$ satisfies:

$$
\frac{\partial \Lambda_{I}}{\partial \mathcal{U}}=\mathcal{V}^{T} \mathcal{A}_{I}
$$

Note also that pre-multiplying the expression (48) by $\mathcal{A}_{0}^{-1}$ and taking

advantage of the symmetry of $\tilde{\mathcal{A}}_{I}$ gives a conjugate set of equations for the entropy variables as:

$$
\frac{\partial \mathcal{V}}{\partial t}+\mathcal{A}_{I}^{T} \frac{\partial \mathcal{V}}{\partial X_{I}}=\mathcal{A}_{0}^{-1} \mathcal{S}
$$


Moreover, as presented in [72], given the symmetry and positive definiteness of $\mathcal{A}_{0}$ (i.e. $S$ is a convex entropy function), it is possible to define the following (non-unique) multiplicative decomposition $\mathcal{A}_{0}=\mathcal{L} \mathcal{L}^{T}$, which after substitution into (48) renders

$$
\mathcal{L}^{T} \frac{\partial \mathcal{V}}{\partial t}+\hat{\mathcal{A}}_{I} \mathcal{L}^{T} \frac{\partial \mathcal{V}}{\partial X_{I}}=\mathcal{L}^{-1} \mathcal{S} ; \quad \hat{\mathcal{A}}_{I}=\mathcal{L}^{-1} \tilde{\mathcal{A}}_{I} \mathcal{L}^{-T}=\hat{\mathcal{A}}_{I}^{T}
$$

Finally, in the case of a constant coefficient matrix $\mathcal{L}$, above system (52) can be re-written as a new symmetrised system of hyperbolic equations defined by

$$
\frac{\partial \mathcal{W}}{\partial t}+\hat{\mathcal{A}}_{I} \frac{\partial \mathcal{W}}{\partial X_{I}}=\hat{\mathcal{S}}
$$

where

$$
\mathcal{V}=\mathcal{L}^{-T} \mathcal{W} ; \quad \mathcal{S}=\mathcal{L} \hat{\mathcal{S}}
$$

As presented in [72], in order to develop a correct multi-dimensional generalisation of the Streamline Upwind Petrov-Galerkin (SUPG) for the system (48), it is useful to first derive the SUPG-type formulation for the system (53) and then map the answer back to (48) with the aid of the transformation matrix $\mathcal{L}$.

\subsection{Conservation of energy and generalised convex entropy}

In order to derive a suitable generalised entropy and entropy flux functions for the elastodynamic system at hand, consider the following convex entropy function defined by:

$$
S(\boldsymbol{p}, \boldsymbol{F}, \boldsymbol{H}, J)=\frac{1}{2 \rho_{0}} \boldsymbol{p} \cdot \boldsymbol{p}+W(\boldsymbol{F}, \boldsymbol{H}, J)
$$

which clearly represents the kinetic and elastic energy per unit undeformed volume. The corresponding flux vector can be easily shown to be given by

$$
\boldsymbol{\Lambda}=-\frac{1}{\rho_{0}} \boldsymbol{P}^{T} \boldsymbol{p} .
$$

In order to prove this, note first that the conjugate entropy variables are given by the derivatives of $S$ as,

$$
\mathcal{V}=\frac{\partial S}{\partial \mathcal{U}}=\left[\begin{array}{c}
\boldsymbol{v} \\
\boldsymbol{\Sigma}_{\boldsymbol{F}} \\
\boldsymbol{\Sigma}_{\boldsymbol{H}} \\
\Sigma_{J}
\end{array}\right]
$$


Multiplying each of the conservation laws $((10),(45))$ by the corresponding conjugate variables (57), and using the involution equations (6) for the deformation gradient and its co-factor, gives:

$$
\begin{aligned}
\frac{\partial S}{\partial t} & =\boldsymbol{v} \cdot \frac{\partial \boldsymbol{p}}{\partial t}+\boldsymbol{\Sigma}_{\boldsymbol{F}}: \frac{\partial \boldsymbol{F}}{\partial t}+\boldsymbol{\Sigma}_{\boldsymbol{H}}: \frac{\partial \boldsymbol{H}}{\partial t}+\Sigma_{J} \frac{\partial J}{\partial t} \\
& =\boldsymbol{v} \cdot \operatorname{DIVP}+\boldsymbol{\Sigma}_{\boldsymbol{F}}: \boldsymbol{\nabla}_{0} \boldsymbol{v}+\boldsymbol{\Sigma}_{\boldsymbol{H}}: \operatorname{CURL}(\boldsymbol{v} \times \boldsymbol{F})+\Sigma_{J} \operatorname{DIV}\left(\boldsymbol{H}^{T} \boldsymbol{v}\right) \\
& =\boldsymbol{v} \cdot \operatorname{DIVP}+\boldsymbol{\Sigma}_{\boldsymbol{F}}: \boldsymbol{\nabla}_{0} \boldsymbol{v}+\boldsymbol{\Sigma}_{\boldsymbol{H}}:\left(\boldsymbol{F} \times \boldsymbol{\nabla}_{0} \boldsymbol{v}\right)+\Sigma_{J} \boldsymbol{H}: \boldsymbol{\nabla}_{0} \boldsymbol{v} \\
& =\boldsymbol{v} \cdot \operatorname{DIVP}+\left(\boldsymbol{\Sigma}_{\boldsymbol{F}}+\boldsymbol{\Sigma}_{\boldsymbol{H}} \times \boldsymbol{F}+\Sigma_{J} \boldsymbol{H}\right): \boldsymbol{\nabla}_{0} \boldsymbol{v} \\
& =\boldsymbol{v} \cdot \operatorname{DIVP}+\boldsymbol{P}: \boldsymbol{\nabla}_{0} \boldsymbol{v} \\
& =\operatorname{DIV}\left(\boldsymbol{P}^{T} \boldsymbol{v}\right) .
\end{aligned}
$$

This statement is in fact a simplified version of the energy conservation law, or first law of thermodynamics, which, in the absence of the heat sources and heat flow, is globally stated as:

$$
\frac{d}{d t} \int_{V} E d V=\int_{\partial V} \boldsymbol{t}_{0} \cdot \boldsymbol{v} d A+\int_{V} \boldsymbol{f}_{0} \cdot \boldsymbol{v} d V
$$

where $E$ denotes the total energy per unit undeformed volume. The local version of this equation gives,

$$
\frac{\partial E}{\partial t}-\mathbf{D I V}\left(\boldsymbol{P}^{T} \boldsymbol{v}\right)=\boldsymbol{f}_{0} \cdot \boldsymbol{v}
$$

It is therefore clear that for the isothermal case under consideration, the generalised entropy is simply the total energy per unit undeformed volume minus the external energy component due to the body forces, that is, $S=$ $E-\boldsymbol{f}_{0} \cdot \boldsymbol{x}$, coinciding with the notion of Hamiltonian per unit of undeformed volume.

\subsection{Symmetric hyperbolic equations for elastodynamics}

With the definition of the conjugate entropy variables given above (57), it is now possible to derive a symmetric quasi-linear system for the velocity and conjugate stresses, as in (48). In order to do this, consider first the linear momentum equation. This equation can now be transformed taking

advantage of the fact that the deformation gradient is curl free and the area 
map divergence free as follows:

$$
\begin{aligned}
\boldsymbol{f}_{0} & =\rho_{0} \frac{\partial \boldsymbol{v}}{\partial t}-\operatorname{DIVP} \\
& =\rho_{0} \frac{\partial \boldsymbol{v}}{\partial t}-\operatorname{DIV}\left(\boldsymbol{\Sigma}_{\boldsymbol{F}}+\boldsymbol{\Sigma}_{\boldsymbol{H}} \times \boldsymbol{F}+\Sigma_{J} \boldsymbol{H}\right) \\
& =\rho_{0} \frac{\partial \boldsymbol{v}}{\partial t}-\operatorname{DIV} \boldsymbol{\Sigma}_{\boldsymbol{F}}+\boldsymbol{F}_{\boldsymbol{\Sigma}} \times \mathbf{C U R L} \boldsymbol{\Sigma}_{\boldsymbol{H}}-\boldsymbol{H}_{\boldsymbol{\Sigma}} \boldsymbol{\nabla}_{0} \Sigma_{J}
\end{aligned}
$$

where the notations $\boldsymbol{F}_{\boldsymbol{\Sigma}}$ and $\boldsymbol{H}_{\boldsymbol{\Sigma}}$ have been used here to explicitly indicate that these tensors are now being evaluated from the conjugate stresses using the reverse constitutive relationships, namely $\boldsymbol{F}_{\boldsymbol{\Sigma}}=\boldsymbol{F}\left(\boldsymbol{\Sigma}_{\boldsymbol{F}}, \boldsymbol{\Sigma}_{\boldsymbol{H}}, \Sigma_{J}\right)$ and $\boldsymbol{H}_{\boldsymbol{\Sigma}}=\boldsymbol{H}\left(\boldsymbol{\Sigma}_{\boldsymbol{F}}, \boldsymbol{\Sigma}_{\boldsymbol{H}}, \Sigma_{J}\right)$. For the Mooney-Rivlin model under consideration, the stress-strain relationships are decoupled, resulting in $\boldsymbol{F}_{\boldsymbol{\Sigma}}=\frac{1}{2 \alpha} \boldsymbol{\Sigma}_{\boldsymbol{F}}, \boldsymbol{H}_{\boldsymbol{\Sigma}}=$ $\frac{1}{2 \beta} \boldsymbol{\Sigma}_{\boldsymbol{H}}$.

The conservation laws for the strain measures can be easily transformed into equivalent equations expressed in terms of the conjugate stresses. For instance, the equation for the deformation gradient becomes,

$$
\frac{1}{2 \alpha} \frac{\partial \boldsymbol{\Sigma}_{\boldsymbol{F}}}{\partial t}-\boldsymbol{\nabla}_{0} \boldsymbol{v}=\mathbf{0}
$$

Similarly, the equation for the area map is transformed to give,

$$
\frac{1}{2 \beta} \frac{\partial \boldsymbol{\Sigma}_{\boldsymbol{H}}}{\partial t}-\boldsymbol{F}_{\boldsymbol{\Sigma}} \times \boldsymbol{\nabla}_{0} \boldsymbol{v}=\mathbf{0}
$$

Note that for the Neo-Hookean case, where $\beta=0$, the corresponding conjugate stress $\boldsymbol{\Sigma}_{\boldsymbol{H}}=\mathbf{0}$ and the above equation is not part of the system as the generalised entropy $S(\boldsymbol{p}, \boldsymbol{F}, J)$ is no longer a function of $\boldsymbol{H}$. Finally the volume rate equation becomes,

$$
\frac{1}{\gamma} \frac{\partial \Sigma_{J}}{\partial t}-\boldsymbol{H}_{\Sigma}: \nabla_{0} \boldsymbol{v}=0 ; \quad \frac{1}{\gamma}=\frac{d J}{d \Sigma_{J}}=\frac{\partial^{2} \Upsilon}{\partial \Sigma_{J}^{2}}
$$

For completeness, the matrix $\mathcal{A}_{0}$ (49) is presented below

$$
\mathcal{A}_{0}=\left[\begin{array}{cccc}
\rho_{0} \boldsymbol{I} & \mathbf{0} & \mathbf{0} & \mathbf{0} \\
\mathbf{0} & \frac{1}{2 \alpha} \mathcal{I} & \mathbf{0} & \mathbf{0} \\
\mathbf{0} & \mathbf{0} & \frac{1}{2 \beta} \mathcal{I} & \mathbf{0} \\
\mathbf{0} & \mathbf{0} & \mathbf{0} & \frac{1}{\gamma}
\end{array}\right]
$$


with $\mathcal{I}$ the fourth order identity tensor. The combined set of symmetric hyperbolic equations for the conjugate entropy variables, as in (48), can now be written as:

$$
\begin{array}{r}
\rho_{0} \frac{\partial \boldsymbol{v}}{\partial t}-\operatorname{DIV}_{\boldsymbol{F}}+\boldsymbol{F}_{\boldsymbol{\Sigma}} \times \mathbf{C U R L} \boldsymbol{\Sigma}_{\boldsymbol{H}}-\boldsymbol{H}_{\boldsymbol{\Sigma}} \boldsymbol{\nabla}_{0} \Sigma_{J}=\boldsymbol{f}_{0} \\
\frac{1}{2 \alpha} \frac{\partial \boldsymbol{\Sigma}_{\boldsymbol{F}}}{\partial t}-\boldsymbol{\nabla}_{0} \boldsymbol{v}=\mathbf{0} \\
\frac{1}{2 \beta} \frac{\partial \boldsymbol{\Sigma}_{\boldsymbol{H}}}{\partial t}-\boldsymbol{F}_{\boldsymbol{\Sigma}} \times \nabla_{0} \boldsymbol{v}=\mathbf{0} \\
\frac{1}{\gamma} \frac{\partial \Sigma_{J}}{\partial t}-\boldsymbol{H}_{\boldsymbol{\Sigma}}: \boldsymbol{\nabla}_{0} \boldsymbol{v}=0
\end{array}
$$

The symmetric nature of this system is more easily appreciated using indicial notation and rewriting the above set of equations as:

$$
\frac{\partial}{\partial t}\left[\begin{array}{c}
\rho_{0} v_{i} \\
\frac{1}{2 \alpha}\left[\boldsymbol{\Sigma}_{\boldsymbol{F}}\right]_{i J} \\
\frac{1}{2 \beta}\left[\boldsymbol{\Sigma}_{\boldsymbol{H}}\right]_{i J} \\
\frac{1}{\gamma} \Sigma_{J}
\end{array}\right]-\left[\begin{array}{cccc}
\mathbf{0}_{3 \times 3} & \delta_{i k} \delta_{K I} & \mathcal{E}_{i j k} \mathcal{E}_{I J K} F_{j J} & H_{i I} \\
\delta_{i k} \delta_{J I} & \mathbf{0}_{3 \times 3 \times 3 \times 3} & \mathbf{0}_{3 \times 3 \times 3 \times 3} & \mathbf{0}_{3 \times 3} \\
\mathcal{E}_{i j k} \mathcal{E}_{J K I} F_{j K} & \mathbf{0}_{3 \times 3 \times 3 \times 3} & \mathbf{0}_{3 \times 3 \times 3 \times 3} & \mathbf{0}_{3 \times 3} \\
H_{k I} & \mathbf{0}_{3 \times 3} & \mathbf{0}_{3 \times 3} & 0
\end{array}\right] \frac{\partial}{\partial X_{I}}\left[\begin{array}{c}
v_{k} \\
{\left[\boldsymbol{\Sigma}_{\boldsymbol{F}}\right]_{k K}} \\
{\left[\boldsymbol{\Sigma}_{\boldsymbol{H}}\right]_{k K}} \\
\Sigma_{J}
\end{array}\right]=\left[\begin{array}{c}
\boldsymbol{f}_{0} \\
\mathbf{0} \\
\mathbf{0} \\
0
\end{array}\right]
$$

The above set of symmetric equations (66) for the entropy conjugate variables can also be written in the transformed form (51) as:

$$
\begin{aligned}
\frac{\partial \boldsymbol{v}}{\partial t}-\frac{1}{\rho_{0}}\left(\mathbf{D I V} \boldsymbol{\Sigma}_{\boldsymbol{F}}-\boldsymbol{F}_{\boldsymbol{\Sigma}} \times \mathbf{C U R L} \boldsymbol{\Sigma}_{\boldsymbol{H}}+\boldsymbol{H}_{\boldsymbol{\Sigma}} \boldsymbol{\nabla}_{0} \Sigma_{J}\right) & =\frac{1}{\rho_{0}} \boldsymbol{f}_{0} \\
\frac{\partial \boldsymbol{\Sigma}_{\boldsymbol{F}}}{\partial t}-2 \alpha \boldsymbol{\nabla}_{0} \boldsymbol{v} & =\mathbf{0} \\
\frac{\partial \boldsymbol{\Sigma}_{\boldsymbol{H}}}{\partial t}-2 \beta \boldsymbol{F}_{\boldsymbol{\Sigma}} \times \boldsymbol{\nabla}_{0} \boldsymbol{v} & =\mathbf{0} \\
\frac{\partial \Sigma_{J}}{\partial t}-\gamma \boldsymbol{H}_{\boldsymbol{\Sigma}}: \boldsymbol{\nabla}_{0} \boldsymbol{v} & =0 .
\end{aligned}
$$

\subsection{Eigenvalue structure}

The eigenvalues or wave speeds and corresponding eigenvectors of the above system can be determined by identifying possible plane wave solutions 
(in the absence of source terms) of the type:

$$
\mathcal{V}=\overline{\mathcal{V}}_{\alpha} \phi\left(\boldsymbol{X} \cdot \boldsymbol{N}-c_{\alpha} t\right)=\phi\left(\boldsymbol{X} \cdot \boldsymbol{N}-c_{\alpha} t\right)\left[\begin{array}{c}
\overline{\boldsymbol{v}}_{\alpha} \\
\overline{\boldsymbol{\Sigma}}_{\boldsymbol{F}}^{\alpha} \\
\overline{\boldsymbol{\Sigma}}_{\boldsymbol{H}}^{\alpha} \\
\bar{\Sigma}_{J}^{\alpha}
\end{array}\right]
$$

where $c_{\alpha}$ are the wave speeds corresponding to the eigenmode $\overline{\mathcal{V}}_{\alpha}$ and $\boldsymbol{N}$ the direction of propagation. It is easy to show that the above expression for the conjugate variables leads to an eigenvalue problem (refer to equation (48)) given by,

$$
\tilde{\mathcal{A}}_{N} \overline{\mathcal{V}}_{\alpha}=c_{\alpha} \mathcal{A}_{0} \overline{\mathcal{V}}_{\alpha} ; \quad \quad \tilde{\mathcal{A}}_{N}=\tilde{\mathcal{A}}_{I} N_{I}
$$

Notice that the use of equations $(12)_{1}$ or (53) can lead to alternative representations of the eigenvalue problem as follows

$$
\begin{aligned}
\mathcal{A}_{N} \overline{\mathcal{U}}_{\alpha} & =c_{\alpha} \overline{\mathcal{U}}_{\alpha} ; & \mathcal{A}_{\boldsymbol{N}} & =\mathcal{A}_{I} N_{I} \\
\hat{\mathcal{A}}_{N} & \overline{\mathcal{W}}_{\alpha}=c_{\alpha} \overline{\mathcal{W}}_{\alpha} ; & \hat{\mathcal{A}}_{\boldsymbol{N}} & =\hat{\mathcal{A}}_{I} N_{I}
\end{aligned}
$$

with identical eigenvalues $c_{\alpha}$ and eigenvectors related as follows

$$
\overline{\mathcal{U}}_{\alpha}=\mathcal{A}_{0} \overline{\mathcal{V}}_{\alpha}=\mathcal{L} \overline{\mathcal{W}}_{\alpha}
$$

It is far simpler to deal with the resulting eigen-problem by considering each individual component of this system. For this purpose, note first that the time derivative, gradient, divergence and curl operators for the proposed plane wave solution become,

$$
\begin{aligned}
\frac{\partial \mathcal{V}}{\partial t} & =-c_{\alpha} \overline{\mathcal{V}}_{\alpha} \phi^{\prime} \\
\boldsymbol{\nabla}_{0} \boldsymbol{v} & =\left(\overline{\boldsymbol{v}}_{\alpha} \otimes \boldsymbol{N}\right) \phi^{\prime} \\
\operatorname{DIV} \boldsymbol{\Sigma}_{\boldsymbol{F}} & =\left(\overline{\boldsymbol{\Sigma}}_{\boldsymbol{F}}^{\alpha} \boldsymbol{N}\right) \phi^{\prime} \\
\mathbf{C U R L} \boldsymbol{\Sigma}_{\boldsymbol{H}} & =-\left(\overline{\boldsymbol{\Sigma}}_{\boldsymbol{H}}^{\alpha} \times \boldsymbol{N}\right) \phi^{\prime} \\
\boldsymbol{\nabla}_{0} \Sigma_{J} & =\left(\bar{\Sigma}_{J}^{\alpha} \boldsymbol{N}\right) \phi^{\prime} .
\end{aligned}
$$

Using these expressions together with equations in (66) and the properties of the vector and tensor cross products given in Appendix A, gives after some 
simple algebra:

$$
\begin{aligned}
-\left(\overline{\boldsymbol{\Sigma}}_{\boldsymbol{F}}^{\alpha}+\boldsymbol{F} \times \overline{\boldsymbol{\Sigma}}_{\boldsymbol{H}}^{\alpha}+\bar{\Sigma}_{J}^{\alpha} \boldsymbol{H}\right) \boldsymbol{N} & =\rho_{0} c_{\alpha} \overline{\boldsymbol{v}}_{\alpha} \\
-2 \alpha\left(\overline{\boldsymbol{v}}_{\alpha} \otimes \boldsymbol{N}\right) & =c_{\alpha} \overline{\boldsymbol{\Sigma}}_{\boldsymbol{F}}^{\alpha} \\
-2 \beta \boldsymbol{F} \times\left(\overline{\boldsymbol{v}}_{\alpha} \otimes \boldsymbol{N}\right) & =c_{\alpha} \overline{\boldsymbol{\Sigma}}_{\boldsymbol{H}}^{\alpha} \\
-\gamma\left(\overline{\boldsymbol{v}}_{\alpha} \cdot \boldsymbol{H} \boldsymbol{N}\right) & =c_{\alpha} \bar{\Sigma}_{J}^{\alpha} .
\end{aligned}
$$

As a consequence of the high level of redundancy in the system of equations being considered, only 6 wave speeds are different from zero. These can be readily identified by substituting the last three equations for the conjugate stresses into the first one to give an eigenvalue problem for the velocity component alone as:

$$
2 \alpha \overline{\boldsymbol{v}}_{\alpha}+\gamma \boldsymbol{H} \boldsymbol{N}\left(\overline{\boldsymbol{v}}_{\alpha} \cdot \boldsymbol{H} \boldsymbol{N}\right)+2 \beta\left[\boldsymbol{F} \times\left(\boldsymbol{F} \times\left(\overline{\boldsymbol{v}}_{\alpha} \otimes \boldsymbol{N}\right)\right)\right] \boldsymbol{N}=\rho_{0} c_{\alpha}^{2} \overline{\boldsymbol{v}}_{\alpha} .
$$

The double tensor cross product term can be simplified by repeated use of the third order alternating tensor product expression $\mathcal{E}_{i j k} \mathcal{E}_{l m k}=\delta_{i l} \delta_{j m}-\delta_{i m} \delta_{j l}$ and noting that:

$$
\boldsymbol{I}-\boldsymbol{N} \otimes \boldsymbol{N}=\boldsymbol{T}_{1} \otimes \boldsymbol{T}_{1}+\boldsymbol{T}_{2} \otimes \boldsymbol{T}_{2}
$$

where $\boldsymbol{I}$ is the identity matrix and $\boldsymbol{T}_{1,2}$ denote an arbitrary pair of orthogonal unit vectors on the reference propagation plane with normal $\boldsymbol{N}$. With the help of these expressions the eigenvalue problem becomes:

$$
2 \alpha \overline{\boldsymbol{v}}_{\alpha}+\gamma \Lambda_{A}^{2} \boldsymbol{n}\left(\overline{\boldsymbol{v}}_{\alpha} \cdot \boldsymbol{n}\right)+2 \beta\left(\Lambda_{T}^{2} \overline{\boldsymbol{v}}_{\alpha}-\boldsymbol{\Lambda}_{T} \overline{\boldsymbol{v}}_{\alpha}\right)=\rho_{0} c_{\alpha} \overline{\boldsymbol{v}}_{\alpha}
$$

where the following notation has been used:

$$
\begin{aligned}
\Lambda_{A} \boldsymbol{n} & =\boldsymbol{H} \boldsymbol{N} \\
\Lambda_{A}^{2} & =\boldsymbol{H} \boldsymbol{N} \cdot \boldsymbol{H} \boldsymbol{N} \\
\Lambda_{T} & =\boldsymbol{F} \boldsymbol{T}_{1} \otimes \boldsymbol{F} \boldsymbol{T}_{1}+\boldsymbol{F} \boldsymbol{T}_{2} \otimes \boldsymbol{F} \boldsymbol{T}_{2} \\
\Lambda_{T}^{2} & =\boldsymbol{F} \boldsymbol{T}_{1} \cdot \boldsymbol{F} \boldsymbol{T}_{1}+\boldsymbol{F} \boldsymbol{T}_{2} \cdot \boldsymbol{F} \boldsymbol{T}_{2}=\operatorname{tr} \Lambda_{T} .
\end{aligned}
$$

Note that by construction $\boldsymbol{n}$ is a unit vector orthogonal to the vectors $\boldsymbol{F} \boldsymbol{T}_{1,2}$ which lie on the propagation surface. The first set of eigenvectors/eigenvalues corresponding to $p$-waves is obtained by taking $\overline{\boldsymbol{v}}=\boldsymbol{n}$ to give,

$$
c_{1,2}= \pm \sqrt{\frac{2 \alpha+2 \beta \Lambda_{T}^{2}+\gamma \Lambda_{A}^{2}}{\rho_{0}}} .
$$


The associated eigenvectors are obtained from equation (74) to give:

$$
\overline{\mathcal{V}}_{1,2}=\left[\begin{array}{c}
\boldsymbol{n} \\
-\frac{2 \alpha}{c_{1,2}} \boldsymbol{n} \otimes \boldsymbol{N} \\
-\frac{2 \beta}{c_{1,2}} \boldsymbol{F} \times(\boldsymbol{n} \otimes \boldsymbol{N}) \\
-\frac{\gamma}{c_{1,2}} \boldsymbol{n} \cdot \boldsymbol{H} \boldsymbol{N}
\end{array}\right]
$$

The next four eigenvalues correspond to shear waves where the vibration takes place on the propagation plane. The corresponding velocity vectors are orthogonal to $\boldsymbol{n}$ and in the directions of the unit eigenvectors $\left\{\boldsymbol{t}_{1}, \boldsymbol{t}_{2}\right\}$ of the rank two tensor $\boldsymbol{\Lambda}_{T}$. The wave speeds are given by:

$$
c_{3,4}= \pm \sqrt{\frac{2 \alpha+2 \beta\left(\Lambda_{T}^{2}-\lambda_{1}^{2}\right)}{\rho_{0}}} ; \quad c_{5,6}= \pm \sqrt{\frac{2 \alpha+2 \beta\left(\Lambda_{T}^{2}-\lambda_{2}^{2}\right)}{\rho_{0}}}
$$

where $\lambda_{1,2}^{2}$ are the eigenvalues of $\boldsymbol{\Lambda}_{T}$, that is:

$$
\boldsymbol{\Lambda}_{T}=\lambda_{1}^{2} \boldsymbol{t}_{1} \otimes \boldsymbol{t}_{1}+\lambda_{2}^{2} \boldsymbol{t}_{2} \otimes \boldsymbol{t}_{2} .
$$

The corresponding eigenvectors are given by:

$$
\overline{\mathcal{V}}_{3,4}=\left[\begin{array}{c}
\boldsymbol{t}_{1} \\
-\frac{2 \alpha}{c_{3,4}} \boldsymbol{t}_{1} \otimes \boldsymbol{N} \\
-\frac{2 \beta}{c_{3,4}} \boldsymbol{F} \times\left(\boldsymbol{t}_{1} \otimes \boldsymbol{N}\right) \\
0
\end{array}\right] ; \quad \overline{\mathcal{V}}_{5,6}=\left[\begin{array}{c}
\boldsymbol{t}_{2} \\
-\frac{2 \alpha}{c_{5,6}} \boldsymbol{t}_{2} \otimes \boldsymbol{N} \\
-\frac{2 \beta}{c_{5,6}} \boldsymbol{F} \times\left(\boldsymbol{t}_{2} \otimes \boldsymbol{N}\right) \\
0
\end{array}\right]
$$

The remainder of the eigenvalues of the system (74) are zero and have null associated velocity component. The stress components $\left\{\overline{\boldsymbol{\Sigma}}_{\boldsymbol{F}}^{\alpha}, \overline{\boldsymbol{\Sigma}}_{\boldsymbol{H}}^{\alpha}, \bar{\Sigma}_{J}^{\alpha}\right\}$ of their associated eigenvectors satisfy the vector condition:

$$
\left(\overline{\boldsymbol{\Sigma}}_{\boldsymbol{F}}^{\alpha}+\boldsymbol{F} \times \overline{\boldsymbol{\Sigma}}_{\boldsymbol{H}}^{\alpha}+\bar{\Sigma}_{J}^{\alpha} \boldsymbol{H}\right) \boldsymbol{N}=\mathbf{0} .
$$

This represents three linear conditions for 19 stress variables, thereby giving a null subspace of dimension 16 to complete the 6 real wave speeds derived above. 


\section{Finite element implementation}

The set of equations described above can be discretised in a variety of different ways. Integral conservation laws will be useful for cell based finite volume type of approximation [50-52, 84] whereas the local equations for the conservation variables or the entropy variables are typically discretised using stabilised Petrov-Galerkin finite element techniques $[1,8]$. Some possible implementations of these techniques are described in the section below but reader should also refer to the numerous works by other authors in this field $[8,72,83,85-95]$.

\subsection{General remarks}

Consider a standard finite element partition of the domain into a set of elements. Inside each element the problem variables (i.e. $\mathcal{U}$ or $\mathcal{V}$ ) are interpolated in terms of a set of shape functions $N_{a}^{\mathcal{U}}$ or $N_{a}^{\mathcal{V}}$ as:

$$
\boldsymbol{p}=\sum_{a=1}^{n_{\boldsymbol{p}}} \boldsymbol{p}_{a} N_{a}^{\boldsymbol{p}} ; \quad \boldsymbol{F}=\sum_{a=1}^{n_{\boldsymbol{F}}} \boldsymbol{F}_{a} N_{a}^{\boldsymbol{F}} ; \quad \ldots ; \quad \boldsymbol{\Sigma}_{\boldsymbol{F}}=\sum_{a=1}^{n_{\boldsymbol{\Sigma}_{\boldsymbol{F}}}} \boldsymbol{\Sigma}_{\boldsymbol{F}}^{a} N_{a}^{\boldsymbol{\Sigma}_{\boldsymbol{F}}}
$$

where $a$ denotes the nodes or other degrees of freedom used in the interpolation of the above variables. In general, different interpolations can be (and are often) used to describe different variables. However, the same interpolation space will be used for conjugate pairs.

The residual $\mathcal{R}$ of each conservation law can either be expressed in terms of conservation variables, namely $\mathcal{R}=\mathcal{R}(\mathcal{U})$

$$
\mathcal{R}=\left[\begin{array}{c}
\mathcal{R}_{p} \\
\mathcal{R}_{\boldsymbol{F}} \\
\mathcal{R}_{\boldsymbol{H}} \\
\mathcal{R}_{J}
\end{array}\right]=\left[\begin{array}{c}
\frac{\partial \boldsymbol{p}}{\partial t}-\operatorname{DIV} \boldsymbol{P}-\boldsymbol{f}_{0} \\
\frac{\partial \boldsymbol{F}}{\partial t}-\operatorname{DIV}\left(\frac{1}{\rho_{0}} \boldsymbol{p} \otimes \boldsymbol{I}\right) \\
\frac{\partial \boldsymbol{H}}{\partial t}-\operatorname{CURL}\left(\frac{1}{\rho_{0}} \boldsymbol{p} \times \boldsymbol{F}\right) \\
\frac{\partial J}{\partial t}-\operatorname{DIV}\left(\frac{1}{\rho_{0}} \boldsymbol{H}^{T} \boldsymbol{p}\right)
\end{array}\right]
$$


or their entropy conjugates, namely $\mathcal{R}=\mathcal{R}(\mathcal{V})$, as:

$$
\boldsymbol{R}=\left[\begin{array}{c}
\mathcal{R}_{p} \\
\mathcal{R}_{\boldsymbol{F}} \\
\boldsymbol{\mathcal { R }}_{\boldsymbol{H}} \\
\mathcal{R}_{J}
\end{array}\right]=\left[\begin{array}{c}
\rho_{0} \frac{\partial \boldsymbol{v}}{\partial t}-\mathrm{DIV}_{\boldsymbol{F}}+\boldsymbol{F}_{\boldsymbol{\Sigma}} \times \operatorname{CURL} \boldsymbol{\Sigma}_{\boldsymbol{H}}-\boldsymbol{H}_{\boldsymbol{\Sigma}} \boldsymbol{\nabla}_{0} \Sigma_{J}-\boldsymbol{f}_{0} \\
\frac{1}{2 \alpha} \frac{\partial \boldsymbol{\Sigma}_{\boldsymbol{F}}}{\partial t}-\boldsymbol{\nabla}_{0} \boldsymbol{v} \\
\frac{1}{2 \beta} \frac{\partial \boldsymbol{\Sigma}_{\boldsymbol{H}}}{\partial t}-\boldsymbol{F}_{\boldsymbol{\Sigma}} \times \boldsymbol{\nabla}_{0} \boldsymbol{v} \\
\frac{1}{\gamma} \frac{\partial \Sigma_{J}}{\partial t}-\boldsymbol{H}_{\boldsymbol{\Sigma}}: \boldsymbol{\nabla}_{0} \boldsymbol{v}
\end{array}\right]
$$

A standard Galerkin approximation of the elasto-dynamics equations is established by the integral condition:

$$
\int_{V} \delta \mathcal{V}^{T} \boldsymbol{\mathcal { R }} d V=\int_{V}\left(\delta \boldsymbol{v} \cdot \boldsymbol{\mathcal { R }}_{\boldsymbol{p}}+\delta \boldsymbol{\Sigma}_{\boldsymbol{F}}: \boldsymbol{\mathcal { R }}_{\boldsymbol{F}}+\delta \boldsymbol{\Sigma}_{\boldsymbol{H}}: \boldsymbol{\mathcal { R }}_{\boldsymbol{H}}+\delta \Sigma_{J} \mathcal{R}_{J}\right) d V=0
$$

for all $\delta \mathcal{V}=\left[\delta \boldsymbol{v}, \delta \boldsymbol{\Sigma}_{\boldsymbol{F}}, \delta \boldsymbol{\Sigma}_{\boldsymbol{H}}, \delta \Sigma_{J}\right]^{T}$ compatible with the boundary conditions.

Remark 1: Note that, whether conservation $\mathcal{U}$ or entropy variables $\mathcal{V}$ are used in order to represent the residual equations (i.e. $\mathcal{R}(\mathcal{U})$ or $\mathcal{R}(\mathcal{V})$ ), it is critical that each residual component is weighted by the appropriate virtual conjugate variable $\delta \mathcal{V}$ in order to ensure that the physical units of the products are compatible and can be added together. Crucially [64], this also ensures that the numerical procedure inherits the entropy production inequality of the continuum system for both conservation and entropy variables, as can be easily observed by choosing the actual values of the conjugate variables as virtual weights in the above expression.

In the Computational Fluid Dynamics (CFD) community, a transformation to non-dimensional variables, instead of entropy variables, is often used. In particular, a set of dimensionless conservation variables $\overline{\mathcal{U}}$ is defined via a linear transformation $\overline{\mathcal{U}}=\overline{\mathcal{A}}_{0} \mathcal{U}$ where $\overline{\mathcal{A}}_{0}$ is a diagonal matrix of appropriate physical constants that render the system dimensionless. The term $\int_{V} \delta \overline{\mathcal{U}}^{T} \overline{\mathcal{R}} d V$ is well defined (i.e. all its contributions are dimensionless) but will not preserve the entropy production properties of the continuum.

The use of conservation variables in equation (88) is typically accompanied by an integration by parts of the divergence of the flux operator. This 
gives:

$$
\begin{aligned}
0 & =\int_{V} \delta \mathcal{V}^{T} \boldsymbol{R} d V \\
& =\int_{V} \delta \mathcal{V}^{T}\left(\frac{\partial \mathcal{U}}{\partial t}+\operatorname{DIV} \mathcal{F}-\mathcal{S}\right) d V \\
& =\int_{V} \delta \mathcal{V}^{T} \frac{\partial \mathcal{U}}{\partial t} d V+\int_{\partial V} \delta \mathcal{V}^{T} \mathcal{F}_{I} N_{I} d A-\int_{V} \mathcal{F}_{I} \frac{\partial \delta \mathcal{V}}{\partial X_{I}} d V-\int_{V} \delta \mathcal{V}^{T} \mathcal{S} d V
\end{aligned}
$$

The individual components of this equation (89) correspond to the principle of virtual work for the case of the linear momentum equation:

$$
\int_{V} \delta \boldsymbol{v} \cdot \frac{\partial \boldsymbol{p}}{\partial t} d V=\int_{\partial_{t} V} \delta \boldsymbol{v} \cdot \boldsymbol{t}_{B} d A+\int_{V} \delta \boldsymbol{v} \cdot \boldsymbol{f}_{0} d V-\int_{V} \boldsymbol{P}: \boldsymbol{\nabla}_{0} \delta \boldsymbol{v} d V
$$

whereas the three geometric conservation laws are now re-expressed as:

$$
\begin{aligned}
\int_{V} \delta \boldsymbol{\Sigma}_{\boldsymbol{F}}: \frac{\partial \boldsymbol{F}}{\partial t} d V & =\int_{\partial_{u} V} \boldsymbol{v}_{B} \cdot \delta \boldsymbol{\Sigma}_{\boldsymbol{F}} \boldsymbol{N} d A-\int_{V} \frac{1}{\rho_{0}} \boldsymbol{p} \cdot \operatorname{DIV}\left(\delta \boldsymbol{\Sigma}_{\boldsymbol{F}}\right) d V \\
\int_{V} \delta \boldsymbol{\Sigma}_{\boldsymbol{H}}: \frac{\partial \boldsymbol{H}}{\partial t} d V & =-\int_{\partial_{u} V}\left(\boldsymbol{v}_{B} \times \boldsymbol{F} \times \boldsymbol{N}\right): \delta \boldsymbol{\Sigma}_{\boldsymbol{H}} d A+\int_{V}\left(\frac{1}{\rho_{0}} \boldsymbol{p} \times \boldsymbol{F}\right): \mathbf{C U R L}\left(\delta \boldsymbol{\Sigma}_{\boldsymbol{H}}\right) d V \\
\int_{V} \delta \Sigma_{J} \frac{\partial J}{\partial t} d V & =\int_{\partial_{u} V}\left(\boldsymbol{v}_{B} \cdot \boldsymbol{H} \boldsymbol{N}\right) \delta \Sigma_{J} d A-\int_{V} \frac{1}{\rho_{0}} \boldsymbol{p} \cdot \boldsymbol{H} \boldsymbol{\nabla}_{0}\left(\delta \Sigma_{J}\right) d V .
\end{aligned}
$$

In these expressions, the velocity and traction at the boundary, namely $\boldsymbol{v}_{B}$ and $\boldsymbol{t}_{B}$, respectively, can either be directly obtained from boundary conditions or through an appropriate Riemann solver [77, 96]. The main advantage of integrating by parts as shown above is to enable the imposition of the boundary conditions via boundary fluxes. This is indeed useful for the momentum equation (90) as it introduces naturally the boundary tractions, but less so in the case of geometric conservation laws (91). In addition, unless inter-element jumps are correctly taken into account, this integration by parts implies the use of $C_{0}$ continuous shape functions for the strains and stresses.

Alternatively, if only the linear momentum law is integrated by parts, it is possible to choose interpolation spaces for strains and stresses that are discontinuous across element boundaries, thus allowing these variables to be resolved element by element. In this way, and with a careful choice of interpolation spaces, it is possible to reproduce displacement-based formulations or the type of mixed formulations proposed in $[45,46]$. 
Furthermore, the fact that the momentum/velocity and strain/stress variables can be solved in a sequential manner, makes it possible to consider the momentum equation in conservation form integrated by parts and the other equations in the entropy form. This approach would not be dissimilar to the common methodology used by most hydrocodes [2] in which stress rates are obtained from velocity gradients. The proposed framework, however, has a firmer theoretical foundation and avoids issues such as frame invariance of stress rates.

\subsection{Time integration}

It is clear that a wide choice of finite element interpolation and time integration strategies is available for the equations described above. In the sections that follow two simple $C_{0}$ continuous linear tetrahedral interpolations for all the variables will be described. The resulting set of equations is rather large, so it will only be suitable for explicit type of time integrations, although semi-implicit staggered approaches in which only the velocities are resolved for implicitly will be explored in the future. For simplicity, an explicit Total Variation Diminishing (TVD) Runge-Kutta scheme, which has been widely used in the CFD context, is proposed at present [97-99]. This is described by the following time update equations from time step $t_{n}$ to time step $t_{n+1}$ with $\Delta t=t_{n+1}-t_{n}{ }^{4}$ :

$$
\begin{aligned}
& \mathcal{U}_{n+1}^{*}=\mathcal{U}_{n}+\Delta t \dot{\mathcal{U}}_{n} \\
& \mathcal{U}_{n+2}^{*}=\mathcal{U}_{n+1}^{*}+\Delta t \dot{\mathcal{U}}_{n+1}^{*} \\
& \mathcal{U}_{n+1}=\frac{1}{2}\left(\mathcal{U}_{n}+\mathcal{U}_{n+2}^{*}\right)
\end{aligned}
$$

which can be equally applied to conservation or entropy variables. Given the explicit nature of the scheme the time step is constrained by a CourantFriedrichs-Lewy (CFL) condition [100] given by:

$$
\Delta t \leq C F L \frac{h}{c_{\max }}
$$

where $c_{\max }$ corresponds to the speed of the pressure wave given in equation (79) and $h$ an elemental mesh size related parameter.

\footnotetext{
${ }^{4}$ As it is standard, the dot over a variable indicates differentiation with respect to time.
} 


\subsection{A stabilised Petrov-Galerkin discretisation of conservation variables us-} ing linear tetrahedra

As a simple example of the procedures described above, consider a linear tetrahedral discretisation of all conservation variables and their virtual conjugates in terms of linear $C_{0}$ shape functions $N_{a}$ as:

$\boldsymbol{p}=\sum_{a=1}^{4} \boldsymbol{p}_{a} N_{a} ; \quad \delta \boldsymbol{v}=\sum_{a=1}^{4} \delta \boldsymbol{v}_{a} N_{a} ; \quad \boldsymbol{F}=\sum_{a=1}^{4} \boldsymbol{F}_{a} N_{a} ; \quad \delta \boldsymbol{\Sigma}_{\boldsymbol{F}}=\sum_{a=1}^{4} \delta \boldsymbol{\Sigma}_{\boldsymbol{F}_{a}} N_{a} ; \quad \ldots$

In this case, the residual of the conservation laws is formulated with respect to the conservation variables $\mathcal{R}(\mathcal{U})(86)$. The Galerkin weighted residual equations described in (88) require stabilisation, which is usually introduced by means of a Petrov-Galerkin approach whereby the conjugate weighting functions $\delta \mathcal{V}$ are augmented as:

$$
\delta \mathcal{V}^{s t}=\delta \mathcal{V}+\boldsymbol{\tau}^{T} \mathcal{A}_{I}^{T} \frac{\partial \delta \mathcal{V}}{\partial X_{I}}
$$

where $\boldsymbol{\tau}$ denotes a matrix of stabilisation parameters to be described below. In the case of a diagonal stabilisation matrix $\boldsymbol{\tau}$, the individual components of this expression (95) are easily obtained with the help of equations (68) to give:

$$
\begin{aligned}
\delta \boldsymbol{v}^{s t} & =\delta \boldsymbol{v}-\frac{\tau_{\boldsymbol{v}}}{\rho_{0}}\left(\mathbf{D I V} \delta \boldsymbol{\Sigma}_{\boldsymbol{F}}-\boldsymbol{F} \times \mathbf{C U R L} \delta \boldsymbol{\Sigma}_{\boldsymbol{H}}+\boldsymbol{H} \boldsymbol{\nabla}_{0} \delta \Sigma_{J}\right) \\
\delta \boldsymbol{\Sigma}_{\boldsymbol{F}}^{s t} & =\delta \boldsymbol{\Sigma}_{\boldsymbol{F}}-2 \tau_{\boldsymbol{F}} \alpha\left(\boldsymbol{\nabla}_{0} \delta \boldsymbol{v}\right) \\
\delta \boldsymbol{\Sigma}_{\boldsymbol{H}}^{s t} & =\delta \boldsymbol{\Sigma}_{\boldsymbol{H}}-2 \tau_{\boldsymbol{H}} \beta\left(\boldsymbol{F} \times \boldsymbol{\nabla}_{0} \delta \boldsymbol{v}\right) \\
\delta \Sigma_{J}^{s t} & =\delta \Sigma_{J}-\tau_{J} \gamma\left(\boldsymbol{H}: \boldsymbol{\nabla}_{0} \delta \boldsymbol{v}\right) .
\end{aligned}
$$

where $\tau_{\boldsymbol{v}}, \tau_{\boldsymbol{F}}, \tau_{\boldsymbol{H}}$ and $\tau_{J}$ are appropriate stabilisation parameters. These stabilised weighting functions (96) can now be substituted into the residual equation (88) (i.e. replace $\delta \mathcal{V}$ with $\delta \mathcal{V}^{s t}$ ) and grouped according to each virtual conjugate variable. First, the terms containing the virtual velocity $\delta \boldsymbol{v}$ are given by:

$$
\int_{V}\left(\delta \boldsymbol{v} \cdot \mathcal{R}_{\boldsymbol{p}}-2 \tau_{\boldsymbol{F}} \alpha \boldsymbol{\mathcal { R }}_{\boldsymbol{F}}: \boldsymbol{\nabla}_{0} \delta \boldsymbol{v}-2 \tau_{\boldsymbol{H}} \beta\left(\boldsymbol{F} \times \nabla_{0} \delta \boldsymbol{v}\right): \boldsymbol{\mathcal { R }}_{\boldsymbol{H}}-\tau_{J} \gamma\left(\boldsymbol{H}: \boldsymbol{\nabla}_{0} \delta \boldsymbol{v}\right) \mathcal{R}_{J}\right) d V=0
$$


Integrating by parts the first term in (97), as described in equation (90), and recalling the constitutive equation for a compressible Mooney-Rivlin material gives:

$$
\int_{V} \delta \boldsymbol{v} \cdot \frac{\partial \boldsymbol{p}}{\partial t} d V=\int_{V} \delta \boldsymbol{v} \cdot \boldsymbol{f}_{0} d V+\int_{\partial_{t} V} \delta \boldsymbol{v} \cdot \boldsymbol{t}_{B} d A-\int_{V} \boldsymbol{P}^{s t}: \nabla_{0} \delta \boldsymbol{v} d V
$$

where the stabilised Piola-Kirchhoff stresses $\boldsymbol{P}^{s t}$ are:

$$
\begin{aligned}
\boldsymbol{P}^{s t} & =\boldsymbol{\Sigma}_{\boldsymbol{F}}^{s t}+\boldsymbol{\Sigma}_{\boldsymbol{H}}^{s t} \times \boldsymbol{F}+\Sigma_{J}^{s t} \boldsymbol{H} \\
\boldsymbol{\Sigma}_{\boldsymbol{F}}^{s t} & =2 \alpha \boldsymbol{F}^{s t} \\
\boldsymbol{\Sigma}_{\boldsymbol{H}}^{s t} & =2 \beta \boldsymbol{H}^{s t} \\
\Sigma_{J}^{s t} & =\Sigma_{J}(J)-\tau_{J} \gamma \mathcal{R}_{J} \approx \Sigma_{J}\left(J^{s t}\right)
\end{aligned}
$$

and the stabilised strains are evaluated as

$$
\begin{array}{rlrl}
\boldsymbol{F}^{s t} & =\boldsymbol{F}-\tau_{\boldsymbol{F}} \boldsymbol{\mathcal { R }}_{\boldsymbol{F}} ; & \mathcal{R}_{\boldsymbol{F}}=\dot{\boldsymbol{F}}-\boldsymbol{\nabla}_{0} \boldsymbol{v} \\
\boldsymbol{H}^{s t}=\boldsymbol{H}-\tau_{\boldsymbol{H}} \boldsymbol{\mathcal { R }}_{\boldsymbol{H}} ; & \mathcal{R}_{\boldsymbol{H}}=\dot{\boldsymbol{H}}-\boldsymbol{F} \times \boldsymbol{\nabla}_{0} \boldsymbol{v} \\
J^{s t}=J-\tau_{J} \mathcal{R}_{J} ; & & \mathcal{R}_{J}=\dot{J}-\boldsymbol{H}: \boldsymbol{\nabla}_{0} \boldsymbol{v} .
\end{array}
$$

Note that the units of the four $\tau$-parameters (i.e. $\tau_{\boldsymbol{v}}, \tau_{\boldsymbol{F}}, \tau_{\boldsymbol{H}}$ and $\tau_{J}$ ) are those of time and are usually chosen as a fraction of the time step for explicit integration schemes [73, 94, 95, 101].

In the above three equations (100), the residual terms represent the difference between the time rate of the corresponding strain variable and its evaluation in terms of the gradient of velocities. Given that these time rates appear in the left hand side of the system of equations, it is not possible to derive a completely explicit scheme. In order to rectify this, a simple procedure has been proposed in references $[1,8]$ whereby the above time residuals are replaced by their time integrated geometric equivalents to give stabilised strains as:

$$
\begin{array}{rlrl}
\boldsymbol{F}^{s t} & =\boldsymbol{F}-\tau_{\boldsymbol{F}} \boldsymbol{\mathcal { R }}_{\boldsymbol{F}}-\zeta_{\boldsymbol{F}} \boldsymbol{\mathcal { R }}_{\boldsymbol{F}}^{\boldsymbol{x}} ; & \mathcal{R}_{\boldsymbol{F}}^{\boldsymbol{x}}=\boldsymbol{F}-\boldsymbol{F}_{\boldsymbol{x}} ; & \boldsymbol{F}_{\boldsymbol{x}}=\nabla_{0} \boldsymbol{x} \\
\boldsymbol{H}^{s t}=\boldsymbol{H}-\tau_{\boldsymbol{H}} \boldsymbol{\mathcal { R }}_{\boldsymbol{H}}-\zeta_{\boldsymbol{H}} \boldsymbol{\mathcal { R }}_{\boldsymbol{H}}^{\boldsymbol{x}} ; & \mathcal{R}_{\boldsymbol{H}}^{\boldsymbol{x}}=\boldsymbol{H}-\boldsymbol{H}_{\boldsymbol{x}} ; & \boldsymbol{H}_{\boldsymbol{x}}=\frac{1}{2} \boldsymbol{\nabla}_{0} \boldsymbol{x} \times \boldsymbol{\nabla}_{0} \boldsymbol{x} \\
J^{s t} & =J-\tau_{J} \mathcal{R}_{J}-\zeta_{J} \mathcal{R}_{J}^{\boldsymbol{x}} ; & \mathcal{R}_{J}^{\boldsymbol{x}}=J-J_{\boldsymbol{x}} ; & J_{\boldsymbol{x}}=\operatorname{det} \boldsymbol{\nabla}_{0} \boldsymbol{x} .
\end{array}
$$

where $\zeta_{\boldsymbol{F}}, \zeta_{\boldsymbol{H}}$ and $\zeta_{J}$ are dimensionless stabilisation parameters. Introducing the linear interpolations for the momentum and virtual velocity fields gives 
a set of equations for the rate of change of momentum at each node $a$ given as:

$$
\sum_{b} M_{a b} \dot{\boldsymbol{p}}_{b}=\int_{V} \boldsymbol{f}_{0} N_{a} d V+\int_{\partial_{t} V} \boldsymbol{t}_{B} N_{a} d A-\int_{V} \boldsymbol{P}^{s t} \nabla_{0} N_{a} d V
$$

where the consistent mass contribution $M_{a b}=\int_{V} N_{a} N_{b} d V$.

Remark 2: $\quad$ Note that choosing $\tau_{\boldsymbol{F}}=\tau_{\boldsymbol{H}}=\tau_{J}=0$ and $\zeta_{\boldsymbol{F}}=\zeta_{\boldsymbol{H}}=\zeta_{J}=1$ will lead to a displacement based formulation as the strain measures are simply replaced by those derived from the element geometry.

Finally, the stabilised conjugate stress measures defined in equation (96) can be introduced into the weighted residual equation (88) and, after following a similar integration by parts procedure to that shown in equation (91), gives a stabilised set of strain update equations as:

$$
\begin{aligned}
\int_{V} \delta \boldsymbol{\Sigma}_{\boldsymbol{F}}: \frac{\partial \boldsymbol{F}}{\partial t} d V & =-\int_{V} \frac{1}{\rho_{0}} \boldsymbol{p}^{s t} \cdot \mathbf{D I V}\left(\delta \boldsymbol{\Sigma}_{\boldsymbol{F}}\right) d V+\int_{\partial_{u} V} \boldsymbol{v}_{B} \cdot \delta \boldsymbol{\Sigma}_{\boldsymbol{F}} \boldsymbol{N} d A \\
\int_{V} \delta \boldsymbol{\Sigma}_{\boldsymbol{H}}: \frac{\partial \boldsymbol{H}}{\partial t} d V & =\int_{V}\left(\frac{1}{\rho_{0}} \boldsymbol{p}^{s t} \mathbf{x} \boldsymbol{F}\right): \mathbf{C U R L}\left(\delta \boldsymbol{\Sigma}_{\boldsymbol{H}}\right) d V-\int_{\partial_{u} V}\left(\boldsymbol{v}_{B} \times \boldsymbol{F} \times \boldsymbol{N}\right): \delta \boldsymbol{\Sigma}_{\boldsymbol{H}} d A \\
\int_{V} \delta \Sigma_{J} \frac{\partial J}{\partial t} d V & =-\int_{V} \frac{1}{\rho_{0}} \boldsymbol{p}^{s t} \cdot \boldsymbol{H} \nabla_{0}\left(\delta \Sigma_{J}\right) d V+\int_{\partial_{u} V}\left(\boldsymbol{v}_{B} \cdot \boldsymbol{H} \boldsymbol{N}\right) \delta \Sigma_{J} d A
\end{aligned}
$$

where the stabilised linear momentum is defined by:

$$
\boldsymbol{p}^{s t}=\boldsymbol{p}-\tau_{\boldsymbol{v}} \boldsymbol{\mathcal { R }}_{\boldsymbol{p}}
$$

However, in applying this correction to the linear momentum $\boldsymbol{p}$, the deformation gradient $\boldsymbol{F}$ will no longer be a discrete gradient (in some weighted residual sense) of a continuous velocity field, since the above residual term will be discontinuous across the element edges. Similarly, the co-factor matrix $\boldsymbol{H}$ will no longer be the curl of a continuous function. This implies that the involutions described by equation (6) are no longer enforced. For this reason, the stabilisation of the momentum field should only be applied to the third equation above for $J$. Finally, introducing the linear interpolation equations for the strain variables and their conjugate stresses gives a set of 
equations for the nodal strain values as:

$$
\begin{aligned}
\sum_{b} M_{a b} \dot{\boldsymbol{F}}_{b} & =-\int_{V} \frac{1}{\rho_{0}} \boldsymbol{p} \otimes \nabla_{0} N_{a} d V+\int_{\partial_{u} V} N_{a} \boldsymbol{v}_{B} \otimes \boldsymbol{N} d A \\
\sum_{b} M_{a b} \dot{\boldsymbol{H}}_{b} & =\int_{V} \frac{1}{\rho_{0}} \boldsymbol{p} \times \boldsymbol{F} \times \nabla_{0} N_{a} d V-\int_{\partial_{u} V} N_{a} \boldsymbol{v}_{B} \times \boldsymbol{F} \times \boldsymbol{N} d A \\
\sum_{b} M_{a b} \dot{J}_{b} & =-\int_{V} \frac{1}{\rho_{0}} \boldsymbol{p}^{s t} \cdot \boldsymbol{H} \nabla_{0} N_{a} d V+\int_{\partial_{u} V}\left(\boldsymbol{v}_{B} \cdot \boldsymbol{H} \boldsymbol{N}\right) N_{a} d A
\end{aligned}
$$

The set of equations (102) and (105) represents a stabilised system of differential equations in time for the conservation variables $\{\boldsymbol{p}, \boldsymbol{F}, \boldsymbol{H}, J\}$. Choosing $\tau_{J}=0$ and appropriate non-dimensional values of $\left\{\zeta_{\boldsymbol{F}}, \zeta_{\boldsymbol{H}}, \zeta_{J}\right\}$, typically in the range of $[0,0.5]$, it is possible to derive a completely explicit integration scheme. Moreover, the use of a lumped mass matrix instead of the consistent mass matrix does not alter the order of convergence of the algorithm $[1,8]$.

Insofar as the deformation gradient $\boldsymbol{F}$ is not computed from the gradient of the current geometry $(1)_{1}$, the computational algorithm presented thus far does not necessarily preserve angular momentum. To rectify this, the authors already introduced a correction into the space-time integrator to ensure the preservation of angular momentum. Detailed explanation can be found in section 4.1 in reference [8].

Remark 3: The stabilisation process described above in terms of entropy conjugate variables leads to identical additional terms to those obtained with a residual based variational multi-scale procedure [72, 102].

\subsection{SUPG stabilisation using entropy variables}

In this case, the residual of the conservation laws is formulated with respect to the conjugate entropy variables $\mathcal{R}(\mathcal{V})(87)$. The Galerkin weighted residual equations described in (88) require stabilisation, introduced by means of a Petrov-Galerkin approach whereby the conjugate weighting functions $\delta \mathcal{V}$ are augmented as:

$$
\delta \mathcal{V}^{s t}=\delta \mathcal{V}+\tilde{\boldsymbol{\tau}} \tilde{\mathcal{A}}_{I} \frac{\partial \delta \mathcal{V}}{\partial X_{I}}
$$


where $\tilde{\boldsymbol{\tau}}$ denotes a symmetric matrix of stabilisation parameters. An elegant procedure to determine the symmetric stabilisation matrix $\tilde{\boldsymbol{\tau}}$ was introduced in [72] and it is summarised here for completeness for the one and two dimensional scenarios.

\subsubsection{One dimensional symmetric equations for elastodynamics}

For ease of understanding, the stabilisation process presented in above section 5.4 is particularised for the one dimensional case. In the case of the linearised elastodynamics problem [50], system (66) reduces to:

$$
\begin{aligned}
& \rho_{0} \frac{\partial v_{x}}{\partial t}-\frac{\partial \Sigma_{F_{x X}}}{\partial X}=f_{0_{x}} \\
& \frac{1}{E} \frac{\partial \Sigma_{F_{x X}}}{\partial t}-\frac{\partial v_{x}}{\partial X}=0
\end{aligned}
$$

where the conjugate stress $\Sigma_{F_{x X}}$ is related to the deformation gradient $F_{x X}$ via the Young's modulus $E$ (i.e. $\Sigma_{F_{x X}}=E\left(F_{x X}-1\right)$ ). The symmetric matrices $\mathcal{A}_{0}$ and $\tilde{\mathcal{A}}_{X}$ can be written as (refer to equation (67)):

$$
\mathcal{A}_{0}=\left[\begin{array}{cc}
\rho_{0} & 0 \\
0 & \frac{1}{E}
\end{array}\right] ; \quad \tilde{\mathcal{A}}_{X}=\left[\begin{array}{cc}
0 & -1 \\
-1 & 0
\end{array}\right]
$$

Definition of the matrix $\mathcal{L}=\mathcal{A}_{0}^{1 / 2}$ and $\hat{\mathcal{A}_{X}}=\mathcal{L}^{-1} \tilde{\mathcal{A}}_{X} \mathcal{L}^{-T}$ yields

$$
\hat{\mathcal{A}}_{X}=\left[\begin{array}{cc}
0 & -c_{p} \\
-c_{p} & 0
\end{array}\right] ; \quad c_{p}=\sqrt{\frac{E}{\rho_{0}}}
$$

with eigen-decomposition $\hat{\mathcal{A}}_{X}=\sum_{\alpha=1}^{2} c_{\alpha}\left(\overline{\mathcal{W}}_{\alpha} \otimes \overline{\mathcal{W}}_{\alpha}\right)$ with

$$
c_{1,2}= \pm c_{p} ; \quad \overline{\mathcal{W}}_{1}=\left[\begin{array}{c}
-\frac{1}{\sqrt{2}} \\
\frac{1}{\sqrt{2}}
\end{array}\right] ; \quad \overline{\mathcal{W}}_{2}=\left[\begin{array}{c}
\frac{1}{\sqrt{2}} \\
\frac{1}{\sqrt{2}}
\end{array}\right]
$$

The matrix of stabilisation parameters $\hat{\boldsymbol{\tau}}$ is defined as $\hat{\boldsymbol{\tau}}=\frac{h}{2}\left|\hat{\mathcal{A}_{X}}\right|^{-1}$ which can be obtained as

$$
\hat{\boldsymbol{\tau}}=\frac{h}{2} \sum_{\alpha=1}^{2} \frac{1}{\left|c_{\alpha}\right|}\left(\overline{\mathcal{W}}_{\alpha} \otimes \overline{\mathcal{W}}_{\alpha}\right)=\frac{h}{2 c_{p}}\left[\begin{array}{ll}
1 & 0 \\
0 & 1
\end{array}\right] .
$$

In above expression (111), $\frac{h}{2}$ denotes the deformation gradient from the reference domain to the isoparametric domain, being $h$ the size of the finite 
element under consideration. Finally, the matrix of stabilisation parameters $\tilde{\tau}$ is obtained as:

$$
\tilde{\boldsymbol{\tau}}=\mathcal{L}^{-T} \hat{\boldsymbol{\tau}} \mathcal{L}^{-1}=\frac{h}{2 c_{p}}\left[\begin{array}{cc}
\frac{1}{\rho_{0}} & 0 \\
0 & E
\end{array}\right] .
$$

The stabilised Petrov-Galerkin variational statement for the one dimensional elastodynamics problem defined in entropy variables is:

$$
\int_{V} \underbrace{\left(\delta \mathcal{V}+\tilde{\boldsymbol{\tau}} \tilde{\mathcal{A}}_{X} \frac{\partial \delta \mathcal{V}}{\partial X}\right)^{T}}_{\delta \mathcal{V}^{s t}} \mathcal{R} d V=0
$$

where

$$
\delta \mathcal{V}^{s t}=\left[\begin{array}{c}
\delta v_{x}-\frac{h}{2} \frac{1}{c_{p} \rho_{0}} \frac{\partial \delta \Sigma_{F_{X X}}}{\partial X} \\
\delta \Sigma_{F_{x X}}-\frac{h}{2} \frac{E}{c_{p}} \frac{\partial \delta v_{x}}{\partial X}
\end{array}\right] ; \quad \mathcal{R}=\left[\begin{array}{c}
\frac{\partial \Sigma_{F_{X X}}}{\partial X}+f_{0_{x}}-\rho_{0} \frac{\partial v_{x}}{\partial t} \\
\frac{\partial v_{x}}{\partial X}-\frac{1}{E} \frac{\partial \Sigma_{F_{x X}}}{\partial t}
\end{array}\right] .
$$

Substitution of formulae (114) into (113) accompanied by integration by parts and the gathering of terms according to each virtual conjugate variable, leads to:

$$
\begin{aligned}
\int_{V} \delta v_{x}\left(\rho_{0} \frac{\partial v_{x}}{\partial t}\right) d V & =\int_{\partial_{t} V} \delta v_{x} t_{B_{x}} d A+\int_{V} \delta v_{X} f_{0_{x}} d V-\int_{V} \Sigma_{F_{x X}}^{s t} \frac{\partial \delta v_{x}}{\partial X} d V \\
\int_{V} \delta \Sigma_{F_{x X}}\left(\frac{1}{E} \frac{\partial \Sigma_{F_{x X}}}{\partial t}\right) d V & =\int_{\partial_{u} V} \delta \Sigma_{F_{x X}} v_{B_{x}} d A-\int_{V} v_{x}^{s t} \frac{\partial \delta \Sigma_{F_{x X}}}{\partial X} d V .
\end{aligned}
$$

where $t_{B_{x}}$ and $v_{B_{x}}$ represent prescribed boundary conditions. In this particular case, the use of integration by parts in the above expressions does not affect the symmetry of the system. The corresponding stabilised stress $\Sigma_{F_{x X}}^{s t}$ and velocity $v_{x}^{s t}$ can be written as:

$$
\begin{aligned}
\Sigma_{F_{x X}}^{s t} & =\Sigma_{F_{x X}}+\tau_{F} E\left(\frac{\partial v_{x}}{\partial X}-\frac{1}{E} \frac{\partial \Sigma_{F_{x X}}}{\partial t}\right) \\
v_{x}^{s t} & =v_{x}+\frac{\tau_{v}}{\rho_{0}}\left(\frac{\partial \Sigma_{F_{x X}}}{\partial X}-\rho_{0} \frac{\partial v_{x}}{\partial t}\right),
\end{aligned}
$$

where $\tau_{F}=\tau_{v}=\frac{h}{2 c_{p}}$. Note that the stabilised stress $\Sigma_{F_{x X}}^{s t}$ (116a) can be equivalently interpreted as the conjugate stress of a stabilised deformation gradient defined by

$$
\Sigma_{F_{x X}}^{s t}=E\left(F_{x X}^{s t}-1\right) ; \quad F_{x X}^{s t}=F_{x X}+\tau_{F}\left(\frac{\partial v_{x}}{\partial X}-\frac{1}{E} \frac{\partial \Sigma_{F_{x X}}}{\partial t}\right),
$$


which is widely known as Variational Multi-Scale (VMS) method [73, 82, 83, 85-90]. As can be observed, in this case the formulation in entropy variables renders an identical result to that obtained using the conservation variables.

\subsubsection{Two dimensional symmetric equations for elastodynamics}

The two dimensional equations for the elastodynamics problem formulated in entropy variables and for a compressible Neo-Hookean model (refer to equations (20) and (22)) can be deduced from (66) as:

$$
\begin{aligned}
\rho_{0} \frac{\partial \boldsymbol{v}}{\partial t}-\operatorname{DIV}_{\boldsymbol{F}}-\boldsymbol{H}_{\boldsymbol{\Sigma}_{\boldsymbol{F}}} \boldsymbol{\nabla}_{0} \Sigma_{J} & =\boldsymbol{f}_{0} \\
\frac{1}{\mu} \frac{\partial \boldsymbol{\Sigma}_{\boldsymbol{F}}}{\partial t}-\nabla_{0} \boldsymbol{v} & =\mathbf{0} \\
\frac{1}{\gamma} \frac{\partial \Sigma_{J}}{\partial t}-\boldsymbol{H}_{\boldsymbol{\Sigma}_{F}}: \boldsymbol{\nabla}_{0} \boldsymbol{v} & =0
\end{aligned}
$$

where the co-factor $\boldsymbol{H}_{\boldsymbol{\Sigma}_{\boldsymbol{F}}}$ is obtained in terms of the conjugate stress to the deformation gradient $\boldsymbol{H}_{\boldsymbol{\Sigma}_{\boldsymbol{F}}}=\boldsymbol{H}\left(\boldsymbol{\Sigma}_{\boldsymbol{F}}\right)$ as

$$
\boldsymbol{H}_{\boldsymbol{\Sigma}_{\boldsymbol{F}}}=\frac{1}{\mu}\left[\begin{array}{rr}
\Sigma_{F_{y Y}} & -\Sigma_{F_{y X}} \\
-\Sigma_{F_{x Y}} & \Sigma_{F_{x X}}
\end{array}\right]
$$

Notice that in a two dimensional problem, the co-factor $\boldsymbol{H}$ can be directly

obtained from the deformation gradient $\boldsymbol{F}$ (or its conjugate stress $\boldsymbol{\Sigma}_{\boldsymbol{F}}$ ) and hence, the evolution equation for the co-factor is clearly redundant. The quasilinear form of the system (118) can be written as:

$$
\mathcal{A}_{0} \frac{\partial \mathcal{V}}{\partial t}+\tilde{\mathcal{A}}_{X} \frac{\partial \mathcal{V}}{\partial X}+\tilde{\mathcal{A}}_{Y} \frac{\partial \mathcal{V}}{\partial Y}=\mathcal{S}
$$

where the components of the entropy variables $\mathcal{V}$, the source term $\mathcal{S}$ and the matrix $\mathcal{A}_{0}$ are:

$$
\mathcal{V}=\left[\begin{array}{c}
v_{x} \\
v_{y} \\
\Sigma_{F_{x X}} \\
\Sigma_{F_{x Y}} \\
\Sigma_{F_{y X}} \\
\Sigma_{F_{y Y}} \\
\Sigma_{J}
\end{array}\right] ; \quad \mathcal{S}=\left[\begin{array}{c}
f_{0_{x}} \\
f_{0_{y}} \\
0 \\
0 \\
0 \\
0 \\
0
\end{array}\right] ; \quad \mathcal{A}_{0}=\left[\begin{array}{ccccccc}
\rho_{0} & 0 & 0 & 0 & 0 & 0 & 0 \\
0 & \rho_{0} & 0 & 0 & 0 & 0 & 0 \\
0 & 0 & \frac{1}{\mu} & 0 & 0 & 0 & 0 \\
0 & 0 & 0 & \frac{1}{\mu} & 0 & 0 & 0 \\
0 & 0 & 0 & 0 & \frac{1}{\mu} & 0 & 0 \\
0 & 0 & 0 & 0 & 0 & \frac{1}{\mu} & 0 \\
0 & 0 & 0 & 0 & 0 & 0 & \frac{1}{\gamma}
\end{array}\right]
$$


and the corresponding symmetric flux Jacobian matrices in $O X$ and $O Y$ directions are:

$$
\begin{aligned}
& \tilde{\mathcal{A}_{X}}=\left[\begin{array}{rrrrrrr}
0 & 0 & -1 & 0 & 0 & 0 & -\frac{1}{\mu} \Sigma_{F_{y Y}} \\
0 & 0 & 0 & 0 & -1 & 0 & \frac{1}{\mu} \Sigma_{F_{x Y}} \\
-1 & 0 & 0 & 0 & 0 & 0 & 0 \\
0 & 0 & 0 & 0 & 0 & 0 & 0 \\
0 & -1 & 0 & 0 & 0 & 0 & 0 \\
0 & 0 & 0 & 0 & 0 & 0 & 0 \\
-\frac{1}{\mu} \Sigma_{F_{y Y}} & \frac{1}{\mu} \Sigma_{F_{x Y}} & 0 & 0 & 0 & 0 & 0
\end{array}\right] \\
& \tilde{\mathcal{A}}_{Y}=\left[\begin{array}{rrrrrrr}
0 & 0 & 0 & -1 & 0 & 0 & \frac{1}{\mu} \Sigma_{F_{y X}} \\
0 & 0 & 0 & 0 & 0 & -1 & -\frac{1}{\mu} \Sigma_{F_{x X}} \\
0 & 0 & 0 & 0 & 0 & 0 & 0 \\
-1 & 0 & 0 & 0 & 0 & 0 & 0 \\
0 & 0 & 0 & 0 & 0 & 0 & 0 \\
0 & -1 & 0 & 0 & 0 & 0 & 0 \\
\frac{1}{\mu} \Sigma_{F_{y X}} & -\frac{1}{\mu} \Sigma_{F_{x X}} & 0 & 0 & 0 & 0 & 0
\end{array}\right] \text {. }
\end{aligned}
$$

After definition of the matrix $\mathcal{L}=\mathcal{A}_{0}^{1 / 2}$, the eigen-system for each of the flux Jacobian matrices $\hat{\hat{\mathcal{A}}_{X}}=\mathcal{L}^{-1} \tilde{\mathcal{A}}_{X} \mathcal{L}^{-T}$ and $\hat{\mathcal{A}_{Y}}=\mathcal{L}^{-1} \tilde{\mathcal{A}}_{Y} \mathcal{L}^{-T}$ can be solved as a special case of that presented in (74) and thereafter. Specifically, the eigenvalues are

$$
\begin{array}{ll}
c_{1,2}^{X}= \pm\left(\sqrt{\frac{\mu+\gamma / \mu\left[\left(\Sigma_{F_{x Y}}\right)^{2}+\left(\Sigma_{F_{y Y}}\right)^{2}\right]}{\rho_{0}}}\right) ; & c_{3,4}^{X}= \pm \sqrt{\frac{\mu}{\rho_{0}}} \\
c_{1,2}^{Y}= \pm\left(\sqrt{\frac{\mu+\gamma / \mu\left[\left(\Sigma_{F_{x X}}\right)^{2}+\left(\Sigma_{F_{y X}}\right)^{2}\right]}{\rho_{0}}}\right) ; & c_{3,4}^{Y}= \pm \sqrt{\frac{\mu}{\rho_{0}}}
\end{array}
$$


and the eigenvectors are

$$
\begin{aligned}
& \overline{\mathcal{W}}_{1,2}^{X}=\left[\begin{array}{c}
\sqrt{\rho_{0}} \frac{\Sigma_{F_{y Y}}}{D_{X}} \\
-\sqrt{\rho_{0}} \frac{\Sigma_{F_{X Y}}}{D_{X}} \\
-\frac{\sqrt{\mu}}{c_{1,2}^{X}} \frac{\Sigma_{F_{y Y}}}{D_{X}} \\
0 \\
\frac{\sqrt{\mu}}{c_{1,2}^{X}} \frac{\Sigma_{F_{x Y}}}{D_{X}} \\
0 \\
-\frac{\sqrt{\gamma}}{c_{1,2}^{X} \mu} D_{X}
\end{array}\right] ; \quad \overline{\mathcal{W}}_{3,4}^{X}=\left[\begin{array}{c}
\sqrt{\rho_{0}} \frac{\Sigma_{F_{x Y}}}{D_{X}} \\
\sqrt{\rho_{0}} \frac{\Sigma_{F_{y} Y}}{D_{X}} \\
-\frac{\sqrt{\mu}}{c_{3,4}^{X}} \frac{\Sigma_{F_{x Y}}}{D_{X}} \\
0 \\
-\frac{\sqrt{\mu}}{c_{3,4}^{X}} \frac{\Sigma_{F_{y Y}}}{D_{X}} \\
0 \\
0
\end{array}\right] ; \quad D_{X}=\left(\Sigma_{F_{x Y}}^{2}+\Sigma_{F_{y Y}}^{2}\right)^{1 / 2} \\
& \overline{\mathcal{W}}_{1,2}^{Y}=\left[\begin{array}{c}
-\sqrt{\rho_{0}} \frac{\Sigma_{F_{y X}}}{D_{Y}} \\
\sqrt{\rho_{0}} \frac{\Sigma_{F_{x X}}}{D_{Y}} \\
0 \\
\frac{\sqrt{\mu}}{c_{1,2}^{Y}} \frac{\Sigma_{F_{y X}}}{D_{Y}} \\
0 \\
-\frac{\sqrt{\mu}}{c_{1,2}^{Y}} \frac{\Sigma_{F_{x X}}}{D_{Y}} \\
-\frac{\sqrt{\gamma}}{c_{1,2}^{Y} \mu} D_{Y}
\end{array}\right] ; \quad \overline{\mathcal{W}}_{3,4}^{Y}=\left[\begin{array}{c}
\sqrt{\rho_{0}} \frac{\Sigma_{F_{x X}}}{D_{Y}} \\
\sqrt{\rho_{0}} \frac{\Sigma_{F_{y X}}}{D_{Y}} \\
0 \\
-\frac{\sqrt{\mu}}{c_{3,4}^{Y}} \frac{\Sigma_{F_{x X}}}{D_{Y}} \\
0 \\
-\frac{\sqrt{\mu}}{c_{3,4}^{Y}} \frac{\Sigma_{F_{y X}}}{D_{Y}} \\
0
\end{array}\right] ; \quad D_{Y}=\left(\Sigma_{F_{x X}}^{2}+\Sigma_{F_{y X}}^{2}\right)^{1 / 2} .
\end{aligned}
$$

Reference [72] generalises the one dimensional concept presented in the previous section for the multi-dimensional case. For this scenario, the matrix of stabilisation parameters $\hat{\boldsymbol{\tau}}$ is defined in terms of the inverse of the $p$-norm of the flux Jacobian operator $\mathcal{A}$ as $|\hat{\mathcal{A}}|_{p}$, namely $\hat{\boldsymbol{\tau}}=\frac{h}{2}|\hat{\mathcal{A}}|_{p}^{-1}$, defined ${ }^{5}$ in terms of its spatial components as follows

$$
|\hat{\mathcal{A}}|_{p}^{-1}=\left(\left|\hat{\mathcal{A}_{X}}\right|^{p}+\left|\hat{\mathcal{A}}_{Y}\right|^{p}\right)^{-1 / p}
$$

where

$$
\left|\hat{\mathcal{A}_{X}}\right|^{p}=\sum_{\alpha=1}^{4}\left|c_{\alpha}^{X}\right|^{p}\left(\overline{\mathcal{W}}_{\alpha}^{X} \otimes \overline{\mathcal{W}}_{\alpha}^{X}\right) ; \quad\left|\hat{\mathcal{A}}_{Y}\right|^{p}=\sum_{\alpha=1}^{4}\left|c_{\alpha}^{Y}\right|^{p}\left(\overline{\mathcal{W}}_{\alpha}^{Y} \otimes \overline{\mathcal{W}}_{\alpha}^{Y}\right)
$$

\footnotetext{
${ }^{5}$ In this case, $\frac{h}{2} \boldsymbol{I}$ denotes the deformation gradient from the reference domain to the isoparametric domain, being $h$ the size of the finite element under consideration. The methodology can be extended to other finite element discretisations [72].
} 
Notice that in the case of an odd norm (e.g. $p=2 k+1, k \in \mathbb{N}$ ), the use of the spectral decomposition theorem is necessary when evaluating expressions in $(126)^{6}$. The evaluation of expression (125) must also be carried out via the spectral decomposition theorem as

$$
|\hat{\mathcal{A}}|_{p}^{-1}=\sum_{\alpha=1}^{n_{\alpha}} \frac{1}{\left(a_{\alpha}\right)^{p}}\left(\overline{\mathcal{X}}_{\alpha} \otimes \overline{\mathcal{X}}_{\alpha}\right)
$$

where $a_{\alpha}$ and $\overline{\boldsymbol{\mathcal { X }}}_{\alpha}$ are the $n_{\alpha}$ (in general, $n_{\alpha}>4$ ) eigenvalues and eigenvectors associated to the following eigen-system

$$
\left(\left|\hat{\mathcal{A}}_{X}\right|^{p}+\left|\hat{\mathcal{A}}_{Y}\right|^{p}\right) \overline{\mathcal{X}}_{\alpha}=a_{\alpha} \overline{\mathcal{X}}_{\alpha}
$$

Finally, the matrix of stabilisation parameters $\tilde{\boldsymbol{\tau}}$ is obtained as $\tilde{\boldsymbol{\tau}}=$ $\mathcal{L}^{-T} \hat{\boldsymbol{\tau}} \mathcal{L}^{-1}$. The stabilised Petrov-Galerkin variational statement for the two dimensional elastodynamics problem is:

$$
\int_{V} \underbrace{\left[\delta \mathcal{V}+\tilde{\boldsymbol{\tau}}\left(\tilde{\mathcal{A}_{X}} \frac{\partial \delta \mathcal{V}}{\partial X}+\tilde{\mathcal{A}}_{Y} \frac{\partial \delta \mathcal{V}}{\partial Y}\right)\right]^{T}}_{\delta \mathcal{V}^{\text {st }}} \mathcal{R} d V=0
$$

with terms $\tilde{\boldsymbol{\tau}}, \tilde{\mathcal{A}_{X}}$ and $\tilde{\mathcal{A}_{Y}}$ as defined above. In the case of using $p=2$ (as in the examples shown later in the paper) when evaluating (125), it is possible to show $[72,103]$ that a simplified expression for the stabilisation parameter $\tilde{\tau}$ can be obtained as follows:

$$
\tilde{\boldsymbol{\tau}}=\frac{h}{2} \mathcal{A}_{0}^{-1}\left[\left(\tilde{\mathcal{A}_{X}} \mathcal{A}_{0}^{-1}\right)^{2}+\left(\tilde{\mathcal{A}_{Y}} \mathcal{A}_{0}^{-1}\right)^{2}\right]^{-1 / 2} .
$$

To reduce the level of implicitness of the coupled formulation (see equation (129)) and, more importantly, to ensure the satisfaction of the involutions $(6)_{2}$ in a weighted residual sense (129), the components of the stabilisation matrix $\tilde{\boldsymbol{\tau}}$ corresponding to the conjugate stress $(118)_{2}$ are neglected. This leads to a robust and relatively fast algorithm.

\footnotetext{
${ }^{6}$ For even norms, $p=2 k, k \in \mathbb{N}$, this can be avoided as $\left|\tilde{\mathcal{A}}_{I}\right|^{p}=\left(\tilde{\mathcal{A}}_{I}\right)^{p}$.
} 
Regardless of the procedure followed to obtain $\tilde{\boldsymbol{\tau}}$, the resulting stabilisation matrix $\tilde{\boldsymbol{\tau}}$ is a non-sparse matrix in general. However, let us assume, for simplicity, a diagonal structure for this matrix $\tilde{\boldsymbol{\tau}}$ as follows

$$
\tilde{\boldsymbol{\tau}}=\left[\begin{array}{ccccccc}
\frac{1}{\rho_{0}} \tau_{\boldsymbol{v}} & 0 & 0 & 0 & 0 & 0 & 0 \\
0 & \frac{1}{\rho_{0}} \tau_{\boldsymbol{v}} & 0 & 0 & 0 & 0 & 0 \\
0 & 0 & \mu \tau_{\boldsymbol{F}} & 0 & 0 & 0 & 0 \\
0 & 0 & 0 & \mu \tau_{\boldsymbol{F}} & 0 & 0 & 0 \\
0 & 0 & 0 & 0 & \mu \tau_{\boldsymbol{F}} & 0 & 0 \\
0 & 0 & 0 & 0 & 0 & \mu \tau_{\boldsymbol{F}} & 0 \\
0 & 0 & 0 & 0 & 0 & 0 & \gamma \tau_{J}
\end{array}\right]
$$

In this case, the resulting virtual stabilised entropy conjugate variables $\delta \mathcal{V}^{s t}$ reduce to:

$$
\begin{aligned}
\delta \boldsymbol{v}^{s t} & =\delta \boldsymbol{v}-\frac{\tau_{\boldsymbol{v}}}{\rho_{0}}\left(\mathbf{D I V} \delta \boldsymbol{\Sigma}_{\boldsymbol{F}}+\boldsymbol{H}_{\boldsymbol{\Sigma}_{\boldsymbol{F}}} \boldsymbol{\nabla}_{0} \delta \Sigma_{J}\right) \\
\delta \boldsymbol{\Sigma}_{\boldsymbol{F}}{ }^{s t} & =\delta \boldsymbol{\Sigma}_{\boldsymbol{F}}-\tau_{\boldsymbol{F}} \mu\left(\boldsymbol{\nabla}_{0} \delta \boldsymbol{v}\right) \\
\delta \Sigma_{J}^{s t} & =\delta \Sigma_{J}-\tau_{J} \gamma\left(\boldsymbol{H}_{\boldsymbol{\Sigma}_{\boldsymbol{F}}}: \boldsymbol{\nabla}_{0} \delta \boldsymbol{v}\right)
\end{aligned}
$$

where it is easy to see that, in this particular case, the stabilised virtual conjugate variables $\delta \mathcal{V}^{s t}$ coincide with that of equation (96) when using conservation variables.

Irrespective of using system (66) or (68), and in order to preserve the symmetry of the system, the Gauss theorem must not be employed in the linear momentum balance principle. This implies that traction boundary conditions on $\partial_{t} V$, usually applied via Neumann boundary conditions when using conservative variables (90), must be imposed in an alternative manner.

In this paper, a simple Dirichlet-based Lagrange multiplier projection procedure is employed where the value of the conjugate stresses $\left(\boldsymbol{\Sigma}_{\boldsymbol{F}}\right.$ and $\Sigma_{J}$ ) on the boundary of the continuum is projected via the minimisation of a functional $\Pi$ defined as follows ${ }^{7}$,

$$
\Pi\left(\boldsymbol{\Sigma}_{\boldsymbol{F}}^{*}, \Sigma_{J}^{*}, \boldsymbol{\lambda}\right)=\frac{1}{2}\left(\boldsymbol{\Sigma}_{\boldsymbol{F}}^{*}-\boldsymbol{\Sigma}_{\boldsymbol{F}}\right):\left(\boldsymbol{\Sigma}_{\boldsymbol{F}}^{*}-\boldsymbol{\Sigma}_{\boldsymbol{F}}\right)+\frac{1}{2}\left(\Sigma_{J}^{*}-\Sigma_{J}\right)^{2}+\boldsymbol{\lambda} \cdot\left(\boldsymbol{t}_{B}-\boldsymbol{P}^{*} \boldsymbol{N}\right)
$$

\footnotetext{
${ }^{7}$ For simplicity, the procedure is presented for the case of a compressible Neo-Hookean constitutive model, where the conjugate stress $\boldsymbol{\Sigma}_{\boldsymbol{H}}$ is not required.
} 
where $\boldsymbol{t}_{B}$ is the prescribed boundary traction vector on $\partial_{t} V, \Sigma_{\boldsymbol{F}}^{*}$ and $\Sigma_{J}^{*}$ are the projected conjugate stresses leading to a projected boundary PiolaKirchhoff stress defined as $\boldsymbol{P}^{*}=\boldsymbol{\Sigma}_{\boldsymbol{F}}^{*}+\Sigma_{J}^{*} \boldsymbol{H}_{\boldsymbol{\Sigma}_{\boldsymbol{F}}}$ and $\boldsymbol{\lambda}$ denotes a Lagrange multiplier vector.

This projection procedure is applied locally at all the degrees of freedom placed on $\partial_{t} V$. Moreover, the last term on the right hand side of equation (133) must be split into as many facets as those connected to the relevant degree of freedom. Notice that the co-factor in the second term of the projected Piola-Kirchhoff stress $\boldsymbol{P}^{*}$ is defined as $\boldsymbol{H}_{\boldsymbol{\Sigma}_{F}}$, and not $\boldsymbol{H}_{\boldsymbol{\Sigma}_{F}^{*}}$, in order to avoid the resolution of a nonlinear system of equations when applying this projection procedure. A more general nonlinear projection procedure, if needed, can be derived without further difficulty.

The stationary condition of the above functional (133) with respect to $\boldsymbol{\lambda}$ and the corrected conjugate stresses $\left\{\Sigma_{\boldsymbol{F}}^{*}, \Sigma_{J}^{*}\right\}$ are considered separately. Note firstly that the directional derivative of $\Pi$ with respect to a variation along $\boldsymbol{\lambda}$ leads to the constraint

$$
\boldsymbol{t}_{B}-\left(\boldsymbol{\Sigma}_{\boldsymbol{F}}^{*}+\Sigma_{J}^{*} \boldsymbol{H}_{\boldsymbol{\Sigma}_{\boldsymbol{F}}}\right) \boldsymbol{N}=\mathbf{0} .
$$

Additionally, the directional derivative of the functional (133) with respect to $\left\{\boldsymbol{\Sigma}_{\boldsymbol{F}}^{*}, \Sigma_{J}^{*}\right\}$ results in

$$
\Sigma_{\boldsymbol{F}}^{*}=\boldsymbol{\Sigma}_{\boldsymbol{F}}+\boldsymbol{\lambda} \otimes \boldsymbol{N} ; \quad \Sigma_{J}^{*}=\Sigma_{J}+\boldsymbol{\lambda} \cdot\left(\boldsymbol{H}_{\boldsymbol{\Sigma}_{\boldsymbol{F}}} \boldsymbol{N}\right) .
$$

Substitution of (135) into (134) yields

$$
\boldsymbol{\lambda}=\left[\boldsymbol{I}+\boldsymbol{H}_{\boldsymbol{\Sigma}_{\boldsymbol{F}}} \boldsymbol{N} \otimes \boldsymbol{H}_{\boldsymbol{\Sigma}_{\boldsymbol{F}}} \boldsymbol{N}\right]^{-1}\left(\boldsymbol{t}_{B}-\boldsymbol{P} \boldsymbol{N}\right) .
$$

Finally, combination of expressions (135) and (136) enable the corrected conjugate stresses $\Sigma_{\boldsymbol{F}}^{*}$ and $\Sigma_{J}^{*}$ to be easily obtained. 


\section{Numerical examples}

In this section, a series of numerical examples are presented in order to assess the robustness, effectiveness and applicability of the computational framework described above.

Firstly, the computational framework based on the use of conjugate entropy variables will be briefly analysed and compared against the framework based on the use of conservation variables. For simplicity, two dimensional $\boldsymbol{p}$ - $\boldsymbol{F}$ and $\boldsymbol{p}-\boldsymbol{F}-J$ mixed Petrov-Galerkin Finite Element Method (PG-FEM) formulations will be compared against the entropy $\boldsymbol{v}-\boldsymbol{\Sigma}_{\boldsymbol{F}^{-}} \Sigma_{J}$ mixed PG-FEM formulation. The main objective is to demonstrate that similar results are obtained with either of the approaches, as already reported in [102] for the case compressible Euler flows. The numerical results presented correspond to the use of bilinear interpolation of all the variable fields in a structured quadrilateral mesh. In addition and, for completeness, results will also be presented for a Total Variation Diminishing (TVD) cell-centred Upwind Finite Volume Method (FVM) [50].

Secondly, the framework based on the use of conservation variables will be further explored. With that in mind and, for benchmarking purposes, the new $\boldsymbol{p}-\boldsymbol{F}-\boldsymbol{H}-J$ mixed PG-FEM formulation is tested for a series of three dimensional problems previously analysed by the authors with alternative explicit mixed methodologies [1, 8, 50, 52], namely, a $\boldsymbol{p}$ - $\boldsymbol{F}$ mixed PG-FEM formulation [8], a $\boldsymbol{p}$ - $\boldsymbol{F}$ - $J$ mixed PG-FEM formulation [1] and a $\boldsymbol{p}$-F mixed vertex-centred Jameson-Schmidt-Turkel (JST) formulation [52].

Thirdly, comparisons are also carried out against a new implicit mixed formulation recently proposed by the authors [46] in the context of polyconvex elasticity. A seven field $\left\{\boldsymbol{x}, \boldsymbol{F}, \boldsymbol{H}, J, \boldsymbol{\Sigma}_{\boldsymbol{F}}, \boldsymbol{\Sigma}_{\boldsymbol{H}}, \Sigma_{J}\right\}$ mixed formulation is proposed using a multi-field $\mathrm{Hu}$-Washizu type variational principle. The numerical results presented correspond to the following selection of functional spaces for a tetrahedral mesh: continuous quadratic interpolation of the displacement field (geometry) $\boldsymbol{x}$, piecewise linear interpolation of the strain and stress fields $\boldsymbol{F}, \boldsymbol{H}, \boldsymbol{\Sigma}_{\boldsymbol{F}}$ and $\boldsymbol{\Sigma}_{\boldsymbol{H}}$ and piecewise constant interpolation of the Jacobian $J$ and its associated stress conjugate $\Sigma_{J}$. This selection of functional spaces ensures the satisfaction of the Ladyzenskaya-Babuska-Brezzi (LBB) constraint $[15,104]$ and hence, removes the need to employ numerical stabilisation. From the time discretisation standpoint, a generalised- $\alpha$ method is employed [18] with a built-in numerical damping coefficient $\rho_{\infty}$ in order to dissipate high frequency oscillations. 
The first example (see section 6.1) is included to demonstrate that the use of a conservation-based formulation yields practically identical results to those obtained with a conjugate entropy-based formulation, as already reported in [102] in the context of CFD problems. Focussing on the stabilised three dimensional framework based on the use of conservation variables, it is important to show that the explicit $\boldsymbol{p}-\boldsymbol{F}-\boldsymbol{H}$ - $J$ linear tetrahedral formulation achieves second order of convergence for velocities, strains and stresses (see section 6.2). Perhaps more importantly, the use of the proposed formulation alleviates both locking difficulties (see section 6.3 and section 6.4) and the appearance of spurious hydrostatic oscillations (see section 6.5). Moreover, a thorough comparison of results is carried out against other schemes published by the authors $[1,8,50,52]$. The preservation of the angular momentum of the system is finally assessed in section 6.6.

\subsection{D tensile plate}

A square plate of side length $L=1 \mathrm{~m}$, clamped on its bottom side and traction-free on the rest of the boundaries, was presented in [50]. The plate is made of a Neo-Hookean material defined by Young's modulus $E=21$ GPa, material density $\rho_{0}=7 \mathrm{Mg} / \mathrm{m}^{3}$ and Poisson's ratio $\nu=0.3$. The plate is pulled rapidly with an initial constant velocity field of $\left.\boldsymbol{v}\right|_{t=0}=\left(0, v_{\text {pull }}\right)^{T}$ where $v_{\text {pull }}=500 \mathrm{~m} / \mathrm{s}$.

This problem is first solved using a conjugate entropy $\boldsymbol{v}-\boldsymbol{\Sigma}_{\boldsymbol{F}^{-}} \Sigma_{J}$ formulation. Instead of adopting a user-defined diagonal stabilisation matrix $\boldsymbol{\tau}$, as typically employed in conservation-based formulation (95), a non-diagonal stabilisation matrix $\tilde{\boldsymbol{\tau}}$ is computed following the procedure described in section 5.4.2. For comparison purposes we also solve the problem with a TVD upwind $\boldsymbol{p}$ - $\boldsymbol{F}$ cell centred FVM [50], a $\boldsymbol{p}$-F PG-FEM [8] and a $\boldsymbol{p}$-F-J PG-FEM [1].

Comparison of the deformed shapes and pressure contours obtained by using all of these numerical techniques is shown in Figure 2. The solution predicted by the symmetric $\boldsymbol{v}-\boldsymbol{\Sigma}_{\boldsymbol{F}^{-}} \Sigma_{J}$ conjugate formulation is found to be in perfect agreement with those obtained with the other explicit method-

ologies, showing no appreciable differences. In addition, by making use of the conjugate entropy formulation, a series of pressure contour snapshots are displayed in Figure 3, showing a smooth pressure pattern without any non-physical pressure oscillations. 

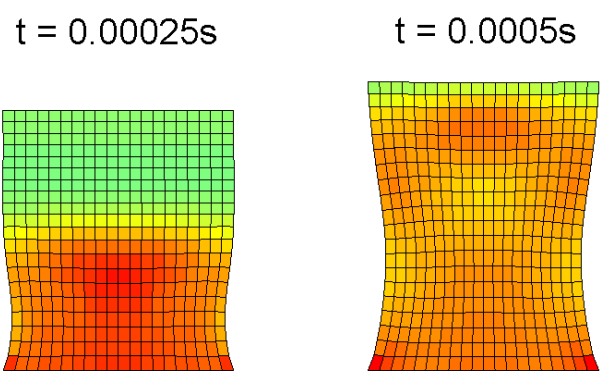

$$
t=0.00075 s
$$

$t=0.001 \mathrm{~s}$

(a) TVD Upwind $\boldsymbol{p}$ - $\boldsymbol{F}$ cell centred FVM [50]
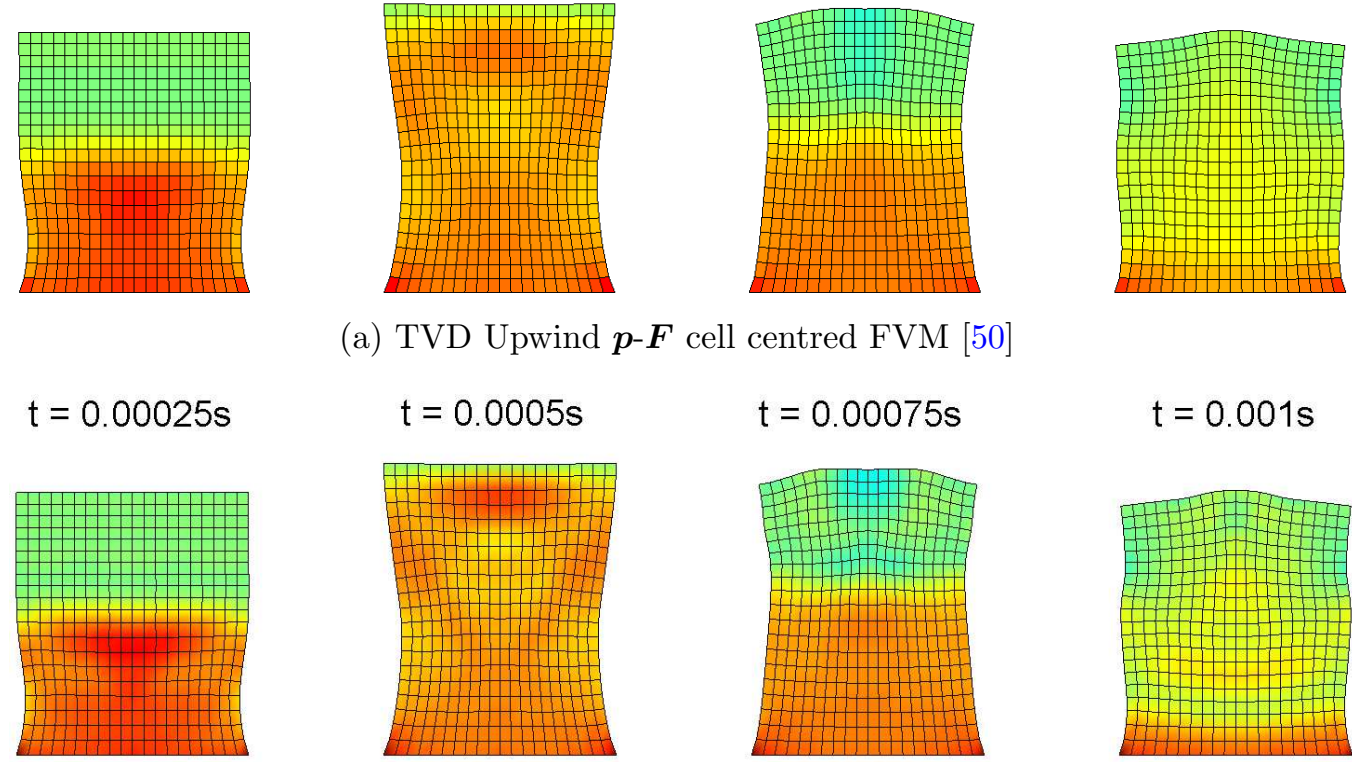

$t=0.001 s$

(b) Stabilised $\boldsymbol{p}$-F PG-FEM [8] $\left(\tau_{\boldsymbol{F}}=\Delta t, \zeta_{\boldsymbol{F}}=0.1, \tau_{\boldsymbol{p}}=0\right)$
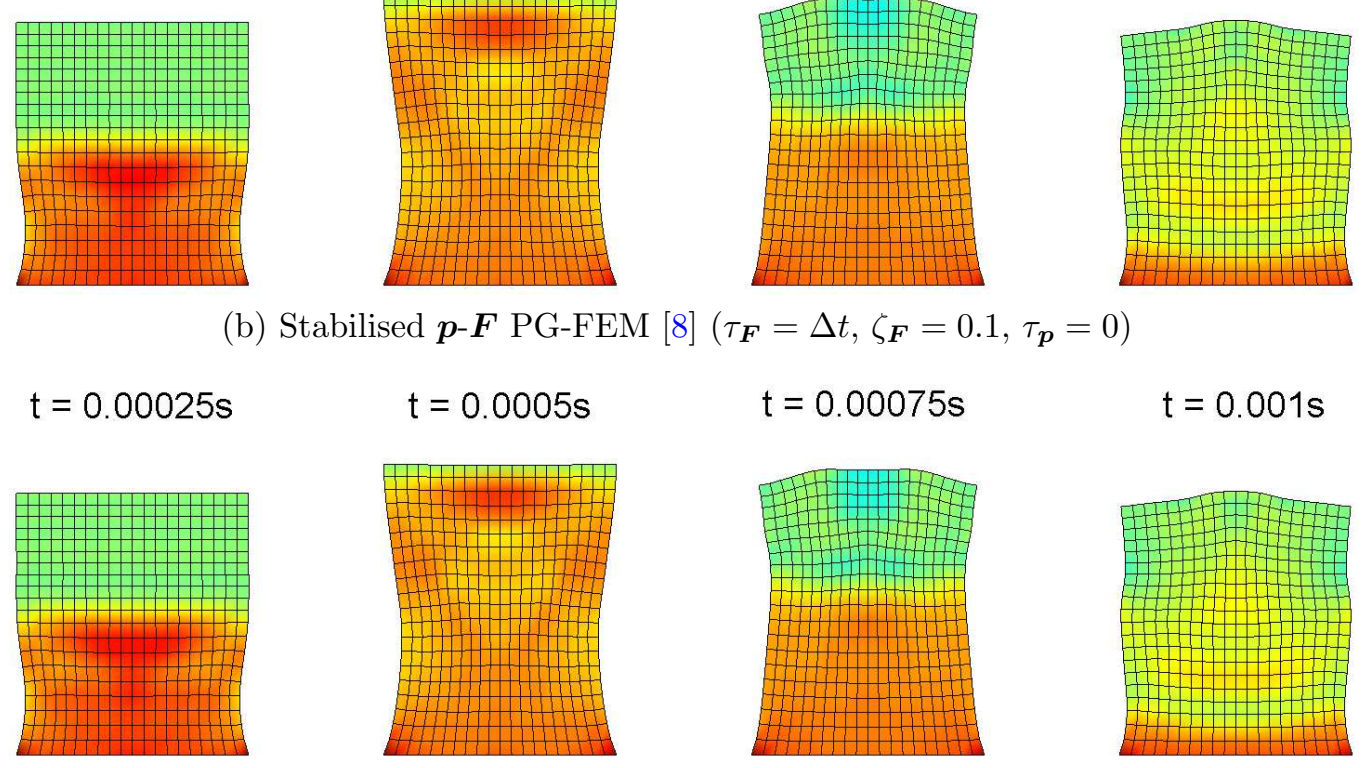

(c) Stabilised $\boldsymbol{p}$ - F-J PG-FEM [1] $\left(\tau_{\boldsymbol{F}}=\Delta t, \tau_{\boldsymbol{p}}=0.2 \Delta t, \zeta_{J}=0.5 \frac{\mu}{\kappa}, \zeta_{\boldsymbol{F}}=\tau_{J}=0\right)$

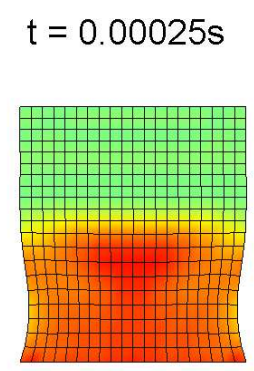

$t=0.0005 s$

$t=0.00075 s$

$\mathrm{t}=0.001 \mathrm{~s}$

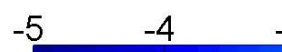

\begin{abstract}
$-3$
\end{abstract}
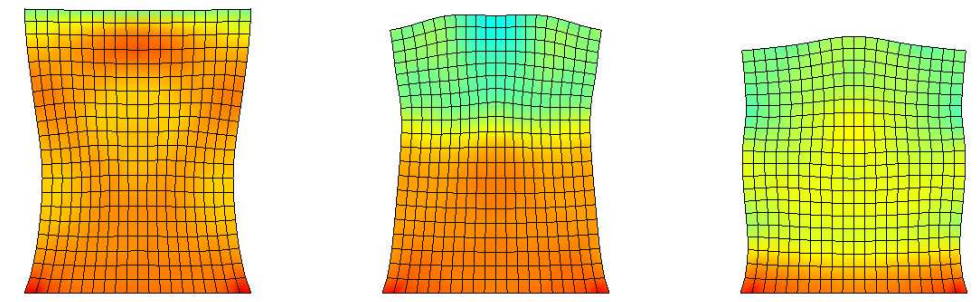

$-2$

12

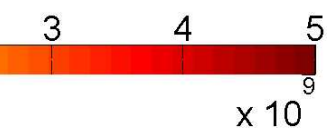

(d) Conjugate entropy $\boldsymbol{v}-\boldsymbol{\Sigma}_{\boldsymbol{F}}-\Sigma_{J}$ formulation (see section 5.4.2)

Figure 2: Tensile plate: sequence of deformed shapes (pressure contour plot) using: (a) TVD $\boldsymbol{p}$ - $\boldsymbol{F}$ cell centred FVM; (b) Stabilised $\boldsymbol{p}$ - $\boldsymbol{F}$ PG-FEM; (c) Stabilised $\boldsymbol{p}-\boldsymbol{F}-J$ PG-FEM; and (d) flntropy $\boldsymbol{v}-\boldsymbol{\Sigma}_{\boldsymbol{F}^{-}} \Sigma_{J}$ formulation. Initial constant tensile velocity $\left.\boldsymbol{v}\right|_{t=0}=\left(0, v_{\text {pull }}\right)^{T}$ is given, where $v_{\text {pull }}=500 \mathrm{~m} / \mathrm{s}$. A steel plate is used and its Neo-Hookean material properties are Young's modulus $E=21 \mathrm{GPa}$, density $\rho_{0}=7 \mathrm{Mg} / \mathrm{m}^{3}$, Poisson's ratio $\nu=0.3$ and $\alpha_{\mathrm{CFL}} \approx 0.3$. Discretisation of $20 \times 20$ quadrilateral elements. 

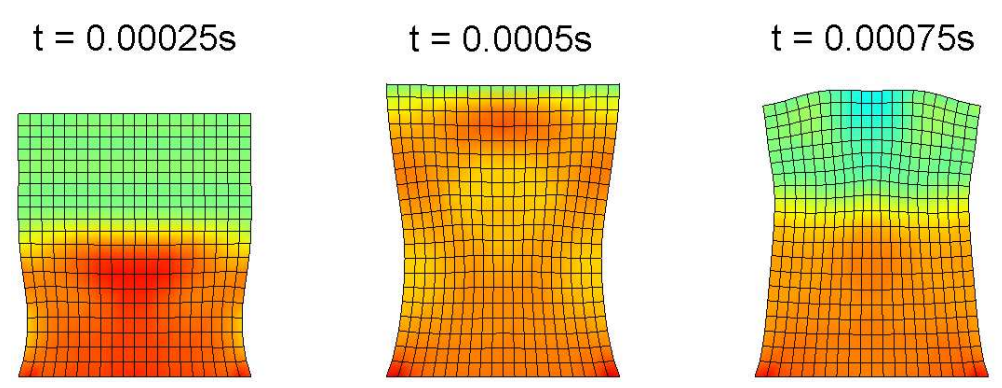

$$
t=0.001 s
$$

$t=0.00125 s$

$$
t=0.0015 s
$$

$$
t=0.002 s
$$

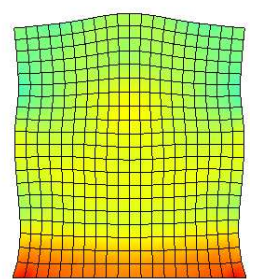

$t=0.0025 s$
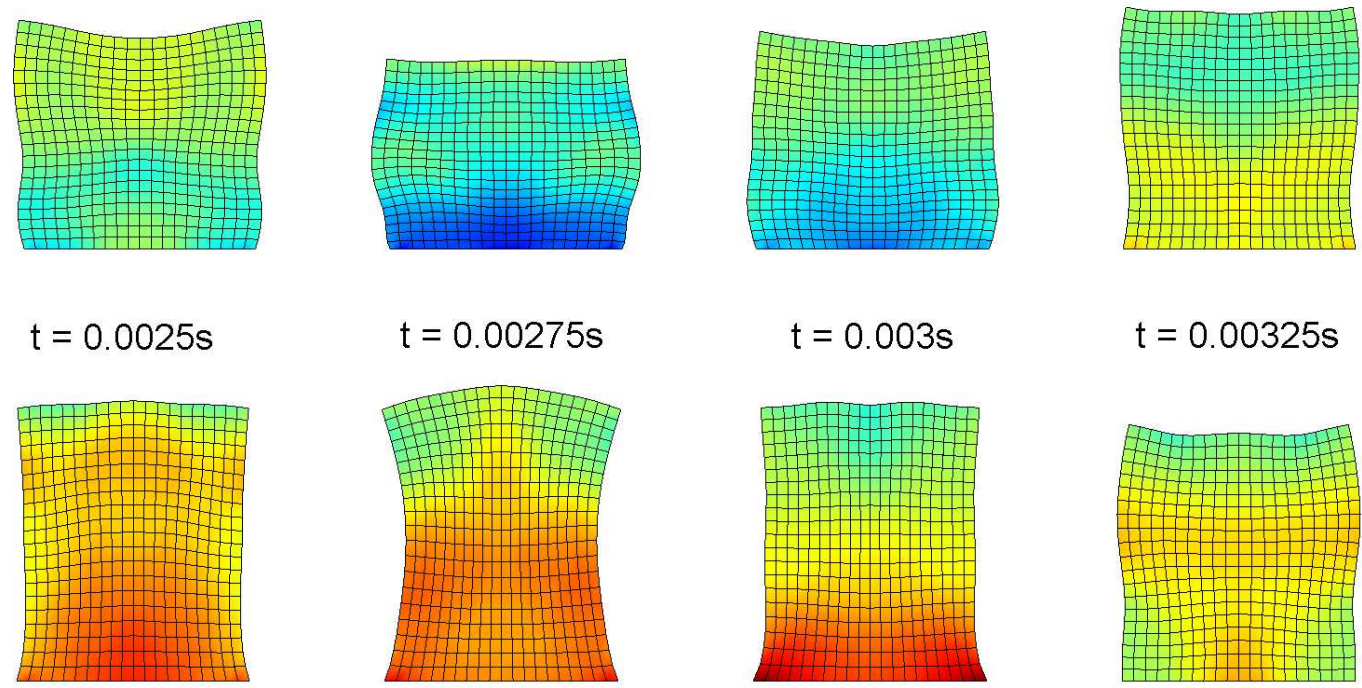

$$
t=0.003 s
$$$$
t=0.00325 s
$$
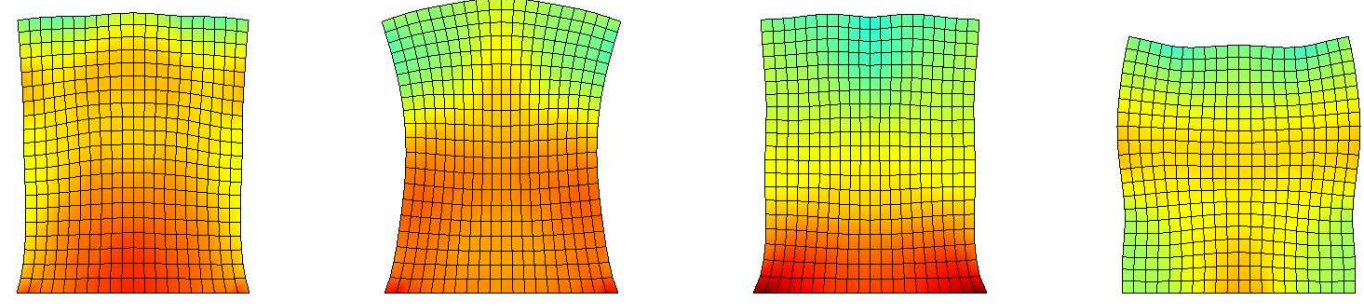

$$
t=0.0035 s
$$

$$
t=0.00425 s
$$

$$
t=0.0045 s
$$

$$
\mathrm{t}=0.005 \mathrm{~s}
$$
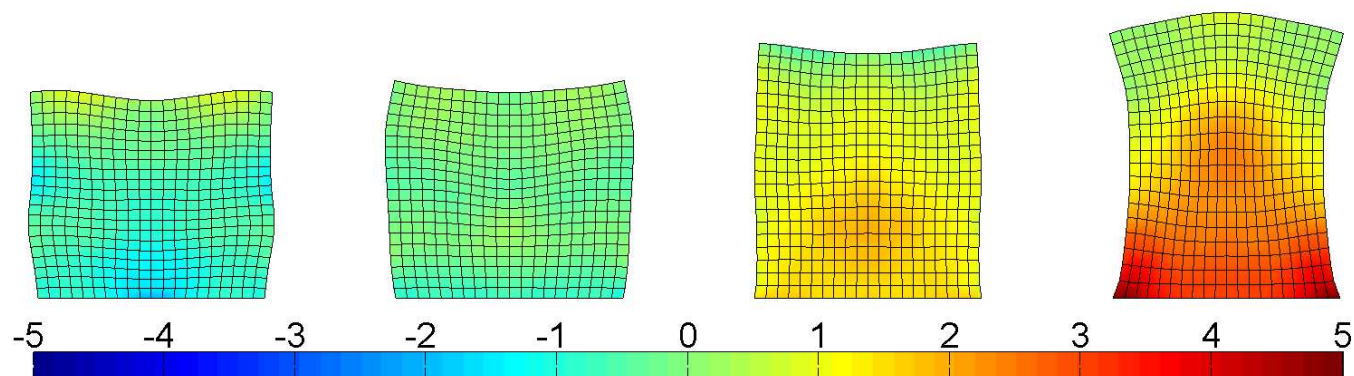

3

4

$\times 10$

Figure 3: Tensile plate: sequence of deformed shapes (pressure contour plot) using the conjugate entropy $\boldsymbol{v}-\boldsymbol{\Sigma}_{\boldsymbol{F}^{-}} \Sigma_{J}$ formulation. Initial constant tensile velocity $\left.\boldsymbol{v}\right|_{t=0}=\left(0, v_{\text {pull }}\right)^{T}$ is given, where $v_{\text {pull }}=500 \mathrm{~m} / \mathrm{s}$. A steel plate is used and its Neo-Hookean material properties are Young's modulus $E=$ $21 \mathrm{GPa}$, density $\rho_{0}=7 \mathrm{Mg} / \mathrm{m}^{3}$, Poisson's ratio $\nu=0.3$ and $\alpha_{\mathrm{CFL}} \approx 0.3$. Discretisation of $20 \times 20$ quadrilateral elements. 


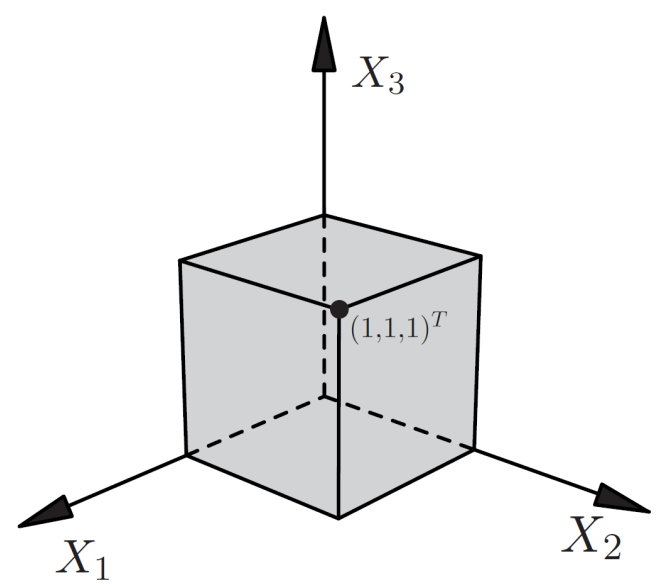

Figure 4: Unit cube configuration

\subsection{D low dispersion cube}

This example has already been presented in previous references $[1,52]$. A unit cube $(1 \mathrm{~m} \times 1 \mathrm{~m} \times 1 \mathrm{~m})$ is defined with symmetric boundary conditions (i.e. roller supports) applied on the faces $X_{1}=X_{2}=X_{3}=0$ and skewsymmetric boundary conditions on the rest of the faces $X_{1}=X_{2}=X_{3}=1$ $\mathrm{m}$ (see Figure 4). The primary interest is to assess the convergence behaviour of the algorithm in three dimensions. For small deformations, the analytical displacement field (and hence, velocity and first Piola-Kirchhoff stress) can be described by the closed-form expression

$$
\boldsymbol{u}(\boldsymbol{X}, t)=U_{0} \cos \left(\frac{\sqrt{3}}{2} c_{d} \pi t\right)\left[\begin{array}{c}
A \sin \left(\frac{\pi X_{1}}{2}\right) \cos \left(\frac{\pi X_{2}}{2}\right) \cos \left(\frac{\pi X_{3}}{2}\right) \\
B \cos \left(\frac{\pi X_{1}}{2}\right) \sin \left(\frac{\pi X_{2}}{2}\right) \cos \left(\frac{\pi X_{3}}{2}\right) \\
C \cos \left(\frac{\pi X_{1}}{2}\right) \cos \left(\frac{\pi X_{2}}{2}\right) \sin \left(\frac{\pi X_{3}}{2}\right)
\end{array}\right] ; c_{d}=\sqrt{\frac{\mu}{\rho_{0}}} .
$$

Parameters $\{A, B, C\}$ are arbitrary constants such that $A+B+C=0$, ensuring no contribution from volumetric deformation. For values of $U_{0}$ below $0.001 \mathrm{~m}$, the solution can be considered to be linear and the closed-form solution holds. The cube is initially loaded (without imposing any initial velocity field) with a known deformation gradient field $\boldsymbol{F}$ (by computing $\boldsymbol{F}=\boldsymbol{I}+\boldsymbol{\nabla}_{0} \boldsymbol{u}$ with $\boldsymbol{u}$ obtained from (137)). The initial area map $\boldsymbol{H}=$ $(\operatorname{det} \boldsymbol{F}) \boldsymbol{F}^{-T}$ and the initial Jacobian $J=\operatorname{det} \boldsymbol{F}$ are similarly obtained. 
In this particular case, a compressible Neo-Hookean model $\left(\alpha=\frac{\mu}{2}\right.$ and $\beta=0$ ) is chosen with Young's modulus $E=0.017 \mathrm{GPa}$, density $\rho_{0}=1.1$ $\mathrm{Mg} / \mathrm{m}^{3}$ and Poisson's ratio $\nu=0.3$. We set the solution parameters as $A=$ $B=1, C=-2$ and $U_{0}=5 \times 10^{-4} \mathrm{~m}$. Figure 5 shows a series of snapshots for deformed states of the cube illustrating shear stresses contour plots (which coincide with the results reported in references $[1,52])$. Global convergence error analysis (i.e. $L^{1}$ and $L^{2}$ norm convergence) for the set of conservation $\boldsymbol{p}-\boldsymbol{F}-\boldsymbol{H}-J$ variables and the Piola stress tensor $\boldsymbol{P}$ (e.g. $\|\boldsymbol{p}\|_{L^{1,2}},\|\boldsymbol{F}\|_{L^{1,2}}$, $\|\boldsymbol{H}\|_{L^{1,2}},\|J\|_{L^{1,2}}$ and $\left.\|\boldsymbol{P}\|_{L^{1,2}}\right)$ on a sequence of grids at time $t=2 \times 10^{-3} \mathrm{~S}$ are examined. In this case, the stabilised $\boldsymbol{p}-\boldsymbol{F}-\boldsymbol{H}-J$ mixed PG-FEM approach is employed. As expected, Figure 6 shows asymptotic quadratic convergence for all problem variables (and derived variables) when linear tetrahedral finite elements are used.

\section{3. $3 D$ beam bending}

In this section, a three dimensional thick column [1, 10, 52] clamped on its bottom face $\left(X_{3}=0\right)$ is presented in Figure 7 . An initial linear variation in the velocity field $\left.\boldsymbol{v}\right|_{t=0}=\left(V_{0} X_{3} / L, 0,0\right)^{T}$ is given (where $V_{0}=10 \mathrm{~m} / \mathrm{s}$ ) and the column is left oscillating in time, leading to a large oscillatory deformation. The main objective of this problem is to illustrate the performance of the new $\boldsymbol{p}$ - $\boldsymbol{F}-\boldsymbol{H}$ - $J$ mixed PG-FEM formulation in a compressible bending dominated scenario.

A Mooney-Rivlin material $\left(\alpha=\beta=\frac{\mu}{4}\right)$ is employed such that Young's modulus $E=0.017 \mathrm{GPa}$, density $\rho_{0}=1.1 \mathrm{Mg} / \mathrm{m}^{3}$ and Poisson's ratio $\nu=$ 0.3. For comparison purposes, the problem is also studied with the seven field $\left\{\boldsymbol{x}, \boldsymbol{F}, \boldsymbol{H}, J, \boldsymbol{\Sigma}_{\boldsymbol{F}}, \boldsymbol{\Sigma}_{\boldsymbol{H}}, \Sigma_{J}\right\}$ implicit $\mathrm{Hu}$-Washizu type variational formulation [46] described above as well as with a set of explicit numerical strategies (e.g. vertex-centred JST finite volume mixed $\boldsymbol{p}-\boldsymbol{F}$ formulation [52] and the stabilised $\boldsymbol{p}-\boldsymbol{F}$ [8] and $\boldsymbol{p}-\boldsymbol{F}-J$ [1] PG formulations).

Figure 8a shows the (bending) locking-free deformed shapes of a thick column at four different time steps using the explicit stabilised $\boldsymbol{p}-\boldsymbol{F}-\boldsymbol{H}-J$ PG-FEM methodology. Comparison of Figure 8a (p-F-H-J PG-FEM) with Figure 8b,c (alternative $\boldsymbol{p}$ - $\boldsymbol{F}$ and $\boldsymbol{p}$ - $\boldsymbol{F}$ - $J$ PG-FEM) and Figure 9 (vertexcentred JST $\boldsymbol{p}$ - $\boldsymbol{F}$ FVM and Hu-Washizu type mixed formulation), clearly demonstrates that the proposed PG formulation can be used without bending difficulty and, more importantly, shows excellent agreement with the previously published methodologies. In all cases, the pressure contours are extremely smooth without any non-physical oscillations. 


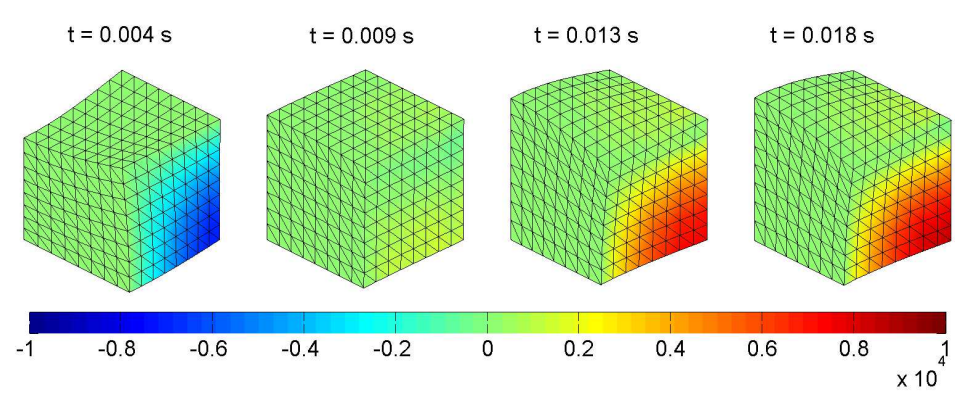

(a) Shear stress $P_{12}$

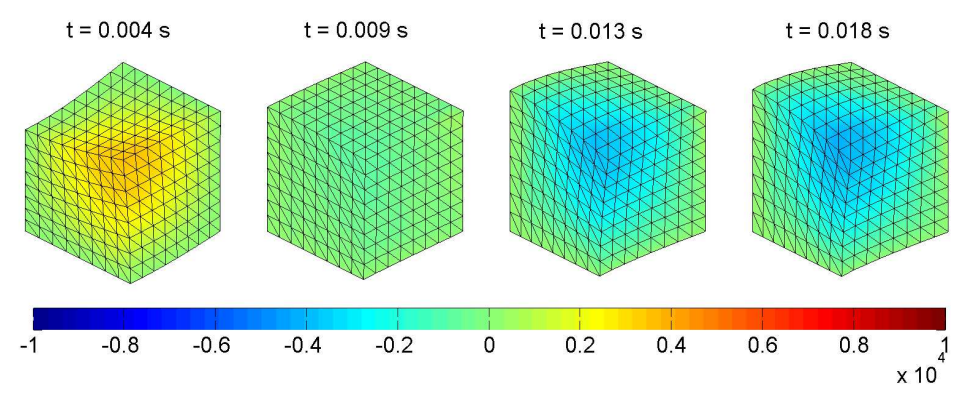

(b) Shear stress $P_{13}$

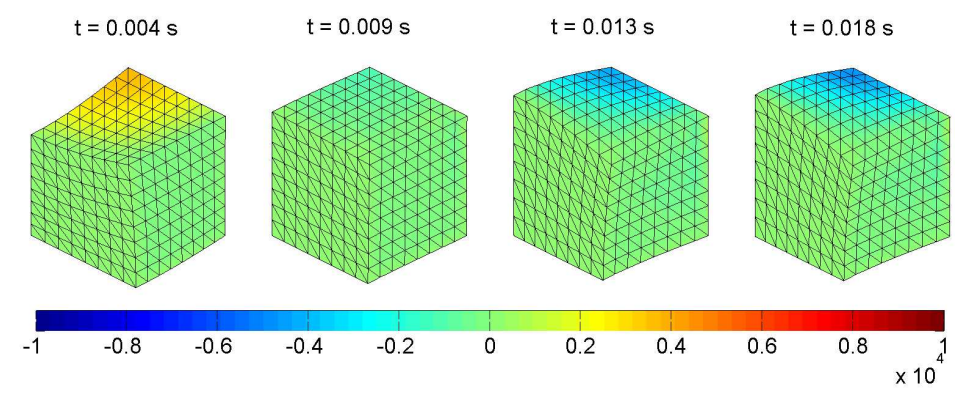

(c) Shear stress $P_{23}$

Figure 5: Low dispersion cube: sequence of deformed shapes for (a) $P_{12}$ shear stress; (b) $P_{13}$ shear stress; and (c) $P_{23}$ shear stress. Results obtained with $U_{0}=5 \times 10^{-4} \mathrm{~m}, A=B=1$ and $C=-2$. Compressible Neo-Hookean model is used and material properties are such that Young's modulus $E=0.017$ GPa, density $\rho_{0}=1.1 \mathrm{Mg} / \mathrm{m}^{3}$, Poisson's ratio $\nu=0.3$ and $\alpha_{C F L}=0.3$. Stabilising parameters: $\tau_{\boldsymbol{F}}=\tau_{\boldsymbol{H}}=\Delta t, \tau_{\boldsymbol{p}}=0.2 \Delta t, \zeta_{\boldsymbol{F}}=\zeta_{\boldsymbol{H}}=\tau_{J}=0$ and $\zeta_{J}=0.5 \frac{\mu}{\kappa}$. Discretisation of $8 \times 8 \times 8 \times 6$ linear tetrahedral elements. Lumped mass contribution. 

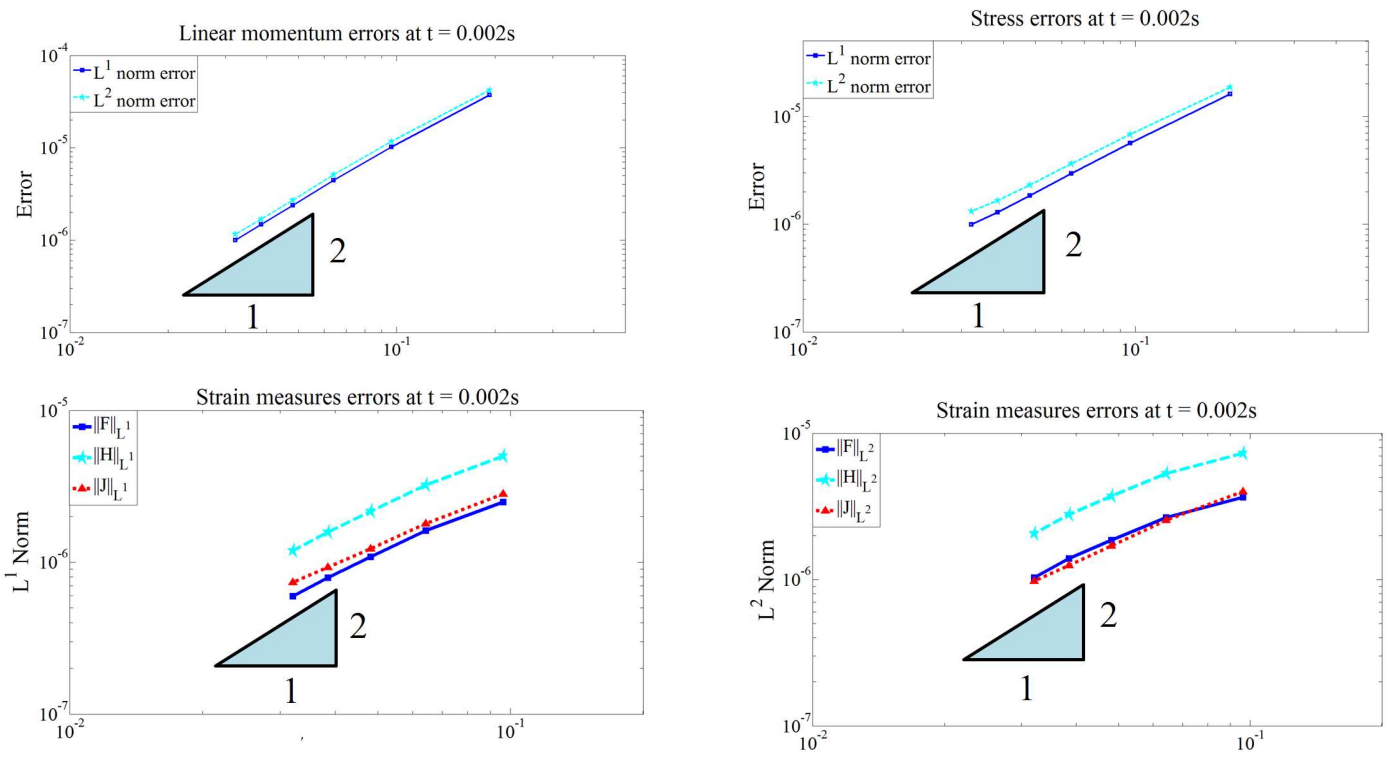

Figure 6: Low dispersion cube: results obtained with $U_{0}=5 \times 10^{-4} \mathrm{~m}$, $A=B=1$ and $C=-2$, where the analytical solutions for velocity, strains and stresses are available at time $t=2 \times 10^{-3} \mathrm{~s}$. First row shows the $\mathrm{L}^{1}$ and $L^{2}$ norm convergences for linear momentum $\|\boldsymbol{p}\|_{L^{1,2}}$ and stresses $\|\boldsymbol{P}\|_{L^{1,2}}$. Second row shows the norm convergence analysis for all strain measures (i.e. $\|\boldsymbol{F}\|_{L^{1,2}},\|\boldsymbol{H}\|_{L^{1,2}}$ and $\left.\|J\|_{L^{1,2}}\right)$. A compressible Neo-Hookean elastic model $\left(\alpha=\frac{\mu}{2}\right.$ and $\left.\beta=0\right)$ is used and material properties are such that Young's modulus $E=0.017 \mathrm{GPa}$, density $\rho_{0}=1.1 \mathrm{Mg} / \mathrm{m}^{3}$, Poisson's ratio $\nu=0.3$ and $\alpha_{C F L}=0.3$. Stabilising parameters: $\tau_{\boldsymbol{F}}=\tau_{\boldsymbol{H}}=\Delta t, \tau_{\boldsymbol{p}}=0.2 \Delta t$, $\zeta_{J}=0.5 \frac{\mu}{\kappa}$ and $\zeta_{\boldsymbol{F}}=\zeta_{\boldsymbol{H}}=\tau_{J}=0$. Lumped mass contribution. 


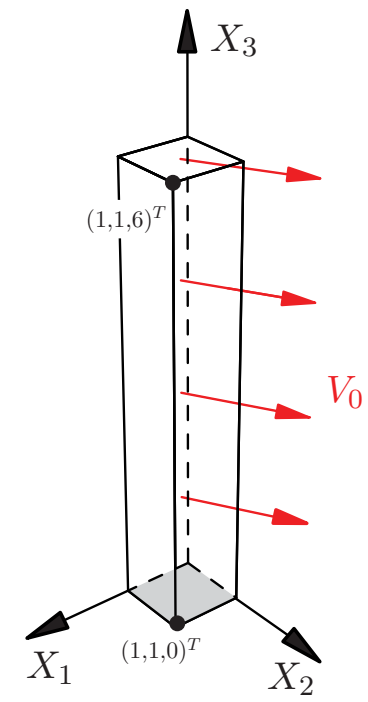

Figure 7: Bending column configuration 


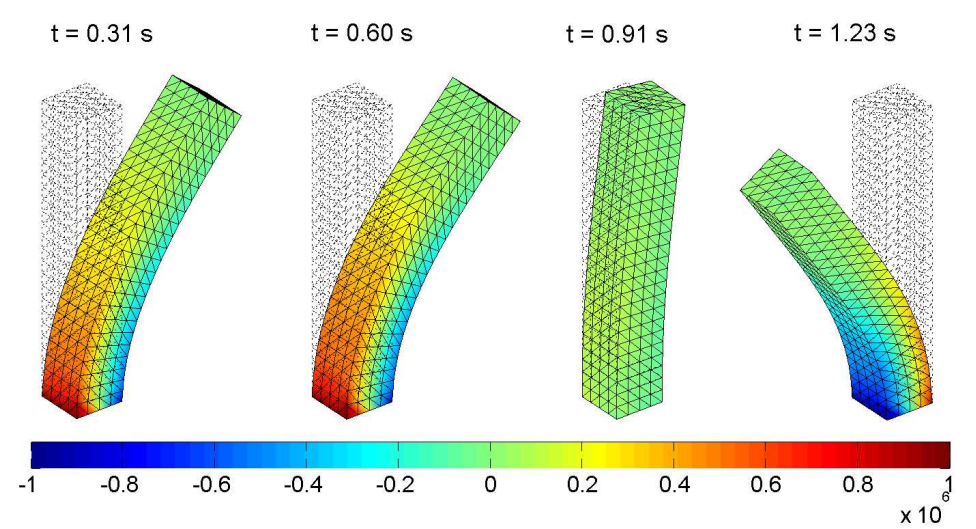

(a) Stabilised $\boldsymbol{p}-\boldsymbol{F}-\boldsymbol{H}-J$ PG-FEM $\left(\tau_{\boldsymbol{F}}=\tau_{\boldsymbol{H}}=\Delta t, \tau_{\boldsymbol{p}}=0.2 \Delta t\right.$, $\left.\zeta_{J}=0.5 \frac{\mu}{\kappa}, \zeta_{\boldsymbol{F}}=\zeta_{\boldsymbol{H}}=\tau_{J}=0\right)$

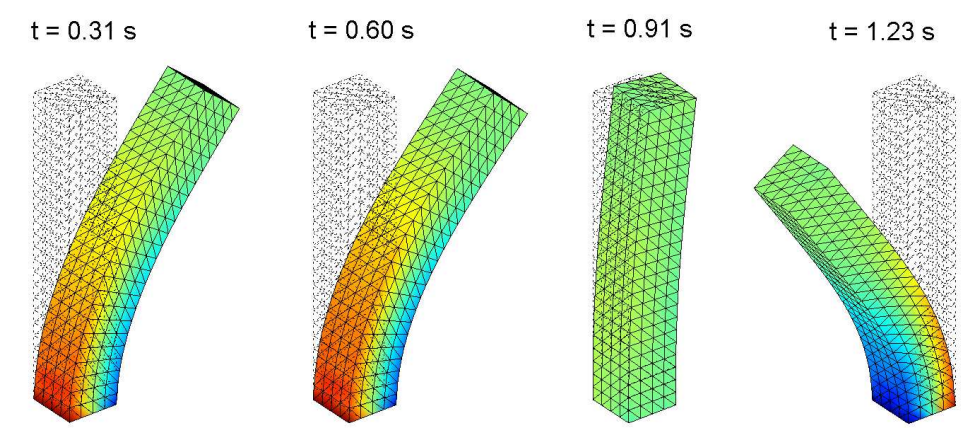

(b) Stabilised $\boldsymbol{p}$ - $\boldsymbol{F}$ PG-FEM $\left(\tau_{\boldsymbol{F}}=\Delta t, \zeta_{\boldsymbol{F}}=0.1, \tau_{\boldsymbol{p}}=0\right)$

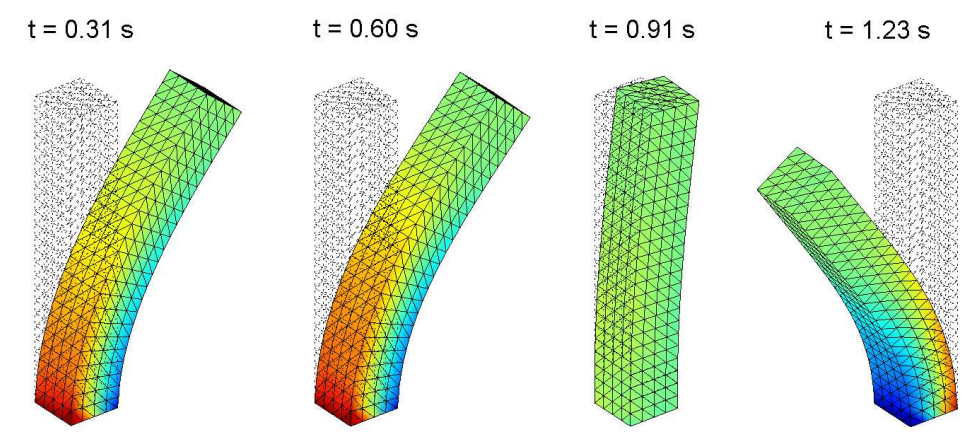

(c) Stabilised $\boldsymbol{p}$ - $\boldsymbol{F}$ - $J$ PG-FEM $\left(\tau_{\boldsymbol{F}}=\Delta t, \tau_{\boldsymbol{p}}=0.2 \Delta t, \zeta_{J}=0.5 \frac{\mu}{\kappa}\right.$, $\left.\zeta_{\boldsymbol{F}}=\tau_{J}=0\right)$

Figure 8: Bending column: sequence of deformed shapes (pressure contour plot) using: (a) Stabilised $\boldsymbol{p}-\boldsymbol{F}-\boldsymbol{H}$-J PG-FEM; (b) Stabilised $\boldsymbol{p}-\boldsymbol{F}$ PG-FEM; and (c) Stabilised $\boldsymbol{p}$ - $\boldsymbol{F}-J$ PG-FEM. Results obtained with a linear variation in velocity field $\left.\boldsymbol{v}\right|_{t=0}=\left(V_{0} X_{3} / L, 0,0\right)^{T}$ (where $V_{0}=10 \mathrm{~m} / \mathrm{s}$ ). A compressible Mooney-Rivlin material $\left(\alpha=\beta=\frac{\mu}{4}\right)$ is used such that Young's modulus $E=0.017 \mathrm{GPa}$, density $\rho_{0}=1.1 \mathrm{Mg} / \mathrm{m}^{3}$ and Poisson's ratio $\nu=0.3$. Discretisation of $4 \times 4 \times 24 \times 6$ linear tetrahedral elements. Lumped mass contribution. 


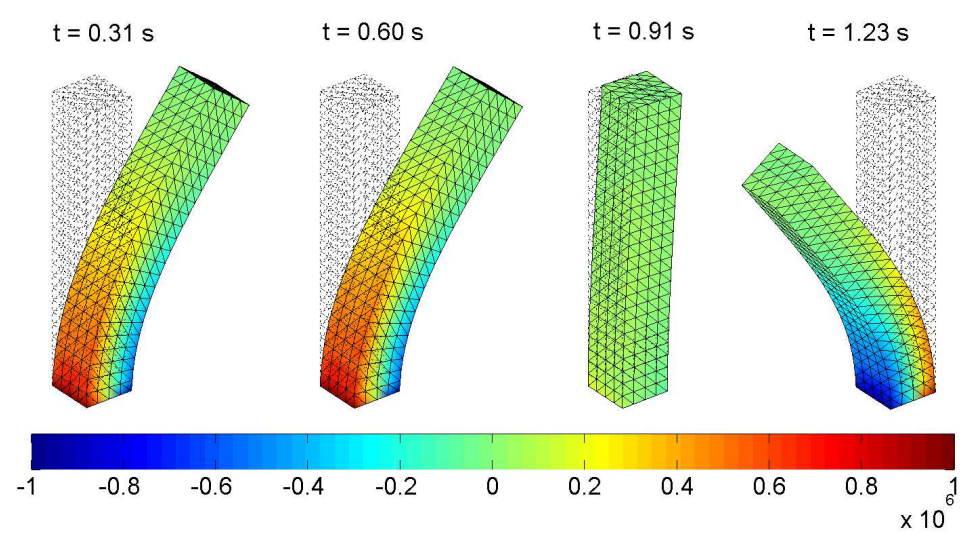

(a) JST $\boldsymbol{p}-\boldsymbol{F}$ vertex centred FVM

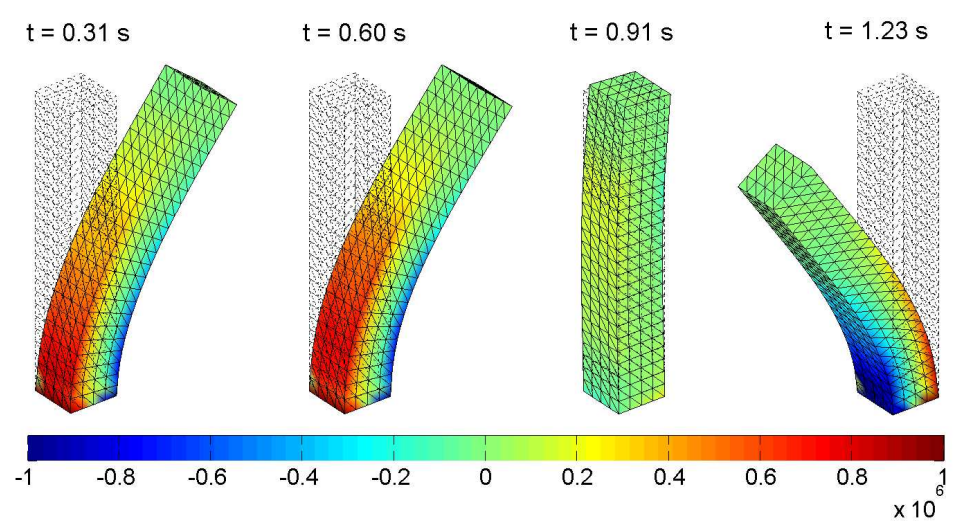

(b) Hu-Washizu type variational formulation

Figure 9: Bending column: sequence of deformed shapes (pressure contour plot) using: (a) JST vertex-centred mixed $\boldsymbol{p}-\boldsymbol{F}$ formulation $\left(\kappa^{(4)}=\frac{1}{128}\right.$ and $\left.\alpha_{C F L}=0.3\right)$; and (b) Hu-Washizu type variational formulation $\left(\rho_{\infty}=1\right.$ and $\Delta t=0.002 \mathrm{~s})$. Results obtained with a linear variation in velocity field $\left.\boldsymbol{v}\right|_{t=0}=\left(V_{0} X_{3} / L, 0,0\right)^{T}$ (where $V_{0}=10 \mathrm{~m} / \mathrm{s}$ ). A compressible MooneyRivlin material $\left(\alpha=\beta=\frac{\mu}{4}\right)$ is used such that Young's modulus $E=0.017$ GPa, density $\rho_{0}=1.1 \mathrm{Mg} / \mathrm{m}^{3}$ and Poisson's ratio $\nu=0.3$. Discretisation of $4 \times 4 \times 24 \times 6$ tetrahedral elements. 


\subsection{D twisting column}

To assess the robustness of the stabilised $\boldsymbol{p}-\boldsymbol{F}-\boldsymbol{H}$ - $J$ PG mixed formulation in extreme nonlinear deformations, a twisting column clamped on its bottom face $\left(X_{3}=0\right)$ is presented $[1,46]$ (see Figure 10). This problem is particularly challenging as it involves a large number of deformation modes. An initial sinusoidal rotational velocity field relative to the origin is given by

$$
\left.\boldsymbol{v}\right|_{t=0}(\boldsymbol{X})=\boldsymbol{\omega}_{0} \times \boldsymbol{X} ; \quad \boldsymbol{\omega}_{0}=\left(0,0, \Omega \sin \left(\frac{\Pi X_{3}}{2 L}\right)\right)^{T}
$$

where $\Omega=100 \mathrm{rad} / \mathrm{s}$. This problem is first modelled using a compressible Mooney-Rivlin material with parameters $\alpha=\beta=\frac{\mu}{4}$. Material properties are Young's modulus $E=0.017 \mathrm{GPa}$, material density $\rho_{0}=1.1 \mathrm{Mg} / \mathrm{m}^{3}$ and Poisson's ratio $\nu=0.3$. In parallel, for benchmarking purposes, we simulate the exact same problem using other available methodologies, namely the stabilised $\boldsymbol{p}-\boldsymbol{F}$ and $\boldsymbol{p}-\boldsymbol{F}-J$ PG-FEM, Hu-Washizu type mixed formulation and the vertex-centred $\boldsymbol{p}$ - $\boldsymbol{F}$ JST formulation.

It can be observed that the deformation patterns predicted by the family of second order stabilised numerical mixed methodologies (e.g. a stabilised $\boldsymbol{p}$ $\boldsymbol{F}$ PG-FEM [8], $\boldsymbol{p}-\boldsymbol{F}-J$ PG-FEM [1] and $\boldsymbol{p}-\boldsymbol{F}-\boldsymbol{H}-J$ PG-FEM) agree very well (see Figure 11). Crucially, all computational mixed methodologies produce very similar deformation patterns with smooth pressure distribution and absence of locking. In Figure 12a, results are also presented for a JST vertex centred $\boldsymbol{p}$ - $\boldsymbol{F}$ FVM solver [52]. In this case, a finer mesh of $12 \times 12 \times 72 \times 6$ linear tetrahedral elements is required in order to obtain similar results, due to the higher numerical diffusion introduced by the scheme.

Finally, comparison is carried out against a more expensive implicit $\mathrm{Hu}-$ Washizu type variational formulation of $4 \times 4 \times 24 \times 6$ tetrahedral elements (see Figure 12b) with an implicit generalised- $\alpha$ method. As can be observed, despite using an artificial numerical damping coefficient $\rho_{\infty}<1$, some higher pressure modes can still be perceived in the solution.

We can now further examine the problem by scaling up the $\beta$ parameter so that the Mooney-Rivlin modelx is now dominated by the $\boldsymbol{H}$-term $(\alpha=0$ and $\beta=\frac{\mu}{2}$ ). Figure 13 shows the effects of the inclusion of time-integrated $\zeta_{\boldsymbol{H}}$ values in the $\mathrm{PG}$ formulation at a particular time $t=0.31 \mathrm{~s}$, whilst the remaining stabilisation terms are kept constant.

It is interesting to notice that the stabilisation parameter $\zeta_{\boldsymbol{H}}$ becomes increasingly important when simulating a Mooney-Rivlin model entirely dom- 
inated by the $\boldsymbol{H}$ term. The PG formulation incorporating the $\zeta_{\boldsymbol{H}}$-term eliminates the appearance of non-physical mechanisms similar to hourglassing in the solution (see Figure 13a,b). An out-of-plane mode of deformation is observed for a very coarse mesh $(4 \times 4 \times 24 \times 6$ linear tetrahedral elements). However, this mode disappears when the mesh is sufficiently refined (see Figure 13c).

Figure 14 shows the comparison of a sequence of snapshots for the highly nonlinear column at four different time instants using the stabilised $\boldsymbol{p}$ - $\boldsymbol{F}$ $\boldsymbol{H}$ - $J$ PG-FEM and the extended Hu-Washizu type mixed formulation. Both methodologies produce practically similar locking-free solutions. As expected, despite using $\rho_{\infty}<1$, some high frequency pressure modes can still be observed in Figure 14b.

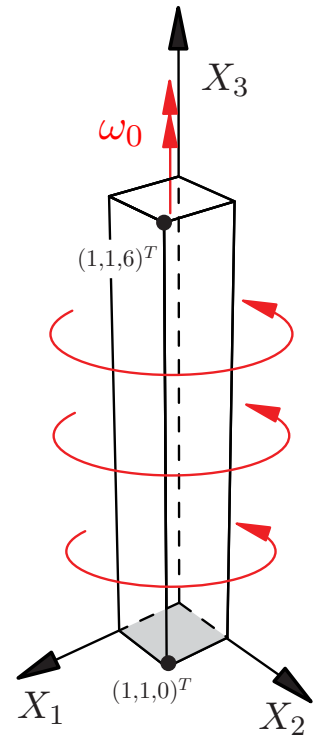

Figure 10: Highly nonlinear twisting column 


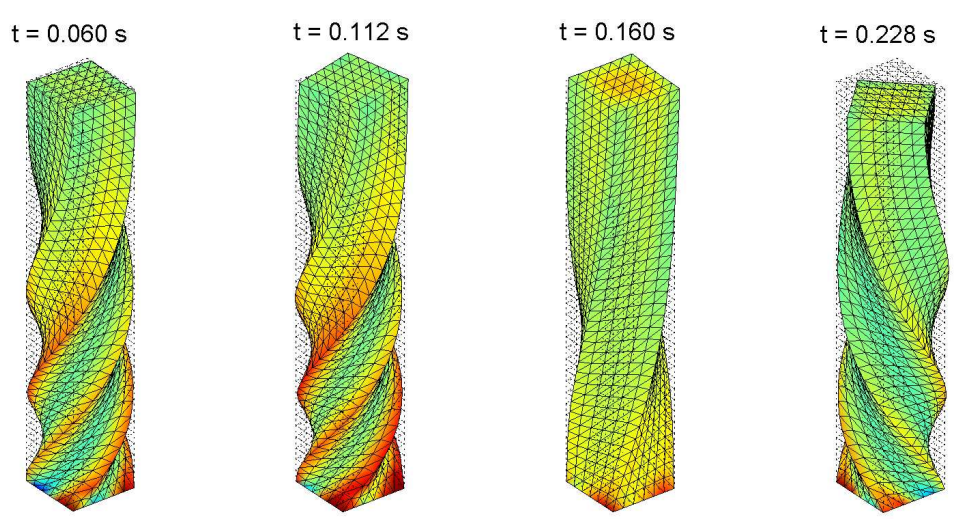

(a) Stabilised $\boldsymbol{p}$ - $\boldsymbol{F}$ PG-FEM $\left(\tau_{\boldsymbol{F}}=\Delta t, \zeta_{\boldsymbol{F}}=0.2, \tau_{\boldsymbol{p}}=0\right)$
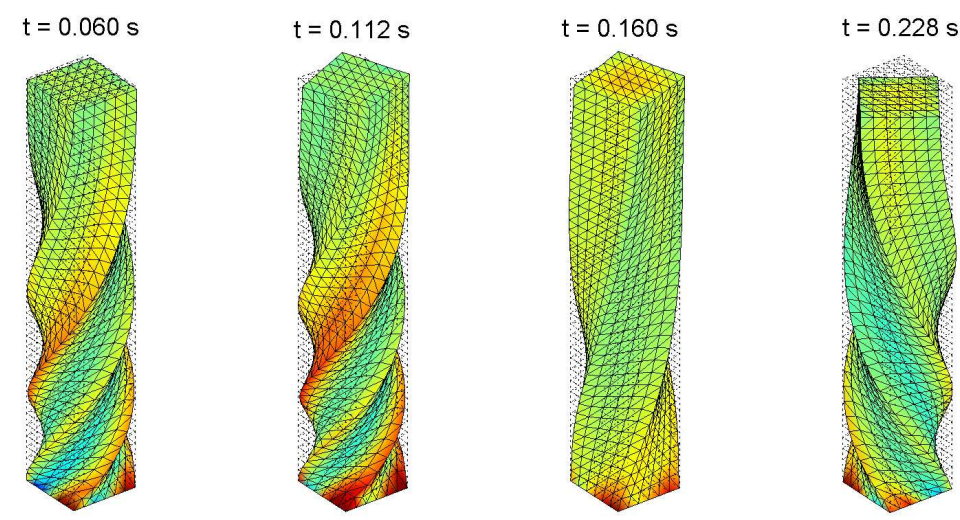

(b) Stabilised $\boldsymbol{p}$-F-J PG-FEM $\left(\tau_{\boldsymbol{F}}=\Delta t, \tau_{\boldsymbol{p}}=0.2 \Delta t, \zeta_{J}=\right.$ $\left.0.5 \frac{\mu}{\kappa}, \zeta_{\boldsymbol{F}}=\tau_{J}=0\right)$

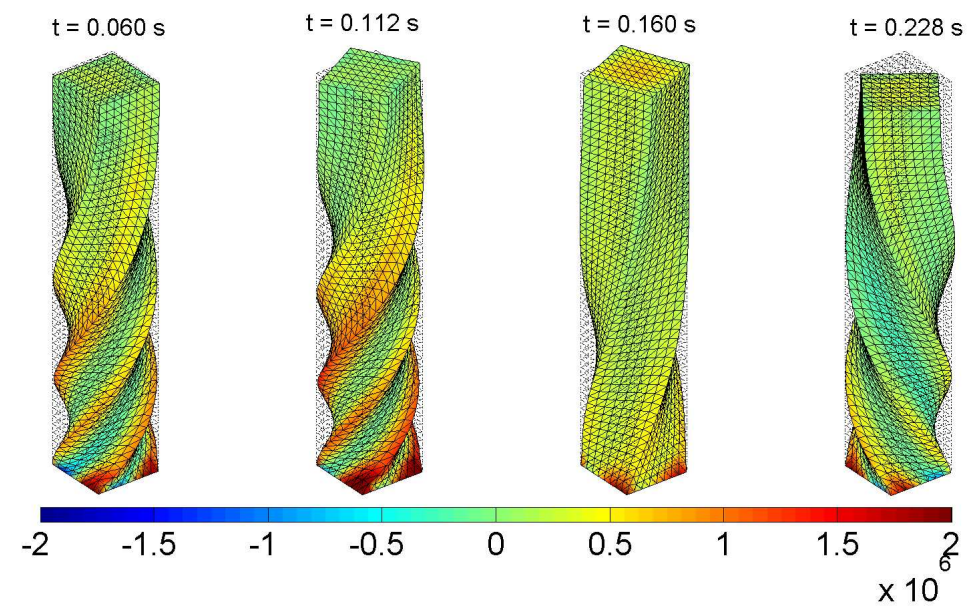

(c) Stabilised $\boldsymbol{p}$ - $\boldsymbol{F}$ - $\boldsymbol{H}-J$ PG-FEM $\left(\tau_{\boldsymbol{F}}=\tau_{\boldsymbol{H}}=\Delta t, \tau_{\boldsymbol{p}}=0.2 \Delta t\right.$, $\left.\zeta_{J}=0.5 \frac{\mu}{\kappa}, \zeta_{\boldsymbol{F}}=\zeta_{\boldsymbol{H}}=\tau_{J}=0\right)$

Figure 11: Twisting column: sequence of deformed shapes (pressure contour plot) using: (a) Stabilised $\boldsymbol{p}$ - $\boldsymbol{F}$ PG-FEM $(6 \times 6 \times 36 \times 6$ linear tetrahedral elements); (b) Stabilised $\boldsymbol{p}$-F-J PG-FEM $(6 \times 6 \times 36 \times 6$ linear tetrahedral elements); and (c) Stabilised $\boldsymbol{p}$ - $\boldsymbol{F}^{2}-\boldsymbol{H}$-J PG-FEM $(8 \times 8 \times 48 \times 6$ linear tetrahedral elements). Results obtained with a initial sinusoidal rotational velocity $\Omega=100 \mathrm{rad} / \mathrm{s}$ (see (138)). A compressible Mooney-Rivlin material $\left(\alpha=\beta=\frac{\mu}{4}\right)$ is used such that Young's modulus $E=0.017 \mathrm{GPa}$, density $\rho_{0}=1.1 \mathrm{Mg} / \mathrm{m}^{3}$, Poisson's ratio $\nu=0.3$ and $\alpha_{C F L}=0.3$. Lumped mass contribution. 

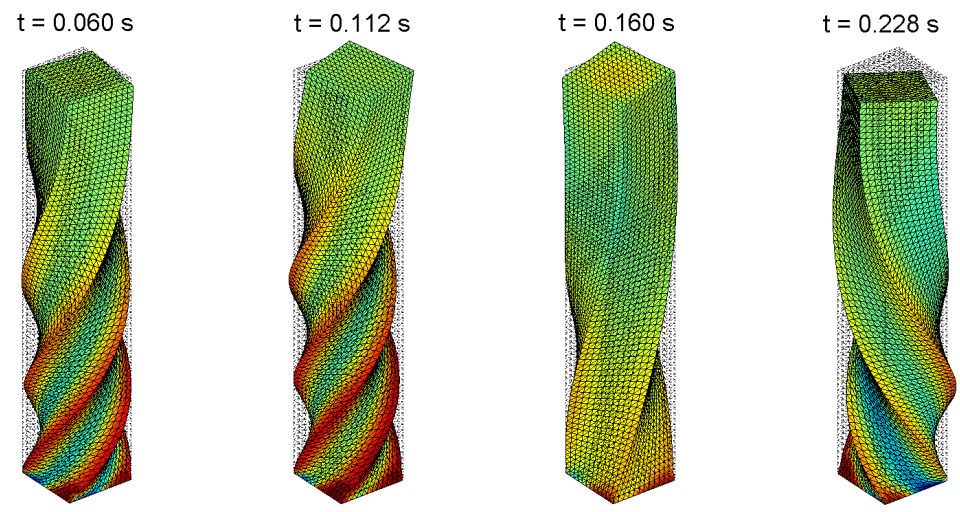

(a) JST vertex centred $\boldsymbol{p}$ - $\boldsymbol{F}$ FVM

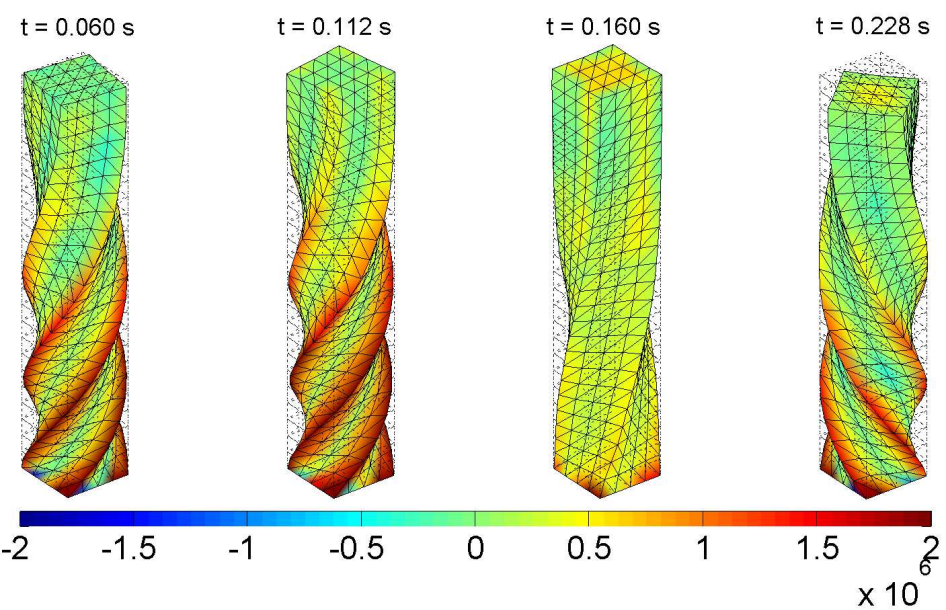

(b) Hu-Washizu mixed formulation

Figure 12: Twisting column: sequence of deformed shapes (pressure contour plot) using: (a) JST $\boldsymbol{p}$ - $\boldsymbol{F}$ FVM $(12 \times 12 \times 72 \times 6$ linear tetrahedral elements, $\alpha_{C F L}=0.3$ and $\left.\kappa^{(4)}=\frac{1}{128}\right)$; and (b) Hu-Washizu mixed formulation $(4 \times$ $4 \times 24 \times 6$ tetrahedral elements, $\rho_{\infty}=0.85$ and $\left.\Delta t=0.004 \mathrm{~s}\right)$. Results obtained with an initial sinusoidal rotational velocity $\Omega=100 \mathrm{rad} / \mathrm{s}$ (see (138)). A compressible Mooney-Rivlin material $\left(\alpha=\beta=\frac{\mu}{4}\right)$ is used such that Young's modulus $E=0.017 \mathrm{GPa}$, density $\rho_{0}=1.1 \mathrm{Mg} / \mathrm{m}^{3}$ and Poisson's ratio $\nu=0.3$. 


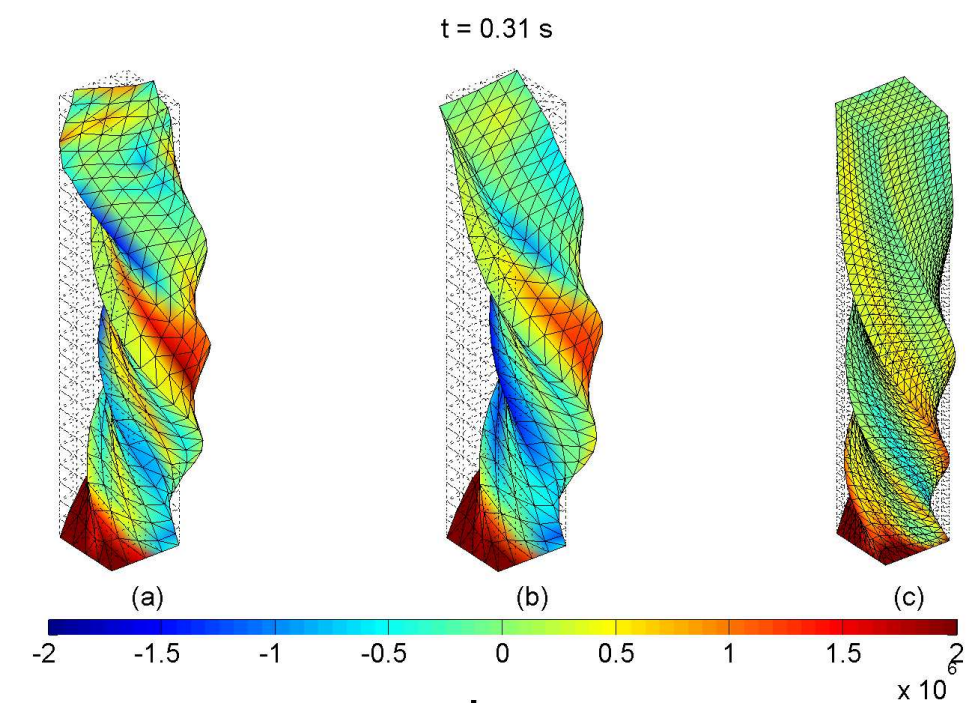

Figure 13: Twisting column: results obtained (e.g. hydrostatic pressure distribution) using the explicit stabilised $\boldsymbol{p}-\boldsymbol{F}-\boldsymbol{H}-J$ PG formulation with different values of time-integrated stabilisation at time $t=0.31$ s for $\boldsymbol{H}$ : (a) $\zeta_{\boldsymbol{H}}=0$ $\left(4 \times 4 \times 24 \times 6\right.$ linear tetrahedral elements); (b) $\zeta_{\boldsymbol{H}}=0.1(4 \times 4 \times 24 \times 6$ linear tetrahedral elements); and (c) $\zeta_{\boldsymbol{H}}=0.1(8 \times 8 \times 48 \times 6$ linear tetrahedral elements). Results obtained with an initial sinusoidal rotational velocity $\Omega=100 \mathrm{rad} / \mathrm{s}$ (see (138)). This example is run with Mooney-Rivlin model dominated by $\boldsymbol{H}$-term $\left(\alpha=0\right.$ and $\left.\beta=\frac{\mu}{2}\right)$ and material properties are such that Young's modulus $E=0.017 \mathrm{GPa}$, density $\rho_{0}=1.1 \mathrm{Mg} / \mathrm{m}^{3}$, Poisson's ratio $\nu=0.3$ and $\alpha_{C F L}=0.3$. Stabilising parameters: $\tau_{\boldsymbol{F}}=\tau_{\boldsymbol{H}}=\Delta t$, $\tau_{\boldsymbol{p}}=0.2 \Delta t, \zeta_{\boldsymbol{F}}=\tau_{J}=0$ and $\zeta_{J}=0.5 \frac{\mu}{\kappa}$. Lumped mass contribution. 


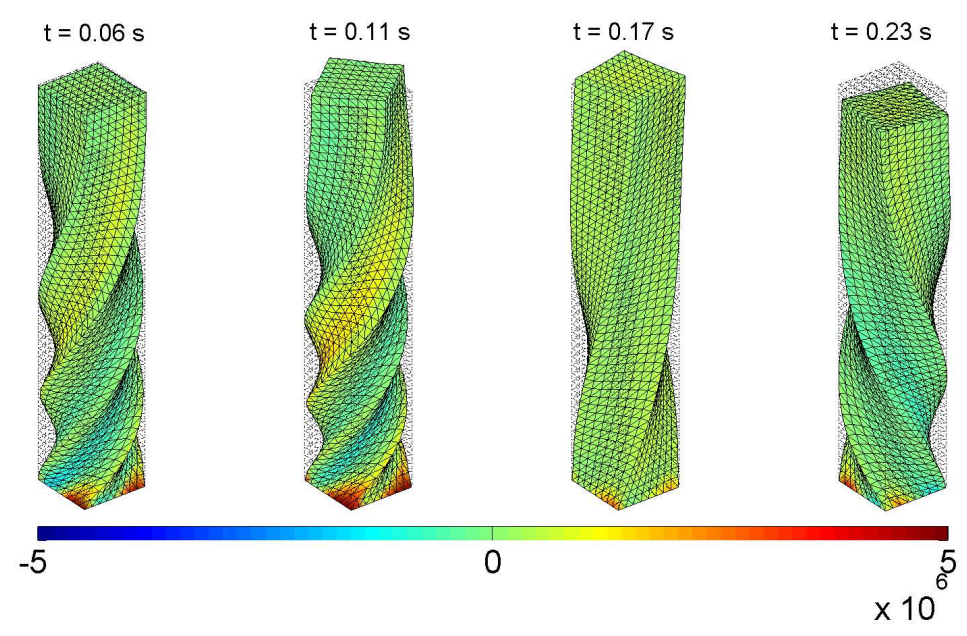

(a) Stabilised $\boldsymbol{p}$ - $\boldsymbol{F}$ - $\boldsymbol{H}$ - $J$ PG-FEM $\left(\tau_{\boldsymbol{F}}=\tau_{\boldsymbol{H}}=\Delta t, \tau_{\boldsymbol{p}}=0.2 \Delta t\right.$, $\left.\zeta_{\boldsymbol{H}}=0.1, \zeta_{J}=0.5 \frac{\mu}{\kappa}, \zeta_{\boldsymbol{F}}=\tau_{J}=0\right)$

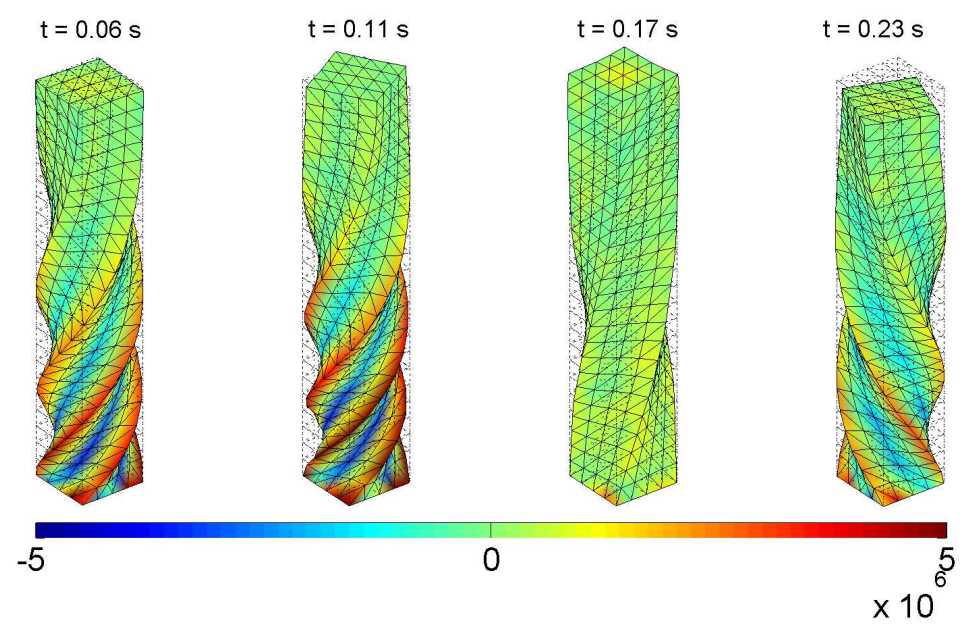

(b) Hu-Washizu mixed formulation

Figure 14: Twisting column: sequence of deformed shapes (hydrostatic pressure contour plot) using: (a) Stabilised $\boldsymbol{p}-\boldsymbol{F}-\boldsymbol{H}-J$ PG formulation $(8 \times 8 \times 48 \times 6$ linear tetrahedral elements, lumped mass contribution and $\alpha_{C F L}=0.3$ ); and (b) Hu-Washizu type variational mixed formulation $\left(4 \times 4 \times 24 \times 6\right.$ tetrahedral elements, $\rho_{\infty}=0.85$ and $\left.\Delta t=0.002 \mathrm{~s}\right)$. Results obtained with an initial sinusoidal rotational velocity $\Omega=100 \mathrm{rad} / \mathrm{s}$ (see (138)). This example is run with Mooney-Rivlin model $\left(\alpha=0\right.$ and $\left.\beta=\frac{\mu}{2}\right)$ and material properties are such that Young's modulus $E=0.017 \mathrm{GPa}$, density $\rho_{0}=1.1 \mathrm{Mg} / \mathrm{m}^{3}$ and Poisson's ratio $\nu=0.3$. 


\subsection{D tensile cube}

This example is an extension of the two dimensional tensile plate (see section 6.1) to a unit cube in three dimensions, to further illustrate the performance of the stabilised $\boldsymbol{p}-\boldsymbol{F}-\boldsymbol{H}-J$ mixed PG-FEM formulation. A unit cube clamped at the bottom and traction-free conditions on the rest of the boundaries is subjected to a sinusoidal variation in initial velocity field $\left.\boldsymbol{v}\right|_{t=0}=\left(0,0, v_{0} \sin \left(\frac{\Pi X_{3}}{2 L}\right)\right)^{T}\left(\right.$ where $\left.v_{0}=500 \mathrm{~m} / \mathrm{s}\right)$ which is compatible with the boundary. This problem is solved using a compressible Neo-Hookean material $\left(\alpha=\frac{\mu}{2}\right.$ and $\left.\beta=0\right)$ with Young's modulus $E=21 \mathrm{GPa}$, density $\rho_{0}=7 \mathrm{Mg} / \mathrm{m}^{3}$ and Poisson's ratio $\nu=0.3$. References $[1,8]$ reported that existing stabilised $\boldsymbol{p}-\boldsymbol{F}$ and $\boldsymbol{p}$ - $\boldsymbol{F}-J$ PG-FEM formulations perform extremely well and, therefore can be treated as reference solutions (see Figure 15) for comparison purposes. Comparisons of a sequence of deformed states, benchmarked against already published numerical methodologies, are shown in Figure 15 and Figure 16, respectively. It can be clearly seen that the numerical solutions obtained from the proposed $\boldsymbol{p}-\boldsymbol{F}-\boldsymbol{H}-J$ PG-FEM formu-

lation match extremely well with existing methodologies, without showing any non-physical hydrostatic pressure instabilities. 

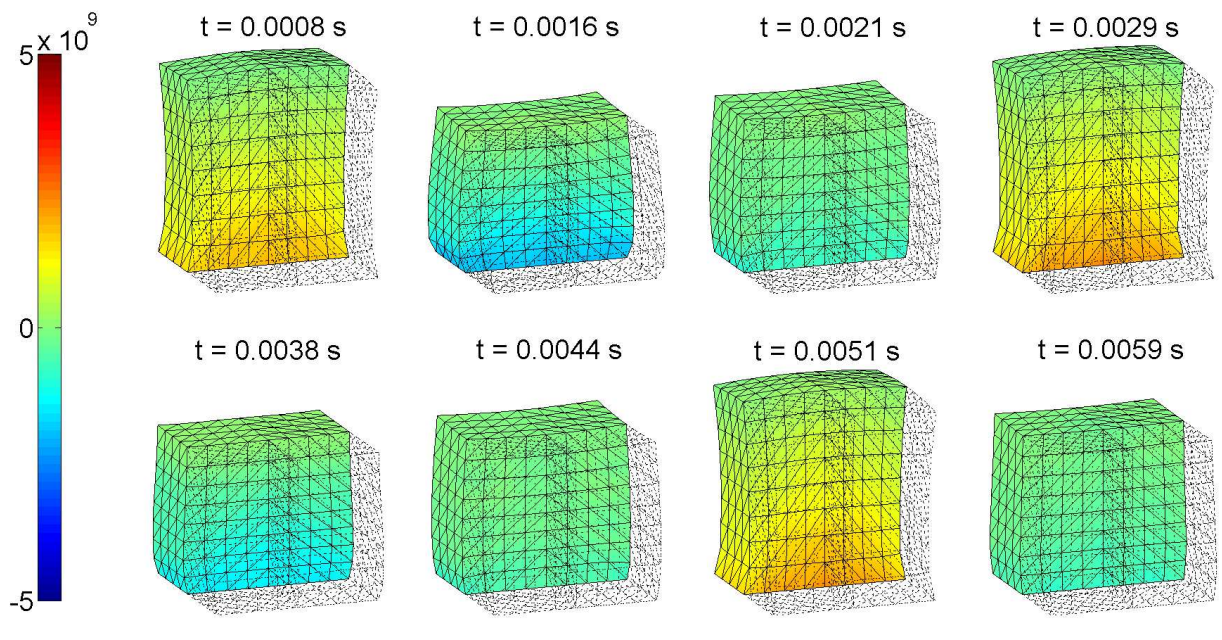

(a) Stabilised $\boldsymbol{p}$ - $\boldsymbol{F}$ PG-FEM [8]
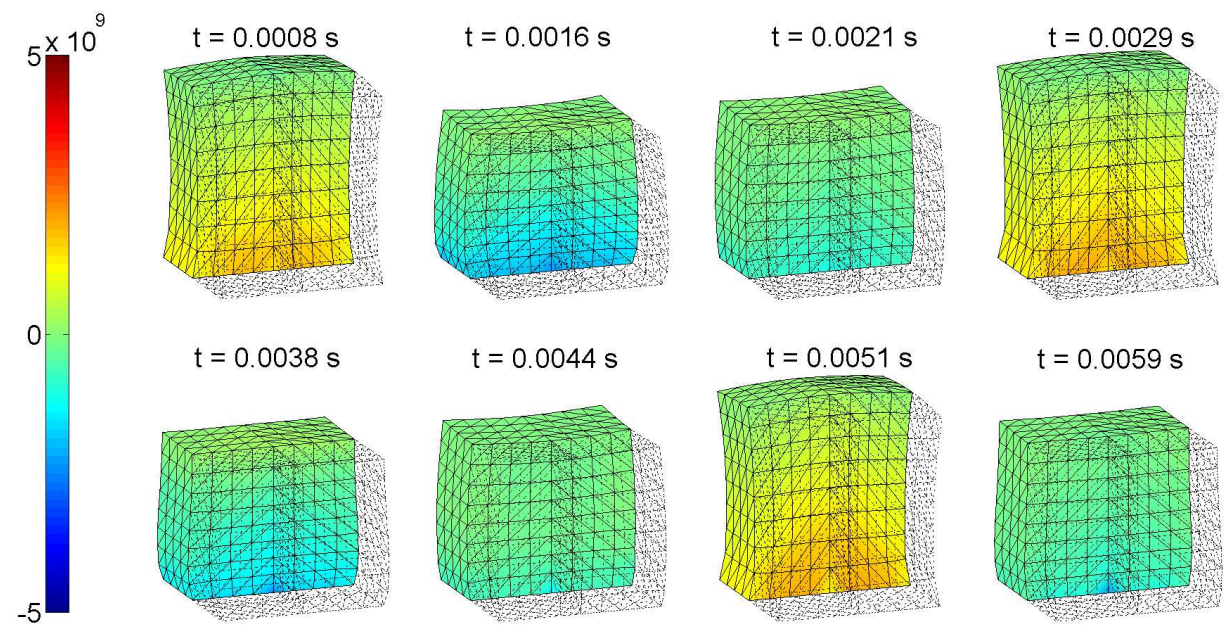

(b) Stabilised $\boldsymbol{p}$ - $\boldsymbol{F}$ - $J$ PG-FEM [1]

Figure 15: Tensile cube: a sequence of cross-sectional deformed shapes (pressure contour plot) using existing numerical methodologies: (a) $\boldsymbol{p}$ - $\boldsymbol{F}$ PG-FEM formulation $\left(\tau_{\boldsymbol{F}}=\Delta t, \zeta_{\boldsymbol{F}}=0.1, \tau_{\boldsymbol{p}}=0\right)$; and (b) $\boldsymbol{p}$ - $\boldsymbol{F}-J$ PG-FEM formulation $\left(\tau_{\boldsymbol{F}}=\Delta t, \tau_{\boldsymbol{p}}=0.2 \Delta t, \zeta_{J}=0.5 \frac{\mu}{\kappa}, \tau_{J}=\zeta_{\boldsymbol{F}}=0\right)$. Results obtained with a sinusoidal variation in initial velocity field $\left.\boldsymbol{v}\right|_{t=0}=\left[0,0, v_{0} \sin \left(\frac{\Pi X_{3}}{2 L}\right)\right]^{T}$ where $v_{0}=500 \mathrm{~m} / \mathrm{s}$. Compressible Neo-Hookean material $\left(\alpha=\frac{\mu}{2}\right.$ and $\left.\beta=0\right)$ is used such that Young's modulus $E=21 \mathrm{~Pa}$, density $\rho_{0}=7 \mathrm{Mg} / \mathrm{m}^{3}$, Poisson's ratio $\nu=0.3$ and $\alpha_{C F L}=0.3$. Discretisation of $8 \times 8 \times 8 \times 6$ linear tetrahedral elements. Lumped mass gontribution. 


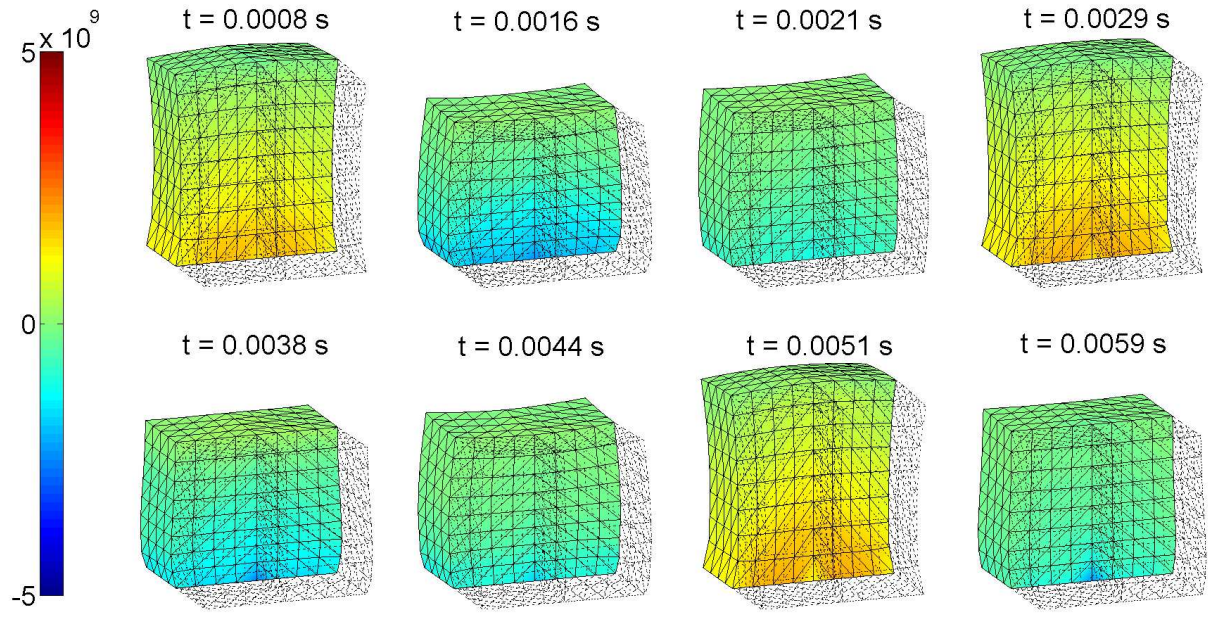

Figure 16: Tensile Cube: A sequence of cross-sectional deformed shapes (pressure contour plot) using stabilised $\boldsymbol{p}$ - $\boldsymbol{F}-\boldsymbol{H}$ - $J$ PG-FEM. Results obtained with a sinusoidal variation in initial velocity field $\left.\boldsymbol{v}\right|_{t=0}=\left[0,0, v_{0} \sin \left(\frac{\Pi X_{3}}{2 L}\right)\right]^{T}$ where $v_{0}=500 \mathrm{~m} / \mathrm{s}$. Compressible Neo-Hookean material $\left(\alpha=\frac{\mu}{2}\right.$ and $\left.\beta=0\right)$ is used such that Young's modulus $E=21 \mathrm{~Pa}$, density $\rho_{0}=7 \mathrm{Mg} / \mathrm{m}^{3}$, Poisson's ratio $\nu=0.3$ and $\alpha_{C F L}=0.3$. Discretisation of $8 \times 8 \times 8 \times 6$ linear tetrahedral elements. Stabilising parameters: $\tau_{\boldsymbol{F}}=\tau_{\boldsymbol{H}}=\Delta t, \tau_{\boldsymbol{p}}=0.2 \Delta t$, $\zeta_{\boldsymbol{F}}=\zeta_{\boldsymbol{H}}=\tau_{J}=0$ and $\zeta_{J}=0.5 \frac{\mu}{\kappa}$. Discretisation of $8 \times 8 \times 8 \times 6$ linear tetrahedral elements. Lumped mass contribution. 


\subsection{L-shaped block}

This benchmark problem, originally proposed by [105] and subsequently presented in $[1,52,105-107]$, is included in order to assess the ability of the algorithm to preserve the total angular momentum over a long term response. We consider the motion of a three-dimensional L-shaped block subjected to initial impulse traction boundary conditions at two of its sides described as follows (see Figure 17)

$$
\boldsymbol{F}_{1}(t)=-\boldsymbol{F}_{2}(t)=\left\{\begin{array}{lr}
t \boldsymbol{\eta}_{0}, & 0 \leq t<2.5 \\
(5-t) \boldsymbol{\eta}_{0}, & 2.5 \leq t<5 \\
\mathbf{0}, & t \geq 5
\end{array}\right.
$$

where $\boldsymbol{\eta}_{0}=(150,300,450)^{T}$. The material response is governed by the NeoHookean model $\left(\alpha=\frac{\mu}{2}\right.$ and $\beta=0$ ) where its physical properties are Young's modulus $E=50046 \mathrm{~Pa}$, density $\rho_{0}=1 \mathrm{Mg} / \mathrm{m}^{3}$ and Poisson's ratio $\nu=$ 0.3. A sequence of deformed states is illustrated in Figure 18 along with the contour plot of the module of the velocity distribution $\|\boldsymbol{v}\|$. As can be observed (refer to Figures 19), results are identical to those obtained using the alternative $\boldsymbol{p}$ - $\boldsymbol{F}$ and $\boldsymbol{p}$ - $\boldsymbol{F}$ - $J$ PG-FEM formulations $[1,8]$. In addition, Figure 20 presents the components of the angular momentum evolution calculated with the stabilised $\boldsymbol{p}-\boldsymbol{F}-\boldsymbol{H}-J$ PG formulation, where it can be seen that the angular momentum remains constant after removal of the external load.

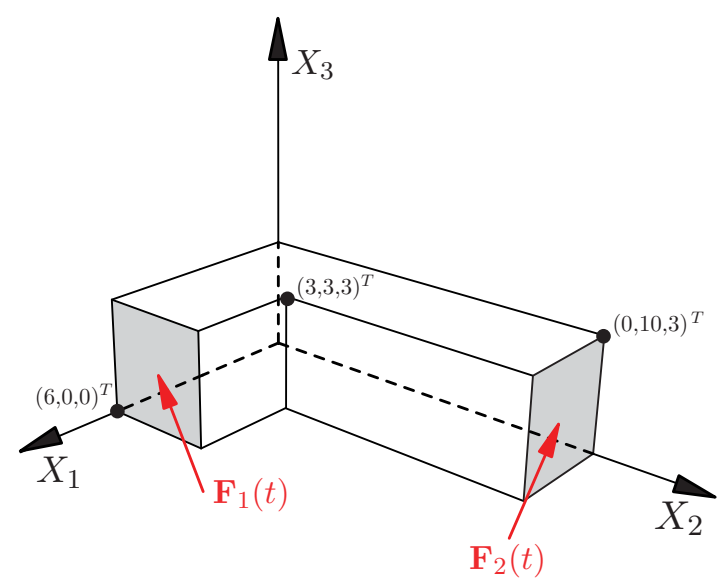

Figure 17: L-shaped block configuration 


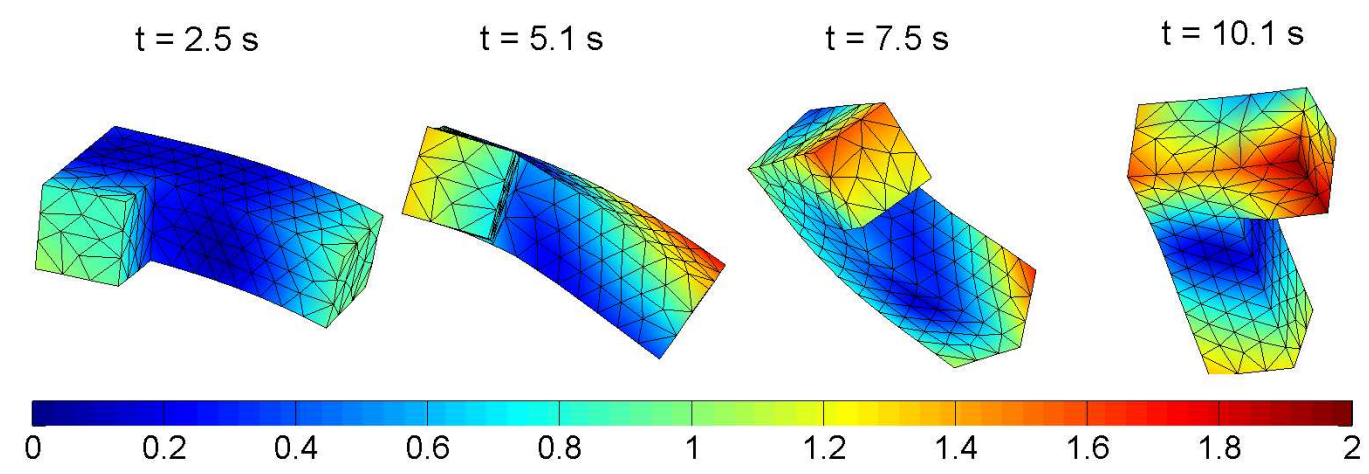

Figure 18: L-shaped block: results obtained (norm of the velocity distribution $\|\boldsymbol{v}\|)$ with an impulse traction boundary conditions (139) at two of its sides using the proposed $\boldsymbol{p}-\boldsymbol{F}-\boldsymbol{H}-J$ PG formulation. This example is run with a compressible Neo-Hookean constitutive model $\left(\alpha=\frac{\mu}{2}\right.$ and $\left.\beta=0\right)$ and material properties are Young's modulus $E=50046 \mathrm{~Pa}$, density $\rho_{0}=1$ $\mathrm{Mg} / \mathrm{m}^{3}$, Poisson's ratio $\nu=0.3$ and $\alpha_{C F L}=0.3$. Stabilising parameters: $\tau_{\boldsymbol{F}}=\tau_{\boldsymbol{H}}=\Delta t, \tau_{\boldsymbol{p}}=0.2 \Delta t, \zeta_{J}=0.5 \frac{\mu}{\kappa}, \tau_{J}=\zeta_{\boldsymbol{F}}=\zeta_{\boldsymbol{H}}=0$. Lumped mass contribution. 


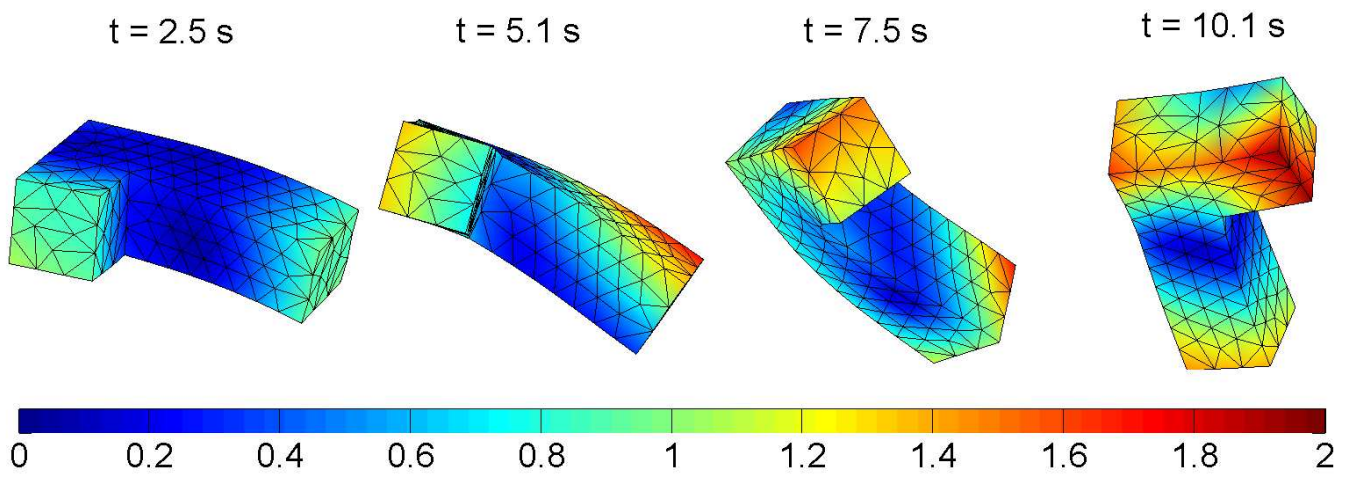

(a) Stabilised $\boldsymbol{p}-\boldsymbol{F}$ formulation $\left(\tau_{\boldsymbol{F}}=\Delta t, \tau_{\boldsymbol{p}}=0\right.$ and $\left.\zeta_{\boldsymbol{F}}=0.1\right)$

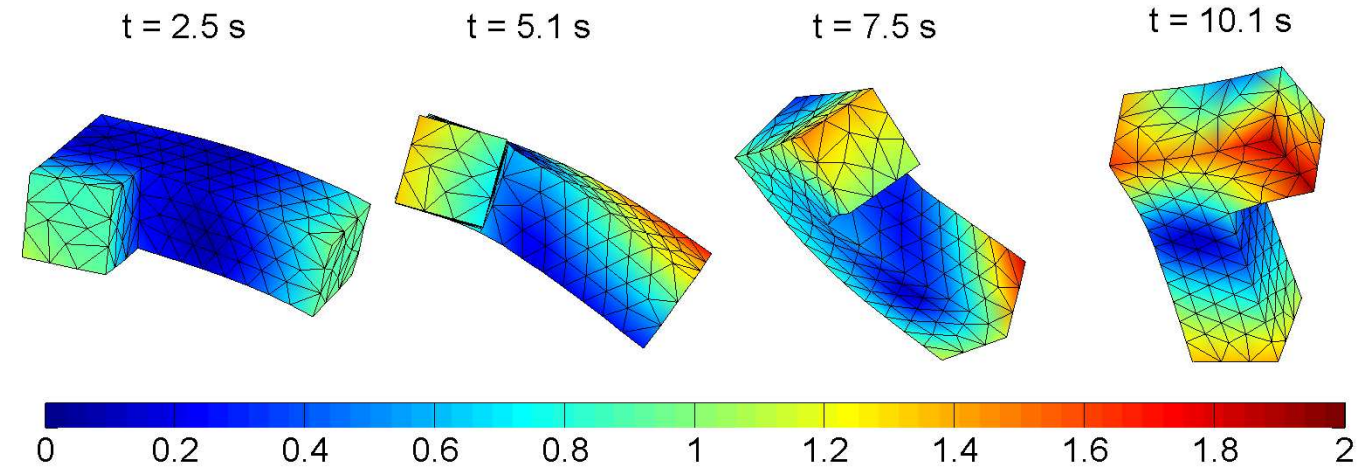

(b) Stabilised $\boldsymbol{p}-\boldsymbol{F}-J$ formulation $\left(\tau_{\boldsymbol{F}}=\Delta t, \tau_{\boldsymbol{p}}=0.2 \Delta t, \zeta_{J}=0.5 \frac{\mu}{\kappa}, \tau_{J}=\zeta_{\boldsymbol{F}}=0\right)$

Figure 19: L-shaped block: results obtained (norm of the velocity distribution $\|\boldsymbol{v}\|)$ with an impulse traction boundary conditions (139) at two of its sides using (a) Stabilised $\boldsymbol{p}$-F PG-FEM; and (b) Stabilised $\boldsymbol{p}$ - $\boldsymbol{F}$-J PG-FEM. This example is run with a compressible Neo-Hookean constitutive model ( $\alpha=\frac{\mu}{2}$ and $\beta=0$ ) and material properties are Young's modulus $E=50046 \mathrm{~Pa}$, density $\rho_{0}=1 \mathrm{Mg} / \mathrm{m}^{3}$, Poisson's ratio $\nu=0.3$ and $\alpha_{C F L}=0.3$. Lumped mass contribution. 


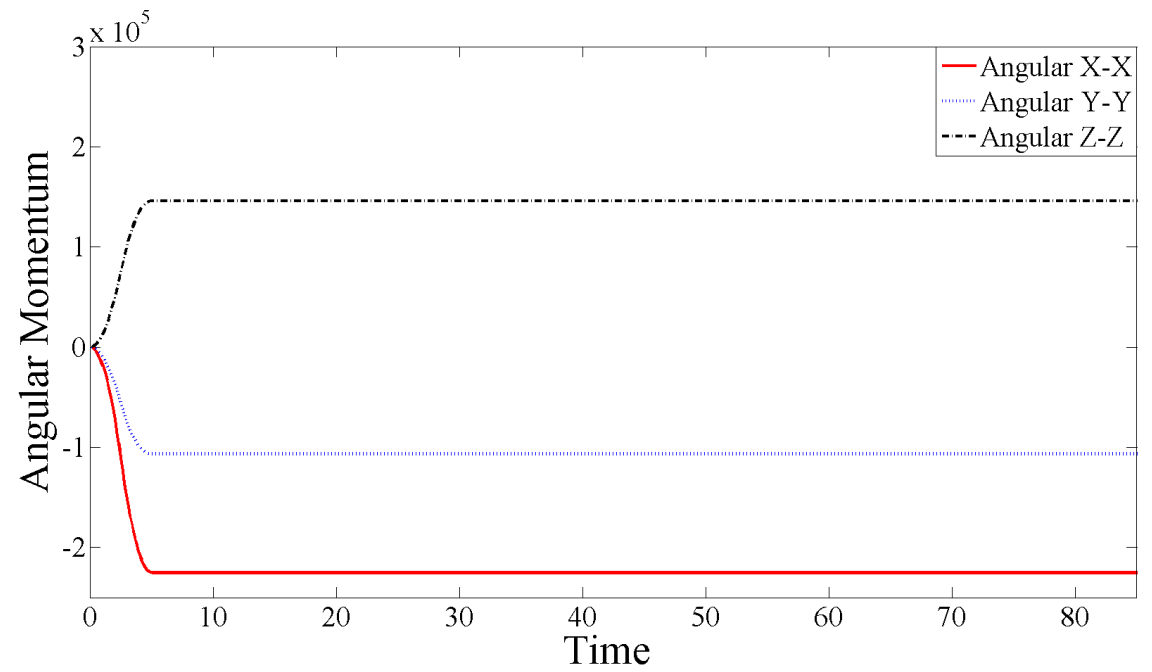

Figure 20: L-shaped block: results obtained with an impulse traction boundary conditions (139) at two of its sides using stabilised $\boldsymbol{p}-\boldsymbol{F}-\boldsymbol{H}-J$ PG formulation. This example is run with the Neo-Hookean constitutive model $\left(\alpha=\frac{\mu}{2}\right.$ and $\beta=0$ ) and material properties are such that Young's modulus $E=50046$ Pa, density $\rho_{0}=1 \mathrm{Mg} / \mathrm{m}^{3}$, Poisson's ratio $\nu=0.3$ and $\alpha_{C F L}=0.3$. Stabilising parameters: $\tau_{\boldsymbol{F}}=\tau_{\boldsymbol{H}}=\Delta t, \tau_{\boldsymbol{p}}=0.2 \Delta t, \zeta_{J}=0.5 \frac{\mu}{\kappa}, \tau_{J}=\zeta_{\boldsymbol{F}}=\zeta_{\boldsymbol{H}}=0$. Lumped mass contribution. 


\section{Concluding remarks}

In this paper, a new computational framework has been presented for the analysis of large strain fast solid dynamics in the case of isothermal elastic materials. In previous work published by the authors $[1,8,49,50,52]$, a mixed formulation was introduced where the conservation of linear momentum $\boldsymbol{p}$ is complemented with geometric conservation laws for the deformation gradient $\boldsymbol{F}$ and the Jacobian $J$ of the deformation, resulting in a first order system of hyperbolic equations. In this paper, the use of a new geometric conservation law for the co-factor $\boldsymbol{H}$ of the deformation has led to an enhanced mixed formulation $\boldsymbol{p}-\boldsymbol{F}-\boldsymbol{H}-J$.

The consideration of polyconvex [65] energy functionals has enabled the definition of generalised entropy functions and associated entropy fluxes [64] and the definition of suitable conjugate entropy variables $\boldsymbol{v}-\boldsymbol{\Sigma}_{\boldsymbol{F}^{-}} \boldsymbol{\Sigma}_{\boldsymbol{H}^{-}} \Sigma_{J}$ has enabled the introduction of a dual system of hyperbolic equations. Crucially, the new use of a tensor cross product $[46,61]$ greatly facilitates the algebraic manipulations of expressions involving the co-factor $\boldsymbol{H}$.

From the spatial discretisation point of view, the use of a stabilised Petrov-Galerkin framework [72] is presented in depth for both systems of hyperbolic equations, that is, when expressed in terms of either conservation or entropy variables. Finally, a comprehensive list of numerical examples in two and three dimensions has been presented, in order to benchmark the results obtained against alternative numerical strategies, including implicit and explicit time integrators as well as Finite Element and Finite Volume based discretisations.

In subsequent publications of this series, the authors will build upon the current work in order to explore three new aspects: first, the consideration of non-isothermal materials; second, a more sophisticated computational framework to ensure the exact satisfaction of the involutions [76] and, third, the formulation of the problem in alternative descriptions, namely: Updated Lagrangian, Eulerian and Arbitrary Lagrangian-Eulerian.

\section{Acknowledgements}

The first author acknowledges the financial support provided by the Sêr Cymru National Research Network for Advanced Engineering and Materials, United Kingdom. The second author would like to acknowledge the financial support received through "The Leverhulme Prize" awarded by The Leverhulme Trust, United Kingdom. The third author acknowledges the fi- 
nancial support provided by the EU funded project "Advanced Sustainable Manufacturing Technologies" (ASTUTE). The fourth author acknowledges the financial support of the EU FP7 framework, through the Initial Training Network "Advanced Techniques in Computational Mechanics", grant agreement 238548. The fifth author acknowledges the financial support provided by Swansea University, United Kingdom.

\section{Appendix A.}

One of the key elements of the framework proposed is the extension of the standard vector cross product to define the cross product between second order tensors and between second order tensors and vectors. This notation has already been introduced in reference [46, 61] but it is summarised here for completeness.

The cross product of a spatial vector $\boldsymbol{v}$ and a second order two-point tensor $\boldsymbol{A}$ to give a second order two-point tensor denoted $\boldsymbol{v} \times \boldsymbol{A}$ is defined so that when applied to a general material vector $\boldsymbol{V}$ gives:

$$
(\boldsymbol{v} \times \boldsymbol{A}) \boldsymbol{V}=\boldsymbol{v} \times(\boldsymbol{A} \boldsymbol{V}) ; \quad(\boldsymbol{v} \times \boldsymbol{A})_{i I}=\mathcal{E}_{i j k} v_{j} A_{k I} ; \quad i, I=1,2,3
$$

where $\mathcal{E}_{i j k}$ denotes the standard third order alternating tensor components, repeated indices indicate summation and $\times$ is the standard vector cross product. Similarly, the cross product of a two-point tensor $\boldsymbol{A}$ by a material vector $\boldsymbol{V}$ to give a second order two-point tensor denoted $\boldsymbol{A} \times \boldsymbol{V}$ is defined so that for every material vector $\boldsymbol{W}$ the following relationship applies:

$$
(\boldsymbol{A} \times \boldsymbol{V}) \boldsymbol{W}=\boldsymbol{A}(\boldsymbol{V} \times \boldsymbol{W}) ; \quad(\boldsymbol{A} \times \boldsymbol{V})_{i I}=\mathcal{E}_{I J K} A_{i J} V_{K}
$$

The cross tensor product of two two-point tensors $\boldsymbol{A}$ and $\boldsymbol{B}$ to give a new two-point tensor denoted $\boldsymbol{A} \times \boldsymbol{B}$ is defined so that for any arbitrary vectors $\boldsymbol{v}$ and $\boldsymbol{V}$ gives:

$$
\boldsymbol{v} \cdot(\boldsymbol{A} \times \boldsymbol{B}) \boldsymbol{V}=(\boldsymbol{v} \times \boldsymbol{A}):(\boldsymbol{B} \times \boldsymbol{V}) ; \quad(\boldsymbol{A} \times \boldsymbol{B})_{i I}=\mathcal{E}_{i j k} \mathcal{E}_{I J K} A_{j J} B_{k K} .
$$

Finally, the cross vector product of two two-point tensors to give a spatial vector is also defined by a cross product operation with respect to the first 
indices and a contraction with respect to the second set of indices, so that, ${ }^{8}$ $\boldsymbol{v} \cdot(\boldsymbol{A} \times \boldsymbol{B})=\boldsymbol{v} \cdot \mathcal{E}:\left(\boldsymbol{A} \boldsymbol{B}^{T}\right)=\operatorname{tr}\left(\boldsymbol{v} \times\left(\boldsymbol{A} \boldsymbol{B}^{T}\right)\right) ; \quad(\boldsymbol{A} \times \boldsymbol{B})_{i}=\mathcal{E}_{i j k} A_{j I} B_{k I}$.

The following list of properties are given in reference [46]. Note that $a$ is a scalar, $\boldsymbol{V}$ and $\boldsymbol{W}$ denote material vectors, $\boldsymbol{v}$ and $\boldsymbol{w}$ denote spatial vectors, $\boldsymbol{I}$ represents identity tensor with Kronecker delta components $[\boldsymbol{I}]_{i I}=\delta_{i I}$ and $\boldsymbol{A}, \boldsymbol{B}$ and $\boldsymbol{C}$ are second order two-point tensors:

$$
\begin{gathered}
\boldsymbol{A} \times \boldsymbol{B}=\boldsymbol{B} \times \boldsymbol{A} \\
\boldsymbol{A} \times \boldsymbol{B}=\boldsymbol{A}^{T} \times \boldsymbol{B}^{T} \\
\boldsymbol{A} \times(\boldsymbol{B}+\boldsymbol{C})=\boldsymbol{A} \times \boldsymbol{B}+\boldsymbol{A} \times \boldsymbol{C} \\
a(\boldsymbol{A} \times \boldsymbol{B})=(a \boldsymbol{A}) \times \boldsymbol{B}=\boldsymbol{A} \times(a \boldsymbol{B}) \\
(\boldsymbol{v} \otimes \boldsymbol{V})(\boldsymbol{w} \otimes \boldsymbol{W})=(\boldsymbol{v} \times \boldsymbol{w}) \otimes(\boldsymbol{V} \times \boldsymbol{W}) \\
\boldsymbol{v} \times(\boldsymbol{A} \times \boldsymbol{V})=(\boldsymbol{v} \times \boldsymbol{A}) \times \boldsymbol{V}=\boldsymbol{v} \times \boldsymbol{A} \times \boldsymbol{V} \\
\boldsymbol{A} \times(\boldsymbol{v} \otimes \boldsymbol{V})=-\boldsymbol{v} \times \boldsymbol{A} \times \boldsymbol{V} \\
(\boldsymbol{A} \times \boldsymbol{B}): \boldsymbol{C}=(\boldsymbol{B} \times \boldsymbol{C}): \boldsymbol{A}+(\boldsymbol{A} \times \boldsymbol{C}): \boldsymbol{B} \\
\boldsymbol{B} \times(\boldsymbol{A} \boldsymbol{V}) \times(\boldsymbol{B} \boldsymbol{W})+(\boldsymbol{B} \boldsymbol{V}) \times(\boldsymbol{A} \boldsymbol{W}) \\
\boldsymbol{A} \times \boldsymbol{I}=(\mathrm{tr} \boldsymbol{A}) \boldsymbol{I}-\boldsymbol{A}^{T} \\
\boldsymbol{I} \times \boldsymbol{I}=2 \boldsymbol{I} \\
(\boldsymbol{A} \times \boldsymbol{A}): \boldsymbol{A}=6 \operatorname{det} \boldsymbol{A} \\
\mathrm{Cof} \boldsymbol{A}=\frac{1}{2} \boldsymbol{A} \times \boldsymbol{A} \\
(\boldsymbol{A} \boldsymbol{C}) \times(\boldsymbol{B})=(\boldsymbol{A} \times \boldsymbol{B})(\mathrm{Cof} \boldsymbol{C})
\end{gathered}
$$

\footnotetext{
${ }^{8}$ Note that the symbol $\mathbf{x}$ is used when the result of the product is a tensor, whereas the symbol $\times$ is used when the outcome of the product is a vector.
} 


\section{Box 1. Enumeration of tensor cross products:}

$$
\begin{aligned}
{[\boldsymbol{v} \times \boldsymbol{A}]=} & {\left[\begin{array}{lll}
v_{y} A_{z X}-v_{z} A_{y X} & v_{y} A_{z Y}-v_{z} A_{y Y} & v_{y} A_{z Z}-v_{z} A_{y Z} \\
v_{z} A_{x X}-v_{x} A_{z X} & v_{z} A_{x Y}-v_{x} A_{z Y} & v_{z} A_{x Z}-v_{x} A_{z Z} \\
v_{x} A_{y X}-v_{y} A_{x X} & v_{x} A_{y Y}-v_{y} A_{x Y} & v_{x} A_{y Z}-v_{y} A_{x Z}
\end{array}\right] } \\
{[\boldsymbol{A} \times \boldsymbol{V}]=} & {\left[\begin{array}{lll}
A_{x Y} V_{Z}-A_{x Z} V_{Y} & A_{x Z} V_{X}-A_{x X} V_{Z} & A_{x X} V_{Y}-A_{x Y} V_{X} \\
A_{y Y} V_{Z}-A_{y Z} V_{Y} & A_{y Z} V_{X}-A_{y X} V_{Z} & A_{y X} V_{Y}-A_{y Y} V_{X} \\
A_{z Y} V_{Z}-A_{z Z} V_{Y} & A_{z Z} V_{X}-A_{z X} V_{Z} & A_{z X} V_{Y}-A_{z Y} V_{X}
\end{array}\right] } \\
{[\boldsymbol{A} \times \boldsymbol{B}]=} & {\left[\begin{array}{lll}
{[\boldsymbol{A} \times \boldsymbol{B}]_{x X}} & {[\boldsymbol{A} \times \boldsymbol{B}]_{x Y}} & {[\boldsymbol{A} \times \boldsymbol{B}]_{x Z}} \\
{[\boldsymbol{A} \times \boldsymbol{B}]_{y X}} & {[\boldsymbol{A} \times \boldsymbol{B}]_{y Y}} & {[\boldsymbol{A} \times \boldsymbol{B}]_{y Z}} \\
{[\boldsymbol{A} \times \boldsymbol{B}]_{z X}} & {[\boldsymbol{A} \times \boldsymbol{B}]_{z Y}} & {[\boldsymbol{A} \times \boldsymbol{B}]_{z Z}}
\end{array}\right] }
\end{aligned}
$$

$[\boldsymbol{A} \times \boldsymbol{B}]_{x X}=A_{y Y} B_{z Z}-A_{y Z} B_{z Y}+A_{z Z} B_{y Y}-A_{z Y} B_{y Z}$

$[\boldsymbol{A} \times \boldsymbol{B}]_{x Y}=A_{y Z} B_{z X}-A_{y X} B_{z Z}+A_{z X} B_{y Z}-A_{z Z} B_{y X}$

$[\boldsymbol{A} \times \boldsymbol{B}]_{x Z}=A_{y X} B_{z Y}-A_{y Y} B_{z X}+A_{z Y} B_{y X}-A_{z X} B_{y Y}$

$[\boldsymbol{A} \times \boldsymbol{B}]_{y X}=A_{x Z} B_{z Y}-A_{x Y} B_{z Z}+A_{z Y} B_{x Z}-A_{z Z} B_{x Y}$

$[\boldsymbol{A} \times \boldsymbol{B}]_{y Y}=A_{z Z} B_{x X}-A_{z X} B_{x Z}+A_{x X} B_{z Z}-A_{x Z} B_{z X}$

$[\boldsymbol{A} \times \boldsymbol{B}]_{y Z}=A_{z X} B_{x Y}-A_{z Y} B_{x X}+A_{x Y} B_{z X}-A_{x X} B_{z Y}$

$[\boldsymbol{A} \times \boldsymbol{B}]_{z X}=A_{x Y} B_{y Z}-A_{x Z} B_{y Y}+A_{y Z} B_{x Y}-A_{y Y} B_{x Z}$

$[\boldsymbol{A} \times \boldsymbol{B}]_{z Y}=A_{x Z} B_{y X}-A_{x X} B_{y Z}+A_{y X} B_{x Z}-A_{y Z} B_{x X}$

$[\boldsymbol{A} \times \boldsymbol{B}]_{z Z}=A_{x X} B_{y Y}-A_{x Y} B_{y X}+A_{y Y} B_{x X}-A_{y X} B_{x Y}$

\section{References}

[1] A. J. Gil, C. H. Lee, J. Bonet, M. Aguirre, A stabilised Petrov-Galerkin formulation for linear tetrahedral elements in compressible, nearly incompressible and truly incompressible fast dynamics, Computer Methods in Applied Mechanics and Engineering 276 (2014) 659-690.

[2] D. J. Benson, Computational methods in Lagrangian and Eulerian hydrocodes, Computer Methods in Applied Mechanics and Engineering 99 (1992) 235-394. 
[3] D. P. Flanagan, T. Belytschko, A uniform strain hexahedron and quadrilateral with orthogonal hourglass control, International Journal for Numerical Methods in Engineering 17 (1981) 679-706.

[4] G. L. Goudreau, J. O. Hallquist, Recent developments in large scale Lagrangian hydrocodes, Computer Methods in Applied Mechanics and Engineering 33 (1982) 725-757.

[5] N. P. Weatherill, O. Hassan, Efficient three-dimensional Delaunay triangulation with automatic point creation and imposed boundary constraints, International Journal for Numerical Methods in Engineering 37 (1994) 2005-2039.

[6] D. J. Payen, K. J. Bathe, A stress improvement procedure, Computers and Structures 112-113 (2012) 311-326.

[7] D. J. Payen, K. J. Bathe, Improved stresses for the 4-node tetrahedral element, Computers and Structures 89 (2011) 1265-1273.

[8] C. H. Lee, A. J. Gil, J. Bonet, Development of a stabilised PetrovGalerkin formulation for conservation laws in Lagrangian fast solid dynamics, Computer Methods in Applied Mechanics and Engineering 268 (2014) 40-64.

[9] C. R. Dohrmann, M. W. Heinstein, J. Jung, S. W. Key, W. R. Witkowski, Node-based uniform strain elements for three-node triangular and four-node tetrahedral meshes, International Journal for Numerical Methods in Engineering 47 (2000) 1549-1568.

[10] J. Bonet, H. Marriott, O. Hassan, An averaged nodal deformation gradient linear tetrahedral element for large strain explicit dynamic applications, Communications in Numerical Methods in Engineering 17 (2001) 551-561.

[11] T. Belytschko, W. K. Liu, B. Moran, Nonlinear finite elements for continua and structures, John Wiley and Sons, 2000.

[12] K. J. Bathe, Finite element procedures, Prentice Hall, 1996.

[13] M. W. Gee, C. R. Dohrmann, S. W. Key, W. A. Wall, A uniform nodal strain tetrahedron with isochoric stabilization, International Journal for Numerical Methods in Engineering 78 (2009) 429-443. 
[14] N. M. Newmark, A method of computation for structural dynamics, Journal of the Engineering Mechanics Division 85 (1959) 67-94.

[15] T. J. R. Hughes, The finite element method: Linear static and dynamic finite element analysis, Dover Publications, 2000.

[16] H. M. Hilber, T. J. R. Hughes, R. L. Taylor, Improved numerical dissipation for time integration algorithms in structural dynamics, Earthquake Engineering and Structural Dynamics 5 (1977) 283-292.

[17] W. L. Wood, M. Bossak, O. C. Zienkiewicz, An alpha modification of Newmark's method, International Journal for Numerical Methods in Engineering 15 (1980) 1562-1566.

[18] J. Chung, G. M. Hulbert, A time integration algorithm for structural dynamics with improved numerical dissipation: The generalized $\alpha$ method, Journal of Applied Mechanics 60 (1993) 371-375.

[19] D. D. Adams, W. L. Wood, Comparison of Hilber-Hughes-Taylor and Bossak $\alpha$ method for the numerical integration of vibration equations, International Journal for Numerical Methods in Engineering 19 (1983) $765-771$.

[20] A. J. Gil, P. D. Ledger, A coupled $h p$-finite element scheme for the solution of two-dimensional electrostrictive materials, International Journal for Numerical Methods in Engineering 91 (2012) 1158-1183.

[21] D. Jin, P. D. Ledger, A. J. Gil, An $h p$-fem framework for the simulation of electrostrictive and magnetostrictive materials, Computers and Structures 133 (2014) 131-148.

[22] T. Elguedj, Y. Bazilevs, V. M. Calo, T. J. R. Hughes, $\bar{B}$ and $\bar{F}$ projection methods for nearly incompressible linear and nonlinear elasticity and plasticity using higher-order NURBS elements, Computer Methods in Applied Mechanics and Engineering 197 (2008) 2732-2762.

[23] K. Washizu, Variational methods in elasticity and plasticity, Pergamon Press, Oxford, 1975.

[24] J. C. Nagtegaal, D. M. Park, J. R. Rice, On numerically accurate finite element solutions in the fully plastic range, Computer Methods in Applied Mechanics and Engineering 4 (1974) 153-177. 
[25] J. H. Argyris, P. C. Dunne, T. Angelopoulos, B. Bichat, Large natural strains and some special difficulties due to nonlinearity and incompressibility in finite elements, Computer Methods in Applied Mechanics and Engineering 4 (1974) 219-278.

[26] D. S. Malkus, T. J. R. Hughes, Mixed finite element methods - Reduced and selective integration techniques: A unification of concepts, Computer Methods in Applied Mechanics and Engineering 15 (1978) 63-81.

[27] T. J. R. Hughes, Generalization of selective integration procedures to anisotropic and nonlinear media, International Journal for Numerical Methods in Engineering 15 (1980) 1413-1418.

[28] J. C. Simo, R. L. Taylor, K. S. Pister, Variational and projection methods for the volume constraint in finite deformation elasto-plasticity, Computer Methods in Applied Mechanics and Engineering 51 (1985) $177-208$.

[29] E. A. de Souza Neto, D. Peric, M. Dutko, D. R. J. Owen, Design of simple low order finite elements for large strain analysis of nearly incompressible solids, International Journal of Solids and Structures 33 (1996) 3277-3296.

[30] T. Sussman, K. J. Bathe, A finite element formulation for nonlinear incompressible elastic and inelastic analysis, Computers and Structures 26 (1987) 357-409.

[31] B. Moran, M. Ortiz, C. F. Shih, Formulation of implicit finite element methods for multiplicative finite deformation plasticity, International Journal for Numerical Methods in Engineering 29 (1990) 483-514.

[32] J. C. Simo, F. Armero, Geometrically non-linear enhanced strain mixed methods and the method of incompatible modes, International Journal for Numerical Methods in Engineering 33 (1992) 1413-1449.

[33] M. A. Puso, A highly efficient enhanced assumed strain physically stabilized hexahedral element, International Journal for Numerical Methods in Engineering 49 (2000) 1029-1064. 
[34] J. Bonet, A. J. Burton, A simple average nodal pressure tetrahedral element for incompressible and nearly incompressible dynamic explicit applications, Communications in Numerical Methods in Engineering 14 (1998) 437-449.

[35] F. M. A. Pires, E. A. de Souza Neto, J. L. de la Cuesta Padilla, An assessment of the average nodal volume formulation for the analysis of nearly incompressible solids under finite strains, Communications in Numerical Methods in Engineering 20 (2004) 569-583.

[36] M. A. Puso, J. Solberg, A stabilized nodally integrated tetrahedral, International Journal for Numerical Methods in Engineering 67 (2006) 841-867.

[37] O. C. Zienkiewicz, J. Rojek, R. L. Taylor, M. Pastor, Triangles and tetrahedra in explicit dynamic codes for solids, International Journal for Numerical Methods in Engineering 43 (1998) 565-583.

[38] J. Bonet, H. Marriott, O. Hassan, Stability and comparison of different linear tetrahedral formulations for nearly incompressible explicit dynamic applications, International Journal for Numerical Methods in Engineering 50 (2001) 119-133.

[39] J. Bonet, P. Bhargava, A uniform deformation gradient hexahedron element with artificial hourglass control, International Journal for Numerical Methods in Engineering 38 (1995) 2809-2828.

[40] R. L. Taylor, A mixed-enhanced formulation for tetrahedral finite elements, International Journal for Numerical Methods in Engineering 47 (2000) 205-227.

[41] T. J. R. Hughes, L. P. Franca, M. Balestra, A new finite element formulation for computational fluid dynamics: V. Circumventing the Babuška-Brezzi condition: a stable Petrov-Galerkin formulation of the Stokes problem accommodating equal-order interpolations, Computer Methods in Applied Mechanics and Engineering 59 (1986) 85-99.

[42] W. Elmer, J. S. Chen, M. Puso, E. Taciroglu, A stable, meshfree, nodal integration method for nearly incompressible solids, Finite Elements in Analysis and Design 51 (2012) 81-85. 
[43] M. Cervera, M. Chiumenti, R. Codina, Mixed stabilized finite element methods in nonlinear solid mechanics: Part I: Formulation, Computer Methods in Applied Mechanics and Engineering 199 (2010) 2559-2570.

[44] F. Auricchio, L. B. da Veiga, C. Lovadina, A. Reali, R. L. Taylor, P. Wriggers, Approximation of incompressible large deformation elastic problems: Some unresolved issues, Computational Mechanics (2013) $1-15$.

[45] J. Schröder, P. Wriggers, D. Balzani, A new mixed finite element based on different approximations of the minors of deformation tensors, Computer Methods in Applied Mechanics and Engineering 200 (2011) 3583-3600.

[46] J. Bonet, A. J. Gil, R. Ortigosa, A computational framework for polyconvex large strain elasticity, Computer Methods in Applied Mechanics and Engineering (2014). Under review.

[47] S. K. Lahiri, J. Bonet, J. Peraire, A variationally consistent mesh adaptation method for triangular elements in explicit Lagrangian dynamics, International Journal for Numerical Methods in Engineering 82 (2010) 1073-1113.

[48] S. K. Lahiri, J. Bonet, J. Peraire, L. Casals, A variationally consistent fractional time-step integration method for incompressible and nearly incompressible Lagrangian dynamics, International Journal for Numerical Methods in Engineering 63 (2005) 1371-1395.

[49] I. A. Karim, C. H. Lee, A. J. Gil, J. Bonet, A two-step Taylor Galerkin formulation for fast dynamics, Engineering Computations 31 (2013) 366-387.

[50] C. H. Lee, A. J. Gil, J. Bonet, Development of a cell centred upwind finite volume algorithm for a new conservation law formulation in structural dynamics, Computers and Structures 118 (2013) 13-38.

[51] G. Kluth, B. Després, Discretization of hyperelasticity on unstructured mesh with a cell-centered Lagrangian scheme, Journal of Computational Physics 229 (2010) 9092-9118. 
[52] M. Aguirre, A. J. Gil, J. Bonet, A. A. Carreño, A vertex centred finite volume Jameson-Schmidt-Turkel (JST) algorithm for a mixed conservation formulation in solid dynamics, Journal of Computational Physics 259 (2014) 672-699.

[53] A. J. Gil, J. Bonet, J. Silla, O. Hassan, A Discrete Geometric Conservation Law (DGCL) for a cell vertex finite-volume algorithm on moving domains, International Journal for Numerical Methods in Biomedical Engineering 26 (2010) $770-779$.

[54] P. D. Thomas, C. K. Lombard, Geometric conservation law and its application to flow computations on moving grids, The American Institute of Aeronautics and Astronautics (AIAA) 10 (1979) 1030 - 1037.

[55] N. C. Nguyen, J. Peraire, Hybridizable Discontinuous Galerkin methods for partial differential equations in continuum mechanics, Journal of Computational Physics 231 (2012) 5955-5988.

[56] M. Aguirre, A. J. Gil, J. Bonet, C. H. Lee, An upwind vertex centred finite volume solver for Lagrangian solid dynamics, Journal of Computational Physics (2014). Under review.

[57] R. Codina, Pressure stability in fractional step finite element methods for incompressible flows, Journal of Computational Physics 170 (2001) $112-140$.

[58] A. J. Chorin, The numerical solution of the Navier-Stokes equations for an incompressible fluid, Bulletin of the American Mathematical Society 73 (1967) 928-931.

[59] A. J. Chorin, Numerical solution of the Navier-Stokes equations, Mathematics of Computation 22 (1968) 745-762.

[60] A. J. Chorin, On the convergence of discrete aapproximations to the Navier-Stokes equations, Mathematics of Computation 23 (1969) 341353.

[61] R. de Boer, Vektor- und Tensorrechnung für Ingenieure, SpringerVerlag, 1982. 
[62] D. H. Wagner, Symmetric-hyperbolic equations of motion for a hyperelastic material, Journal of Hyperbolic Differential Equations 06 (2009) 615-630.

[63] S. R. Wu, W. Qiu, Nonlinear transient dynamic analysis by explicit finite element with iterative consistent mass matrix, Communications in Numerical Methods in Engineering 25 (2009) 201-217.

[64] T. J. R. Hughes, L. P. Franca, M. Mallet, A new finite element formulation for computational fluid dynamics: I. Symmetric forms of the compressible Euler and Navier-Stokes equations and the second law of thermodynamics, Computer Methods in Applied Mechanics and Engineering 54 (1986) 223-234.

[65] J. M. Ball, Convexity conditions and existence theorems in nonlinear elasticity, Archive for Rational Mechanics and Analysis 63 (1976) 337403.

[66] J. M. Ball, Energy-minimising configurations in nonlinear elasticity, Archive for Rational Mechanics and Analysis 63 (1976) 337-403.

[67] J. M. Ball, Geometry, Mechanics and Dynamics, Springer, pp. 3-59.

[68] J. M. Ball, F. Murat, W quasiconvexity and variational problems for multiple integrals, Archive for Rational Mechanics and Analysis 63 (1976) 337-403.

[69] K. Zhang, A construction of quasiconvex functions with linear growth at infinity, Annali della Scuola Normale Superiore di Pisa, Classe di Scienze 97 (1992) 313-326.

[70] B. Dacorogna, Direct Methods in the Calculus of Variations, Springer, 2008.

[71] P. Ciarlet, Existence theorems in intrinsic nonlinear elasticity, Journal des mathématiques pures et appliqués 94 (2010) 229-243.

[72] T. J. R. Hughes, M. Mallet, A new finite element formulation for computational fluid dynamics: III. The generalized streamline operator for multidimensional advective-diffusive systems, Computer Methods in Applied Mechanics and Engineering 58 (1986) 305-328. 
[73] G. Scovazzi, Lagrangian shock hydrodynamics on tetrahedral meshes: A stable and accurate variational multiscale approach, Journal of Computational Physics 231 (2012) 8029-8069.

[74] J. Bonet, R. D. Wood, Nonlinear continuum mechanics for finite element analysis, Cambridge University Press, second edition, 2008.

[75] C. M. Dafermos, Quasilinear hyperbolic systems with involutions, Archive for Rational Mechanics and Analysis 94 (1986) 373-389.

[76] T. Barth, On the role of involutions in the Discontinuous Galerkin discretization of Maxwell and Magnetohydrodynamic systems, Springer New York 142 (2006) 69-88.

[77] G. H. Miller, An iterative Riemann solver for systems of hyperbolic conservation laws, with application to hyperelastic solid mechanics, Journal of Computational Physics 193 (2003) 198-225.

[78] R. Abedi, R. B. Haber, Riemann solutions and spacetime discontinuous Galerkin method for linear elastodynamic contact, Computer Methods in Applied Mechanics and Engineering 270 (2014) 150-177.

[79] J. E. Marsden, T. J. R. Hughes, Mathematical foundations of elasticity, Dover Publications, 1994.

[80] G. A. Holzapfel, Nonlinear solid mechanics: A continuum approach for engineering, Wiley and Sons, 2000.

[81] O. C. Zienkiewicz, R. L. Taylor, J. Z. Zhu, The finite element method : its basis \& fundamentals, volume 1, Butterworth-Heinemann, 6 edition, 2007.

[82] G. Scovazzi, Stabilized shock hydrodynamics: II. Design and physical interpretation of the SUPG operator for Lagrangian computations, Computer Methods in Applied Mechanics and Engineering 196 (2007) 967-978.

[83] G. Scovazzi, M. A. Christon, T. J. R. Hughes, J. N. Shadid, Stabilized shock hydrodynamics: I. A Lagrangian method, Computer Methods in Applied Mechanics and Engineering 196 (2007) 923-966. 
[84] K. Morgan, J. Peraire, Unstructured grid finite-element methods for fluid mechanics, Reports on Progress in Physics 61 (1998) 569-638.

[85] G. Scovazzi, A discourse on Galilean invariance, SUPG stabilization, and the variational multiscale framework, Computer Methods in Applied Mechanics and Engineering 196 (2007) 1108-1132.

[86] G. Scovazzi, Galilean invariance and stabilized methods for compressible flows, International Journal for Numerical Methods in Fluids 54 (2007) $757-778$.

[87] G. Scovazzi, E. Love, A generalized view on Galilean invariance in stabilized compressible flow computations, International Journal for Numerical Methods in Fluids 64 (2010) 1065-1083.

[88] G. Scovazzi, E. Love, M. J. Shashkov, A multi-scale Q1/P0 approach to Lagrangian shock hydrodynamics, Computer Methods in Applied Mechanics and Engineering 197 (2008) 1056-1079.

[89] G. Scovazzi, J. N. Shadid, E. Love, W. J. Rider, A conservative nodal variational multiscale method for Lagrangian shock hydrodynamics, Computer Methods in Applied Mechanics and Engineering 199 (2010) 3059-3100.

[90] F. Shakib, T. J. R. Hughes, Z. Johan, A new finite element formulation for computational fluid dynamics: X. The compressible Euler and Navier-Stokes equations, Computer Methods in Applied Mechanics and Engineering 89 (1991) 141-219.

[91] T. J. R. Hughes, G. Scovazzi, T. E. Tezduyar, Stabilized methods for compressible flows, Journal of Scientific Computing 43 (2010) 343-368.

[92] T. J. R. Hughes, G. Scovazzi, L. P. Franca, in: E. Stein, R. de Borst, T. J. R. Hughes (Eds.), Encyclopedia of Computational Mechanics, John Wiley and Sons, 2004.

[93] T. J. R. Hughes, Multiscale phenomena: Green's functions, the Dirichlet-to-Neumann formulation, subgrid scale models, bubbles and the origins of stabilized methods, Computer Methods in Applied Mechanics and Engineering 127 (1995) 387-401. 
[94] T. E. Tezduyar, M. Senga, Stabilization and shock-capturing parameters in SUPG formulation of compressible flows, Computer Methods in Applied Mechanics and Engineering 195 (2006) 1621-1632.

[95] T. E. Tezduyar, M. Senga, SUPG finite element computation of inviscid supersonic flows with $Y Z \beta$ shock-capturing, Computers and Fluids 36 (2007) 147-159.

[96] G. H. Miller, P. Colella, A high-order Eulerian Godunov method for elastic-plastic flow in solids, Journal of Computational Physics 167 (2001) 131-176.

[97] C. W. Shu, Efficient implementation of essentially non-oscillatory shock capturing schemes, Journal of Computational Physics 77 (1988) 439471.

[98] B. Cockburn, C. W. Shu, The Runge-Kutta discontinuous Galerkin finite element method for conservation laws V: Multidimensional systems, Journal of Computational Physics 141 (1998) 199-224.

[99] T. S. Zhang, A. Shahrouz, A slope limiting procedure in discontinuous Galerkin finite element method for gas dynamics applications, International Journal of Numerical Analysis and Modeling 2 (2005) 163-178.

[100] R. Courant, K. Friedrichs, H. Lewy, On the partial difference equations of mathematical physics, Mathematische Annalem 100 (1928) 32-74.

[101] A. N. Brooks, T. J. R. Hughes, Streamline upwind/Petrov-Galerkin formulations for convection dominated flows with particular emphasis on the incompressible Navier-Stokes equations, Computer Methods in Applied Mechanics and Engineering 32 (1982) 199-259.

[102] G. J. L. Beau, SUPG finite element computation of compressible flows with the entropy and conservation variable formulations, Computer Methods in Applied Mechanics and Engineering 104 (1993) 397 - 422.

[103] A. Soulaïmani, M. Fortin, Finite element solution of compressible viscous flows using conservative variables, Computer Methods in Applied Mechanics and Engineering 118 (1994) 319-350. 
[104] J. Donea, A. Huerta, Finite element methods for flow problems, Wiley and Sons, 2004.

[105] J. C. Simo, N. Tarnow, A new energy and momentum conserving algorithm for the non-linear dynamics of shells, International Journal for Numerical Methods in Engineering 37 (1994) 2527-2549.

[106] L. Noels, L. Stainier, J. P. Ponthot, An energy-momentum conserving algorithm for non-linear hypoelastic constitutive models, International Journal for Numerical Methods in Engineering 59 (2004) 84-114.

[107] X. N. Meng, T. A. Laursen, Energy consistent algorithms for dynamic finite deformation plasticity, Computer Methods in Applied Mechanics and Engineering 191 (2002) 1639-1675. 